Louisiana State University

LSU Digital Commons

Faculty Publications

Department of Biological Sciences

$1-1-2012$

\title{
The extrinsic proteins of Photosystem II
}

Terry M. Bricker

Louisiana State University

Johnna L. Roose

Louisiana State University

Robert D. Fagerlund

University of Otago

Laurie K. Frankel

Louisiana State University

Julian J. Eaton-Rye

University of Otago

Follow this and additional works at: https://digitalcommons.Isu.edu/biosci_pubs

\section{Recommended Citation}

Bricker, T., Roose, J., Fagerlund, R., Frankel, L., \& Eaton-Rye, J. (2012). The extrinsic proteins of Photosystem II. Biochimica et Biophysica Acta - Bioenergetics, 1817 (1), 121-142. https://doi.org/ 10.1016/j.bbabio.2011.07.006

This Article is brought to you for free and open access by the Department of Biological Sciences at LSU Digital Commons. It has been accepted for inclusion in Faculty Publications by an authorized administrator of LSU Digital Commons. For more information, please contact ir@lsu.edu. 
Review

\title{
The extrinsic proteins of Photosystem II ${ }^{\text {t }}$
}

\author{
Terry M. Bricker ${ }^{\mathrm{a}, *}$, Johnna L. Roose ${ }^{\mathrm{a}}$, Robert D. Fagerlund ${ }^{\mathrm{b}}$, Laurie K. Frankel ${ }^{\mathrm{a}}$, Julian J. Eaton-Rye ${ }^{\mathrm{b}}$ \\ a Department of Biological Sciences, Biochemistry and Molecular Biology Section, Louisiana State University, Baton Rouge, LA 70803, USA \\ ${ }^{\mathrm{b}}$ Department of Biochemistry, University of Otago, P.O. Box 56, Dunedin, New Zealand
}

\section{A R T I C L E I N F O}

\section{Article history:}

Received 11 May 2011

Received in revised form 11 July 2011

Accepted 12 July 2011

Available online 27 July 2011

\section{Keywords:}

PsbO

PsbP

PsbQ

PsbU

PsbV

\begin{abstract}
A B S T R A C T
In this review we examine the structure and function of the extrinsic proteins of Photosystem II. These proteins include PsbO, present in all oxygenic organisms, the PsbP and PsbQ proteins, which are found in higher plants and eukaryotic algae, and the PsbU, PsbV, CyanoQ and CyanoP proteins, which are found in the cyanobacteria. These proteins serve to optimize oxygen evolution at physiological calcium and chloride concentrations. They also shield the $\mathrm{Mn}_{4} \mathrm{CaO}_{5}$ cluster from exogenous reductants. Numerous biochemical, genetic and structural studies have been used to probe the structure and function of these proteins within the photosystem. We will discuss the most recent proposed functional roles for these components, their structures (as deduced from biochemical and X-ray crystallographic studies) and the locations of their proposed binding domains within the Photosystem II complex. This article is part of a Special Issue entitled: Photosystem II.
\end{abstract}

(c) 2011 Elsevier B.V. All rights reserved.

\section{Introduction}

Photosystem II (PSII) functions as a light energy-driven waterplastoquinone oxidoreductase. In higher plants, eukaryotic algae and cyanobacteria, light is trapped by and funneled through lightharvesting pigment-protein complexes (light-harvesting complex II or phycobilosomes) to the reaction center of PS II, which contains a specialized pigment complex, $\mathrm{P}_{680}$. After excitation, $\mathrm{P}_{680}$ becomes photooxidized and donates an electron to the primary acceptor of PS II, a protein-bound pheophytin. This charge separation is then further stabilized by the transfer of this electron, first to $Q_{A}$ and then to $Q_{B}$, protein-bound plastoquinones. The accumulation of two reducing equivalents on $\mathrm{Q}_{B}$ leads to its protonation and the formation of plastoquinol, which is then released from PS II. Concomitant to these electron transfers, $\mathrm{P}_{680}^{+}$is reduced by $\mathrm{Y}_{\mathrm{Z}}$, a tyrosyl residue $\left({ }^{161} \mathrm{Y}\right)$ located on the $\mathrm{D} 1$ protein. The subsequent reduction of $\mathrm{Y}_{\mathrm{Z}}$ leads to the accumulation of an oxidizing equivalent in the oxygen-evolving complex. The accumulation of four such oxidizing equivalents, in a manner consistent with the observed S-state transitions, leads to the release of oxygen from the complex (for reviews, see [1-4]). The oxygen-evolving complex contains a metal cluster consisting of four mixed valence manganese ions, a calcium ion, and five oxo ligands $\left(\mathrm{Mn}_{4} \mathrm{CaO}_{5}\right.$ cluster). Two chloride ions are also present flanking the cluster: $\mathrm{Cl}^{-} 1$ is $6.7 \AA$ from $\mathrm{Mn} 4$ and $\mathrm{Cl}^{-} 2$ is $7.4 \AA$ from $\mathrm{Mn} 2$ [5] in the $\mathrm{S}_{1}$ oxidation state. All of the protein ligands to this metal cluster are

\footnotetext{
ts This article is part of a Special Issue entitled: Photosystem II.

* Corresponding author at: Department of Biological Sciences Louisiana State University Baton Rouge, LA 70803, USA. Tel.: +1 225578 1555; fax: +1 2255782596. E-mail address: btbric@lsu.edu (T.M. Bricker).
}

provided by the D1 protein, with the singular exception that one manganese ligand is provided by the ${ }^{354} \mathrm{E}$ of CP43 [5-8].

In higher plants and cyanobacteria at least seven major intrinsic proteins appear to be absolutely required for oxygen evolution [9-11]. These are $\mathrm{CP} 47, \mathrm{CP} 43$, the D1 protein, the D2 protein, the $\alpha$ and $\beta$ subunits of cytochrome $b_{559}$, and the $4.8 \mathrm{kDa} p s b I$ gene product. Insertional inactivation of the genes for any of these components leads to a loss of normal PS II assembly and a loss of the ability to evolve oxygen. Additionally, a large number of low molecular mass intrinsic components are associated with PS II, although the functions of the majority of these proteins remain obscure, as their deletion only modestly affects PS II function. While PS II complexes containing only these components can evolve oxygen, they do so at very low rates (about 25\% of control) and require high, non-physiological levels of calcium and chloride [11]. In higher plants, three extrinsic proteins, PsbO, PsbP and PsbQ are required for maximal rates of oxygen evolution under physiological conditions. A fourth, possibly extrinsic protein, PsbR, is also present in higher plants and green algae. In cyanobacteria, the PsbO and PsbQ homologue (CyanoQ) proteins are present along with the PsbU and PsbV extrinsic components [12]. These latter two proteins are not present in higher plants. The cyanobacteria also contain a PsbP homologue (CyanoP); the stoichiometry of this component remains controversial $[13,14]$. Interestingly, while the green algae contain the same extrinsic components present in higher plants (PsbO, PsbP, PsbQ and PsbR), the red algae contain PsbO, PsbP, PsbQ' (a 20 kDa homologue of CyanoQ), PsbU and PsbV [15,16]; PsbR is not present in the red algae.

In this review, we will examine the structural and functional properties of these extrinsic protein components which function in support of oxygen evolution. We will principally focus on findings 

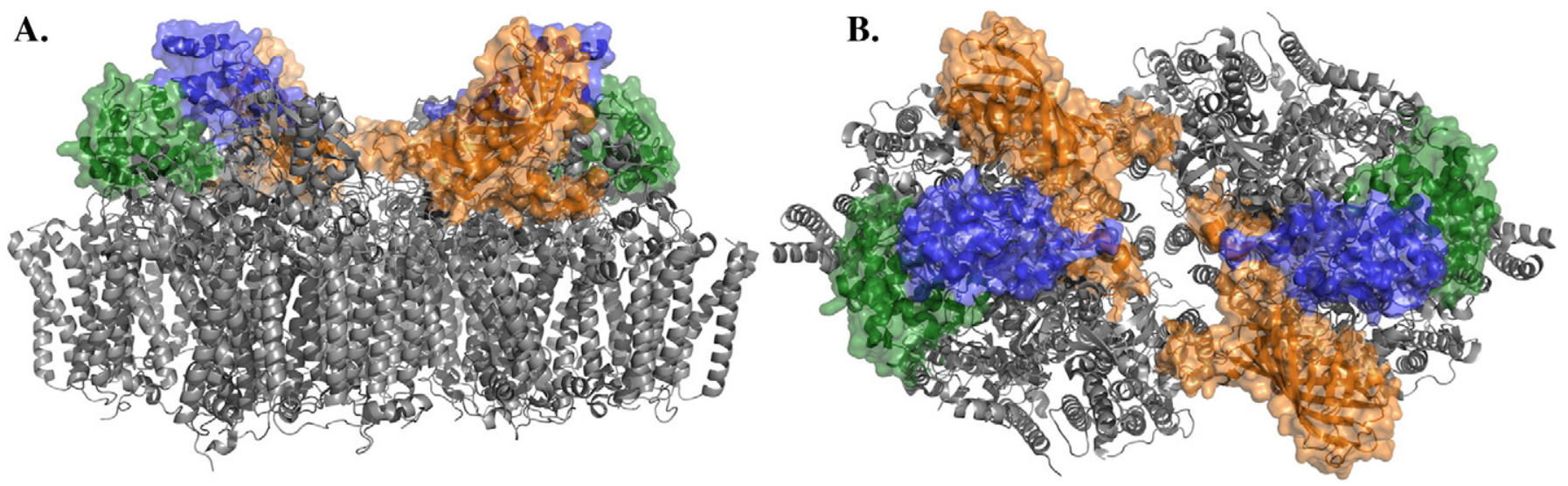

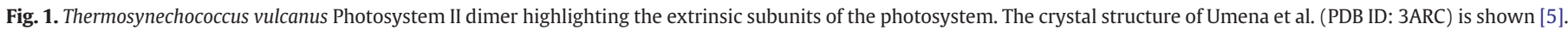

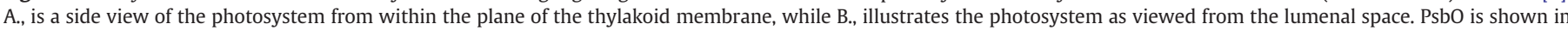

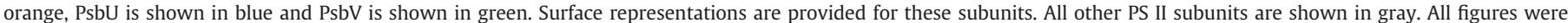
prepared in PyMol [259].

obtained during the last decade, which has witnessed an explosive growth in our understanding of the roles played by these proteins in the photosystem. Organizationally, we will first examine the PsbO, PsbP and PsbQ proteins. These components (or their homologues) are present in virtually all oxygenic organisms. The enigmatic PsbR protein, which is not present in cyanobacteria or red algae, will also be briefly examined. The cyanobacterial PsbU and PsbV proteins along with the cyanobacterial homologues of PsbQ (CyanoQ) and PsbP (CyanoP) will then be discussed. It should be noted here that we will not examine the extended PsbP and PsbQ families of proteins which are found in higher plants. The numerous members of these protein families may play other important roles within PS II, the Ndh dehydrogenase complex, and possibly other systems (for reviews, see [17-19]).

\subsection{Psbo structure}

Sequence alignments for the PsbO protein have been presented elsewhere [20,21] and indicate that PsbO is conserved across the higher plants, algal and cyanobacterial clades. Our understanding of the structural association of the PsbO protein within PS II comes principally from the crystal structures of the photosystem which have been obtained from the thermophilic cyanobacteria. These studies have, in large measure, confirmed earlier investigations which indicated that the PsbO component, in solution, exhibited a high degree of $\beta$-sheet secondary structure $[22,23]$ and that the protein was significantly elongated [24,25]. It should be noted that no crystal structures for the higher plant protein either as an isolated subunit or associated with the photosystem are currently available.

PsbO, in association with PS II from Thermosynechococcus vulcanus [5] (henceforth T. vulcanus), is shown in Fig. 1 in orange. This protein is located on the lumenal face of the complex and interacts with a variety of other PS II subunits including CP43, CP47, D1, D2 and PsbU. Since the PsbO protein can be removed from PS II membranes by treatment with $1 \mathrm{M} \mathrm{CaCl}_{2}$, it is hypothesized that charge-pair interactions provide the principal binding energy of PsbO to the photosystem. Within this context, it should be noted that PsbO remains firmly attached to PS II membranes even in the presence of $2 \mathrm{M} \mathrm{NaCl}$. The higher charge density of calcium (or other divalent cations) appears to be required to disrupt the interaction of PsbO with the PS II core components. The PsbO protein can also be removed from PS II membranes by treatment with $2.6 \mathrm{M}$ urea $+200 \mathrm{mM} \mathrm{NaCl}$ [9]. In this instance, the urea is hypothesized to weaken inter- or intramolecular hydrogen bond interactions sufficiently to allow release of the PsbO component by the $200 \mathrm{mM} \mathrm{NaCl}$.
To identify possible salt-bridge interactions of surfaces within the PS II crystal structure, we utilized the program KFC [26], which uses shape and residue properties to identify contact domains on multimeric proteins predicted to contribute the highest binding energy. We then examined the residues within the contact domains for possible chargepair interactions. Additionally, we used the program CAPTURE [27] to identify possibly relevant cation- $\pi$ interactions. The results from this analysis are shown in Table $1 .{ }^{1} \mathrm{CP} 43, \mathrm{CP} 47, \mathrm{D} 1$ and D2 all appear to possess residues which could contribute to the binding of PsbO.

Only a small subset of these residues, principally on the CP47 subunit, has been examined in more detail. Within the T. vulcanus crystal structure [5], a charge-pair "complex" appears to be formed involving the residues ${ }^{385} \mathrm{R}: \mathrm{CP} 47-{ }^{169} \mathrm{D}: \mathrm{PsbO}-{ }^{422} \mathrm{R}: \mathrm{CP} 47-{ }^{179} \mathrm{E}: \mathrm{PsbO}-{ }^{423} \mathrm{~K}$ : $\mathrm{CP} 47$ (Fig. 2). Please note that ${ }^{384} \mathrm{R}: \mathrm{CP} 47$ appears to interact with the C-terminal carboxyl group of the D2 protein $\left({ }^{352} \mathrm{~L}: \mathrm{D} 2\right)$. These residues are partially buried, which would increase the relative strength of the charge-pair interactions. The basic residue pairs of $C P 47,{ }^{384} R^{385} R$ and ${ }^{422} R^{423} R$, have been examined by site-directed mutagenesis in Synechocystis sp. PCC 6803 (henceforth Synechocystis 6803). Positive charges at these positions are absolutely conserved across all CP47 proteins. While alteration of the ${ }^{422} R^{423} R$ pair to neutral residues had a relatively small effect on oxygen evolution [28], modification of residues ${ }^{384} \mathrm{R}^{385} \mathrm{R}$ to either neutral $\left({ }^{384} \mathrm{G}^{385} \mathrm{G}\right)$ [29] or negatively charged $\left({ }^{384} \mathrm{E}^{385} \mathrm{E}\right)$ residues $[28]$ had a dramatic effect. Individual site-directed modification at either residue ${ }^{384} \mathrm{R}$ or ${ }^{385} \mathrm{R}$ also yielded mutants with significantly decreased oxygen evolution capability [28]. The double mutants evolved oxygen at rates comparable to that reported for the $\Delta p s b O$ mutant. Later studies, indicating that permeabilized thylakoids isolated from the $\mathrm{CP} 47{ }^{384} \mathrm{E}^{385} \mathrm{E}$ mutant bound the PsbO protein very weakly, ${ }^{2}$ and that the $\Delta p s b 0:{ }^{384} \mathrm{E}^{385} \mathrm{E}$ triple mutant exhibited a phenotype very similar to the $\Delta p s b O$ mutant, provided strong evidence that ${ }^{384} \mathrm{R}^{385} \mathrm{R}$ of $\mathrm{CP} 47$ provided an important binding site for PsbO to PS II [30]. It should be noted that detergent-permeabilized thylakoids isolated from the $\mathrm{CP} 47{ }^{384} \mathrm{G}^{385} \mathrm{G}$ mutant bound the PsbO protein significantly more strongly than the $\mathrm{CP} 47{ }^{384} \mathrm{E}^{385} \mathrm{E}$ mutant [30], indicating that other, yet unidentified residues also contribute to the association of PsbO with PS II. Neither of the PsbO residues $\left({ }^{169} \mathrm{D}\right.$ and ${ }^{179} \mathrm{E}$ ) predicted from the KFC analysis described above to participate

\footnotetext{
${ }^{1}$ The numbering of the specific amino acid residues in the various PS II proteins is problematic as various authors have used numbering derived from the immature translated gene sequence (with or without removal of the $\mathrm{N}$-terminal methionyl residue) or from the fully processed, mature protein, from a variety of different organisms. Except where indicated, we have used the numbering system from the $T$. vulcanus crystal structure of Umena et al. [5].

${ }^{2}$ His-tagged PS II particles containing mutations at residues ${ }^{384} \mathrm{R}^{385} \mathrm{R}$ of $\mathrm{CP} 47$ also lacked the PsbO subunit (C. Putnam-Evans and T. M. Bricker, unpublished).
} 
Table 1

Possible interacting residues of Thermosynechococcus vulcanus PsbO with other subunits of PS II. Binding domains were identified with KFC [26]. A putative cation- $\pi$ interaction was identified by the program CAPTURE. A possible inter-monomer interaction is shown in parentheses. The numbering scheme is for T. vulcanus as derived from the Umena et al. [5] crystal structure. It should be noted that these distances are slightly different for the two monomers. The closest distances are shown below.

\begin{tabular}{|c|c|c|c|}
\hline PsbO:CP 43 & PsbO:CP 47 & PsbO:D1 & PsbO:D2 \\
\hline \multicolumn{4}{|c|}{ Charge-pair interactions } \\
\hline${ }^{8} \mathrm{D}:{ }^{362} \mathrm{R}, 3.05 \AA$ & ${ }^{169} \mathrm{D}:{ }^{385} \mathrm{R}, 4.88 \AA$ & ${ }^{69} \mathrm{~K}:{ }^{103} \mathrm{D}, 3.2 \AA$ & ${ }^{160} \mathrm{~K}:{ }^{302} \mathrm{E}, 3.41 \AA$ \\
\hline \multirow{4}{*}{${ }^{99} \mathrm{D}:{ }^{381} \mathrm{~K}, 2.45 \AA$} & ${ }^{169} \mathrm{D}:{ }^{422} \mathrm{R}, 2.70 \AA$ & ${ }^{73} \mathrm{R}:{ }^{104} \mathrm{E}, 3.39 \AA$ & ${ }^{228} \mathrm{H}:{ }^{310} \mathrm{E}, 3.19 \AA$ \\
\hline & ${ }^{179} \mathrm{E}:{ }^{422} \mathrm{R}, 4.27 \AA$ & & \\
\hline & ${ }^{179} \mathrm{E}:{ }^{423} \mathrm{~K}, 5.20 \AA$ & & \\
\hline & $\left({ }^{59} \mathrm{~K}:{ }^{307} \mathrm{E}, 4.21 \AA\right)$ & & \\
\hline \multicolumn{4}{|c|}{ Cation- $\pi$ interaction } \\
\hline${ }^{7} \mathrm{Y}:{ }^{370} \mathrm{R}, 4.67 \AA$ & & & \\
\hline
\end{tabular}

in these charge-pair interactions with CP47 has been examined by sitedirected mutagenesis.

PsbO also may participate in an inter-monomer interaction with CP47, possibly stabilizing the PS II dimer [20]. KFC analysis indicates that ${ }^{59} \mathrm{~K}$ of PsbO could form a putative charge-pair interaction with ${ }^{307} \mathrm{E}$ of CP47. Such an interaction might explain the loss of PS II dimers in $\Delta p s b 0$ mutants [31]. An alternative hypothesis, however, is that increased photodamage occurring in the absence of PsbO in the $\Delta p s b O$ mutant accelerates the rate of monomer formation as a prelude to the repair of the photosystem.

Two residues in $\mathrm{CP} 43\left({ }^{362} \mathrm{R}\right.$ and $\left.{ }^{381} \mathrm{~K}\right)$ are also predicted to participate in charge-pair interactions with PsbO (Table 1). The residues ${ }^{362} \mathrm{R}$ and ${ }^{381} \mathrm{~K}$ lie within the Synechocystis 6803 deletion mutants ${ }^{3}$ $\Delta$ F345/E354 and $\Delta$ L364/D370, respectively [32]. These mutants did not assemble functional PS II reaction centers. Since the observed phenotype was much more extreme than that observed in the $\Delta p s b 0$ mutant, the loss of assembly of the photosystem cannot be ascribed to the possible loss of Psbo binding alone. The severe phenotype may be the result of the removal of ten and seven residues, respectively in these two deletion strains. The effects of alteration of the individual CP43 residues ${ }^{362} \mathrm{R}$ and ${ }^{381} \mathrm{~K}$ have not been directly probed by site-directed mutagenesis. The putative ${ }^{8} \mathrm{D}:$ PsbO- ${ }^{362} \mathrm{R}: \mathrm{CP} 43$ interaction is particularly interesting, however. A variety of N-terminal modifications of higher plant PsbO have been shown to affect PsbO binding to the photosystem. Eaton-Rye and Murata [33], using spinach PS II membranes, demonstrated that proteolytic removal of the N-terminal 16-18 amino acid residues from PsbO abolished its ability to bind to PS II. Popelkova et al. [34] identified two N-terminal domains ${ }^{4}\left({ }^{7} \mathrm{TYD}^{10} \mathrm{E}\right.$ and $\left.{ }^{15} \mathrm{TYL}^{18} \mathrm{E}\right)$ in the spinach PsbO protein which the authors demonstrated were required to bind the two copies of the PsbO protein per PS II monomer (see below) in higher plants. One of these domains is missing at the N-terminus of the cyanobacterial protein. Sequence alignment of higher plant and cyanobacterial PsbO sequences [21] indicates that the cyanobacteria uniformly exhibit a nine-ten amino acid deletion at the N-terminus (the ${ }^{7} \mathrm{TYD}^{10} \mathrm{E}$ domain lies within this deletion). These results may explain a major discrepancy between site-directed mutagenesis studies performed in higher plants and cyanobacteria. In spinach, site-directed modification of ${ }^{9} \mathrm{D}$ to either ${ }^{9} \mathrm{~N}$ or ${ }^{9} \mathrm{~K}$ had neither an effect on the binding of the modified PsbO protein to PsbO-depleted PS II membranes nor the restoration of oxygen-evolving activity [35]. In Synechocystis 6803, however, modification of ${ }^{9} \mathrm{D}$ to ${ }^{9} \mathrm{~K}$ weakened the binding of PsbO to thylakoid membranes and resulted in a moderate loss of oxygenevolving activity [30]. The findings of Popelokova et al. [34] demonstrate that the ${ }^{9} \mathrm{D}$ residue is not equivalent in the higher plant and cyanobacterial systems; in spinach, this residue lies in the first of two

\footnotetext{
${ }^{3}$ Synechocystis 6803 numbering.

${ }^{4}$ Spinach numbering.
}

binding domains, while in cyanobacteria it lies in the sole binding domain present in these organisms, which is equivalent to the second binding domain found in higher plants (please note that the ${ }^{9} \mathrm{D}$ residue in Synechocystis 6803 is equivalent to the ${ }^{8} \mathrm{D}$ residue in the T. vulcanus crystal structure [5]). The putative interacting residue, ${ }^{362} \mathrm{R}: \mathrm{CP} 43$, has not been examined by site-directed mutagenesis.

Finally, a number of residues in the D1 and D2 proteins are predicted to interact via charge-pair interactions with PsbO (Table 1). No site-directed mutagenesis experiments examining the residues in D1 and D2 with respect to PsbO binding have been reported. ${ }^{302} \mathrm{E}$ of the D2 protein is of particular interest, however. Its putative binding partner is ${ }^{160} \mathrm{~K}$ of PsbO, the modification of which dramatically altered the binding properties of the mutated PsbO protein in Thermosynechococcus elongatus ${ }^{5}$ (henceforth, T. elongatus) [36]. Other residues in this vicinity have also been implicated in the functional association of PsbO with the photosystem. Examination of the crystal structure indicates that the backbone carbonyl of ${ }^{158} \mathrm{D}$ of PsbO may be hydrogenbound to ${ }^{334} \mathrm{R}$ of $\mathrm{D} 1(3.0 \AA)$. This latter residue was modified in Synechocystis 6803 in an attempt to determine its involvement in extrinsic protein binding [37]. While no evidence was found indicating this role, the resultant mutant did exhibit a variety of PS II defects including altered $\mathrm{S}_{2}$ state properties and altered oxygen release kinetics. Modification of ${ }^{158} \mathrm{D}$ of PsbO yielded a mutant ${ }^{6}$ (D159N) which exhibited a $30 \%$ loss of steady-state oxygen evolution and loss of photoautotrophy in a $\Delta p s b V$ background, neither of which could be directly attributed to loss of PsbO from the photosystem [38]. These authors hypothesized that ${ }^{158} \mathrm{D}$ formed an intramolecular charge-pair with ${ }^{162} \mathrm{R}$. Examination of the T. vulcanus crystal structure [5] indicates that this is plausible, as these residues are separated by $2.7 \AA$.

Many of the results discussed above apply directly only to cyanobacterial PS II. It must be noted that while the PS II crystal structures have provided important insights into the structure of the photosystem, some questions remain. None of the current structures contain the CyanoQ or the CyanoP subunits. The former is almost certainly a stoichiometric component of the photosystem [12,13], while the latter also may be present in stoichiometric quantities [14], although this is controversial $[13,39]$. The observation that loss of CyanoQ destabilizes the binding of PsbV [40] also raises questions concerning the overall integrity of the observed subunit composition in the cyanobacterial structures currently available.

A number of studies, obtained principally by biochemical examination of higher plant PS II, appear inconsistent with current cyanobacterial crystal structures. Given the absence of a higher plant crystal structure it is unclear if these differences are due to real differences between the cyanobacterial and higher plant photosystem, possible limitations in the current cyanobacterial structures (particularly with regard to subunit composition), or investigator error. Numerous crosslinking studies have indicated that in higher plants [35,41,42], green algae [43] and cyanobacteria [44] the PsbO protein can be crosslinked to CP47 using the 0-length crosslinker 1-ethyl-3-(3-dimethylaminopropyl) carbodiimide (EDC). EDC crosslinks amino groups which are interacting with carboxylates via charge-pair interactions [45] and which are exposed to the bulk solvent. Odom et al. [46] mapped the crosslinked domain between these two components and reported that residues within the Nterminal tryptic peptide ${ }^{1} \mathrm{E}-{ }^{76} \mathrm{~K}$ of PsbO were crosslinked to residues within the domain ${ }^{364} \mathrm{E}-{ }^{440} \mathrm{D}$ of $\mathrm{CP} 47$. Examination of the T. vulcanus[5] crystal structure indicates that the only possible crosslinking candidates for this interaction are ${ }^{405} \mathrm{E}$ of $\mathrm{CP} 47$ and the residue ${ }^{66} \mathrm{~K}$ of PsbO in spinach. The latter residue corresponds to ${ }^{59} \mathrm{~K}$ in T. vulcanus. Interestingly, this would be the result of inter-monomer crosslinking. However, these

\footnotetext{
${ }^{5}$ In the original paper, Motoki et al. [36] examined “....a thermophilic cyanobacteria, Synechococcus elongatus". This organism is now named Thermosynechococcus elongatus. Synechococcus elongatus is now the genus and species name used for Synechococcus sp. PCC 7942.

${ }^{6}$ Synechocystis 6803 numbering.
} 


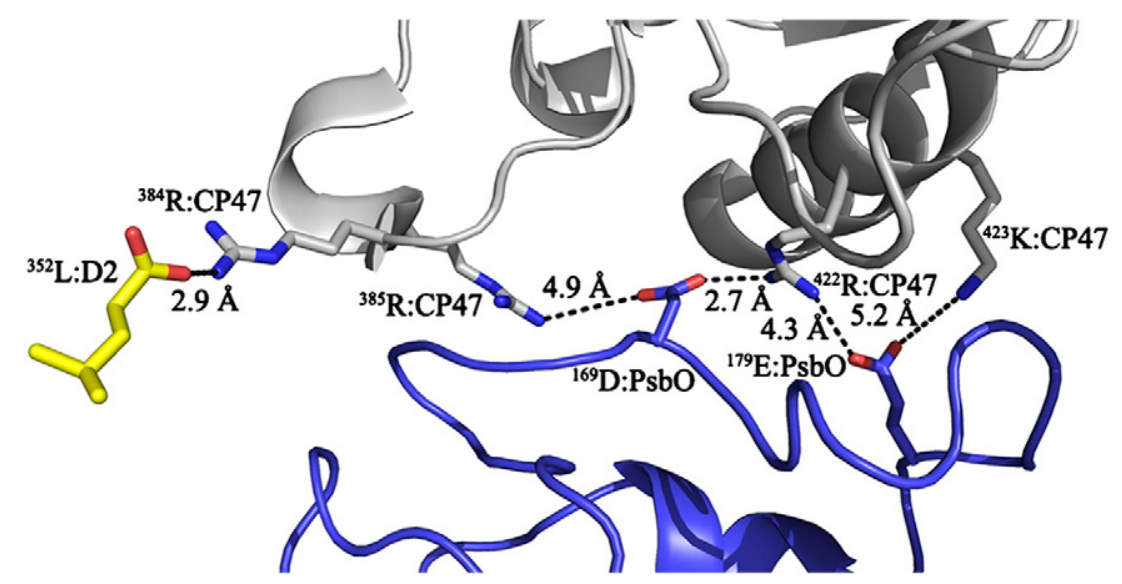

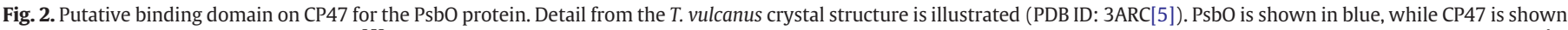

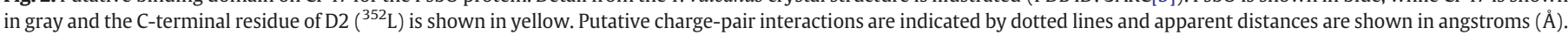

residues are separated by nearly $10 \AA$, thus precluding these residues from forming the charge-pair interaction required for EDC crosslinking.

Frankel and Bricker [47] reported that upon removal of the PsbO component from spinach PS II membranes, the domains ${ }^{304} \mathrm{~K}-{ }^{321} \mathrm{~K}$ and ${ }^{389} \mathrm{~K}-{ }^{419} \mathrm{~K}$ of $\mathrm{CP} 47$ became susceptible to modification with the amino group-reactive reagent NHS-biotin. Possible biotinylated residues include ${ }^{304} \mathrm{~K},{ }^{308} \mathrm{~K}$ and ${ }^{321} \mathrm{~K}$ in the first domain and ${ }^{389} \mathrm{~K},{ }^{418} \mathrm{~K}$ and ${ }^{419} \mathrm{~K}$ in the second. Four of these CP47 residues are conserved in T. vulcanus $\left({ }^{308} \mathrm{~K}\right.$, ${ }^{321} \mathrm{~K},{ }^{389} \mathrm{~K}$, and $\left.{ }^{418} \mathrm{~K}\right)$. None of these residues appears closely associated with the PsbO protein in the T. vulcanus structure. Interestingly, residues ${ }^{308} \mathrm{~K}$, ${ }^{389} \mathrm{~K}$, and possibly ${ }^{418} \mathrm{~K}$ are surface-exposed residues. If the structure of higher plant PS II is similar to that observed for cyanobacteria, these residues should have been labeled with NHS-biotin. No biotinylation, however, of any residues on CP47 was observed in the presence of PsbO in the higher plant system [47].

Enami et al. [48] used the crosslinker hexamethylene-diisocyanate (HMDI) to crosslink PS II components in spinach $\mathrm{NaCl}$-washed n-heptylthioglucoside-solubilized spinach PS II particles. HDMI can react with a variety of functional groups and can crosslink proteins which are within $11 \AA$ [49]. Individual crosslinked products were observed which contained both the PsbO protein and either PsbI or PsbE ( $\alpha$-subunit of cytochrome $b_{559}$ ). Examination of this result within the context of the T. vulcanus crystal structure yields interesting observations. The closest approach of PsbO with Psbl is 8-10 $\AA$, a distance which is consistent with the crosslinking result [48]. The nearest approach of PsbE with PsbO, however, is over $30 \AA$, a distance which cannot be spanned with HDMI. One possible explanation for this result could be inter-dimer crosslinking. However, this is unlikely since the experiment was performed on solubilized PS II particles and, consequently, the effective local PS II concentration would be much lower than would be observed in PS II membranes.

\section{Stoichiometry: cyanobacterial vs. higher plant}

In all cyanobacterial PS II crystal structures published to date, one copy of the PsbO protein is present per monomer. This has led many workers to assume that one copy of PsbO is present in all oxygenic organisms [50,51]. In higher plants, however, persistent biochemical observations indicate that two copies of PsbO are present per monomer of the photosystem. These studies have been reviewed extensively elsewhere $[21,52,53]$. Briefly, this evidence falls into three main categories: protein quantification experiments, rebinding studies and mutant characterization. Direct immuno-quantification using internal PsbO protein standards indicates that two copies of PsbO are present per monomer in both PS II membranes and $\beta$-Ddodecyl maltoside-solubilized PS II particles [54]. The number of PsbO copies bound is independent of the presence or absence of an intact
$\mathrm{Mn}_{4} \mathrm{CaO}_{5}$ cluster, although the $\mathrm{K}_{\mathrm{d}}$ for both PsbO copies is modulated by the intactness of the metal cluster [55]. Earlier, protein purification efforts had indicated that it was possible to isolate $1.7 \mathrm{~mol}$ of the PsbO component per mole PS II from PS II membranes; these membranes contain only strongly bound PsbO [56]. Rebinding studies from a number of laboratories examining higher plant PsbO binding to PS II membranes after extraction by $\mathrm{CaCl}_{2}$ or $\mathrm{NaCl}$-urea treatment demonstrated that binding of PsbO saturates at two copies of PsbO per PS II monomer [55,57-59]. The binding of native PsbO to PS II exhibits cooperativity with a Hill coefficient of 2.0 [55] and in some cases sigmoidal binding was evident [60]. Certain $\operatorname{mutated}^{7}[57,61]$ and chemically modified PsbO proteins [62] exhibit markedly sigmoidal binding isotherms. These are critical observations. The observed sigmoidicity necessitates cooperative binding which, by definition, requires at least two binding components. Finally, analysis of N-terminal truncation mutants has identified two binding domains, both of which must be present to observe normal PsbO binding stoichiometry $[34,63]$. One of these domains is not conserved in cyanobacterial sequences, perhaps explaining the apparent lack of a second copy of PsbO in these organisms. Functional studies indicate that the two copies of PsbO perform different functions within the photosystem. The first bound PsbO stabilizes the $\mathrm{Mn}_{4} \mathrm{CaO}_{5}$ and lowers the chloride requirement for oxygen evolution while the second PsbO further lowers the chloride requirement and optimizes oxygen evolution capacity [64,65].

If higher plants do contain two copies of the PsbO component, where is the second copy bound? At least two models are possible. First, the second copy could be bound symmetrically with respect to the first copy, on the lumenal surface of the PS II complex. This would necessitate the second copy associating principally with the CP47 protein in a similar manner as the first copy associates principally with CP43 in the cyanobacterial crystal structure. In this model, the second copy of PsbO would be quite distant from the $\mathrm{Mn}_{4} \mathrm{CaO}_{5}$ cluster. A second possibility is that the two PsbO copies form a homodimer. Three lines of circumstantial evidence support this hypothesis. First, during crystallization experiments of the spinach protein, only PsbO dimers are observed in the microcrystals which formed [66]. Second, single particle analysis of PS II supercomplexes has interpreted the density arising from the PsbO subunit as being aglobular and/or being present as a dimer [67]. Finally, re-examination of the chemical modification data presented by Frankel and Bricker [68] indicated that the majority of the PsbO residues which were labeled when PsbO was in solution but which were not labeled when PsbO was associated with $\mathrm{NaCl}$-washed PS II membranes are solvent-exposed in the

\footnotetext{
7 It should be noted that in the only reconstitution study performed with cyanobacterial mutated PsbO proteins, sigmoidal binding was also observed for some mutants [36].
} 
T. vulcanus crystal structure. These residues may define the interaction domain of the second copy of the PsbO protein with higher plant PS II. It should be noted that these models are not necessarily exclusive. A homodimer of PsbO would almost certainly also interact with the CP47 present in the other PS II monomer. If this were the case, it could explain a number of the discrepant results described above [46,47]. Clearly, resolution of this question of PsbO stoichiometry in higher plants ultimately will require crystal structures of intact higher plant PS II.

While the cyanobacterial and higher plant PsbO proteins are quite similar, some differences have been noted. In addition to the $\mathrm{N}$-terminal truncation which is present in all cyanobacteria (see above), several loop domains are also different. These features have been examined in detail elsewhere $[20,53]$. The most striking of these differences is the presence of a fourteen amino acid residue "cyanoloop" [20] which is present in all cyanobacteria. In T. vulcanus this loop extends from ${ }^{129} \mathrm{~T}-{ }^{142} \mathrm{~F}$ while the analogous loop in spinach is quite small $\left({ }^{136} \mathrm{G}-{ }^{141} \mathrm{~F}\right)$. The functional significance of this difference is unclear; however, it has been noted that the cyano-loop effectively compensates for the ubiquitous cyanobacterial N-terminal truncation, so that all PsbO proteins are approximately the same length. It has been suggested that this may have functional implications $[53,69]$.

\section{Psbo function}

Functionally, what parameters of the oxygen-evolving process are affected by the biochemical or genetic removal of PsbO? Numerous investigators have examined this question using higher plant PS II membranes which were depleted of the PsbO component by either $\mathrm{CaCl}_{2}$ [70] or NaCl-urea [9] treatment. These preparations, while completely lacking the PsbO protein, retain the $\mathrm{Mn}_{4} \mathrm{CaO}_{5}$ cluster and the ability to evolve oxygen, albeit at very low rates [11]. It should be noted that these studies were typically performed at high chloride and calcium concentrations to maintain the intactness of the $\mathrm{Mn}_{4} \mathrm{CaO}_{5}$ cluster (see below) and to compensate for the absence of the PsbP and PsbQ subunits which are also removed by these treatments. Ono and Inoue [71], using thermoluminescence as a probe for S-state transitions, observed that the $S_{3} \rightarrow\left[S_{4}\right] \rightarrow S_{0}$ transition was significantly slowed. Miyao et al. [72] performed flash oxygen yield measurements on PsbOdepleted PS II membranes. These studies indicated that the $\mathrm{S}_{2}$ lifetime of the PsbO-depleted samples increased five-fold and that the lifetime of the $S_{3}$ state also increased. Additionally, the $S_{2} \rightarrow S_{3}$ transition was slowed and the $S_{3} \rightarrow\left[S_{4}\right] \rightarrow S_{0}$ transition was retarded two- to threefold. Similar results have been obtained in vivo. An Arabidopsis thaliana (hereafter Arabidopsis) mutant which lacks a functional PsbO-1 protein $[59,73]$, the isoform responsible for normal PS II activity, exhibited longlived $S_{2}$ and $S_{3}$ state lifetimes [74]. Thermoluminescence experiments performed with an Arabidopsis T-DNA mutant which lacked the PsbO-1 protein also indicated an increased stability of the $S_{2}$ state, and EPR measurements indicated a lower yield of the $\mathrm{S}_{2}$ multiline signal [75]. The analysis of cyanobacterial $\triangle p s b O$ mutants has yielded very similar results. Flash oxygen yield measurements on the $\Delta p s b 0$ mutant of Synechocystis 6803 showed an increased stabilization of the $S_{2}$ and $S_{3}$ states and that the $S_{3} \rightarrow\left[S_{4}\right] \rightarrow S_{0}$ transition was slowed at least five-fold [76]. Thermoluminescence measurements on this strain also indicated a higher stability of the $S_{2}$ and $S_{3}$ states $[76,77]$ in this mutant. A parallel retardation in the rate of $\mathrm{Y}_{Z^{\bullet}}$ reduction was also observed [78] which indicated slower electron transfer from the $\mathrm{Mn}_{4} \mathrm{CaO}_{5}$ cluster to $\mathrm{Y}_{Z^{*}}$.

Considerable circumstantial evidence is available which indicates that the PsbO protein functions to modulate the chloride and calcium ${ }^{8}$ requirements for oxygen evolution. The oxygen evolution requirements for both of these ions are significantly lowered in the presence of the PsbO component [11]. The effects of chloride and/or calcium

\footnotetext{
${ }^{8}$ For excellent reviews on the role of chloride and calcium in PS II the reader should consult references $[143,144]$.
}

depletion on the oxygen evolution characteristics of higher plant PS II membranes are very similar qualitatively to the effects observed upon removal of the PsbO protein, as described above. This correlation is particularly strong with respect to chloride. Oxygen-evolving activity is markedly inhibited in chloride-depleted PS II membranes [79], with residual activities less than $20 \%$ of the control reported. Chloride depletion leads to a decrease in intensity of the $S_{2}$ multiline signal with a concomitant increase of the $g=4.0$ signal $[79,80]$. A similar decrease in the multiline signal was observed upon removal of the PsbO component [81]. Flash-induced UV absorbance changes indicated a twenty-fold stabilization of the $S_{2}$ state lifetime, a retardation of the $S_{2} \rightarrow S_{3}$ transition and the absence of any detectable $S_{3} \rightarrow\left[S_{4}\right] \rightarrow S_{0}$ transition upon chloride depletion [82]. No effect on $S_{3}$ lifetime, however, was observed. Proton release experiments indicated that $S-$ state transitions were blocked after $S_{2}$ [83]. Ono et al. [84] reported that the XANES edge shifts associated with S-state advancement were blocked at the $S_{2}$ state. Finally, chloride was demonstrated to be required for the $S_{2} \rightarrow S_{3}$ and $S_{3} \rightarrow\left[S_{4}\right] \rightarrow S_{0}$, but not for the $S_{0} \rightarrow S_{1}$ or the $S_{1} \rightarrow S_{2}$ transitions [82]. Depletion of calcium also prevents the $S_{2} \rightarrow S_{3}$ transition [85]; however, the $S_{1} \rightarrow S_{2}$ transition was also slowed $[86,87]$. These latter results may correlate to the observation that removal of the PsbO protein leads to a lower yield of the formation of the $S_{2}$-multiline signal [81]. Finally, thylakoids isolated from Arabidopsis mutants lacking the PsbO-1 protein exhibit longlived $S_{2}$ and $S_{3}$ states [88]; this phenotype can be chemically complemented by the addition of calcium and a calcium ionophore but not by the addition of chloride, alone. The mechanism(s) by which the PsbO protein could modulate the chloride and calcium requirements for oxygen evolution, however, remain unclear.

The PsbO component was earlier termed the 'manganese-stabilizing protein' based on the observation that removal of this component from higher plant PS II membranes leads to a destabilization of the $\mathrm{Mn}_{4} \mathrm{CaO}_{5}$ cluster at low chloride concentrations. In the absence of added chloride, two of the four manganese become paramagnetically uncoupled [89] and are eventually lost to the bulk media [90,91]. In the presence of high chloride concentrations ( $>100 \mathrm{mM}$ ) the $\mathrm{Mn}_{4} \mathrm{CaO}_{5}$ cluster remains intact and oxygen can be evolved even in the complete absence of the PsbO component $[11,90]$, although at significantly lower rates $(20-40 \%$ of control). It has recently been reported that intermediate chloride concentrations $(10-20 \mathrm{mM})$ preserve the integrity of the $\mathrm{Mn}_{4} \mathrm{CaO}_{5}$ cluster to varying degrees, and this is modulated in the higher plant system by the number of the PsbO subunits associated with each monomer [64]. While the PsbO protein stabilizes the active site $\mathrm{Mn}_{4} \mathrm{CaO}_{5}$ cluster, EXAFS experiments performed on PS II membranes depleted of PsbO indicated that essentially no change occurred in the structure of the metal cluster upon removal of PsbO [92]. This result strongly indicates that the PsbO protein does not provide any ligands directly to the $\mathrm{Mn}_{4} \mathrm{CaO}_{5}$ cluster. Early investigations also suggested that PsbO was directly involved in calcium binding at the oxygen-evolving site of PS II [93-95]; later studies indicated that PsbO was not involved in this process (see Seidler, [96], for an in-depth discussion). Seidler and Rutherford [97], for instance, demonstrated that in the absence of the PsbO protein, the high affinity binding site for calcium associated with the oxygen-evolving site was still present. These latter findings have been confirmed by the various cyanobacterial PS II crystal structures which indicate that the PsbO protein does not provide any ligands to either the calcium or manganese ions in the $\mathrm{Mn}_{4} \mathrm{CaO}_{5}$ cluster $[5,7,8,98]$.

Putative low affinity calcium-binding sites, distinct from the active site metal cluster may, however, be present on the Psbo protein, although the functional significance of these is unclear. Murray and Barber [99] identified a calcium-binding site with the calcium ligands being ${ }^{54} \mathrm{E},{ }^{114} \mathrm{E}$ and ${ }^{231} \mathrm{H}$, which was also observed by Guskov et al. [8]. It has been speculated that this low affinity site may be associated with a putative proton exit pathway leading from the $\mathrm{Mn}_{4} \mathrm{CaO}_{5}$ cluster to the surface of the complex $[7,20]$. The authors note, however, that with the exception of ${ }^{114} \mathrm{E}$, these residues are rather poorly conserved 
[99]. This calcium-binding site was not observed in the latest crystal structure [5]; while the positions of ${ }^{114} \mathrm{E}$ and ${ }^{231} \mathrm{H}$ are similar to those observed in earlier structures $[7,8,99]$, the side chain of ${ }^{54} \mathrm{E}$ is rotated away from the putative calcium-binding site and appears to associate with ${ }^{57} \mathrm{~K}$ via a charge-pair interaction. It is unclear if calcium was lost from this position in the Umena et al. structure [5] or if this putative calcium-binding site was a possible crystallization artifact in the earlier models $[7,8,99]$. A different calcium-binding site on PsbO is located in the T. vulcanus structure [5]. The putative calcium ligands are ${ }^{200} \mathrm{~N}$, the backbone carbonyls of ${ }^{138} \mathrm{~T}$ and ${ }^{201} \mathrm{~V}$ and two water molecules ( ${ }^{253}$ Wat and $\left.{ }^{1023} \mathrm{Wat}\right)$. The possible physiological significance of this site is unknown. In any event, none of the amino acid residues in either of the putative calcium-binding sites have been examined by site-directed mutagenesis in either cyanobacterial or higher plant systems.

While in Synechocystis 6803 the absence of the PsbO protein does not prevent photoautotrophy or assembly of PS II, the $\triangle p s b O$ mutant grown on complete medium [10] grew slower than wild type, evolved oxygen at about $40 \%$ wild-type rates, and was more susceptible to photoinactivation. This strain cannot grow under low calcium [100] or chloride [101] conditions. These observations indicate a role for the PsbO protein in the regulation of these ions at the oxygen-evolving site. Interestingly, in Chlamydomonas reinhardtii (hereafter C. reinhardtii) [102] and Arabidopsis [103], a profoundly different phenotype is observed. In these organisms, the absence of PsbO leads to a loss of photoautotrophic growth and the ability to assemble functional PS II reaction centers. The reason for the differences observed in the prokaryotic and eukaryotic systems is unclear at this time, although it should be noted that in the green algae and higher plants the loss of PsbO also leads to the concomitant loss of PsbP and PsbQ. In the cyanobacteria, however, the PsbU and PsbV proteins are not released upon the loss of the PsbO component. The retention of PsbU and PsbV may partially stabilize the manganese cluster in the cyanobacterial system. Another possibility is that the lumenal chloride concentration is significantly higher in cyanobacterial thylakoids than in higher plants. If this were the case, then the integrity of the $\mathrm{Mn}_{4} \mathrm{CaO}_{5}$ cluster might be maintained in the cyanobacterial system even in the absence of the PsbO protein, while a putative lower chloride concentration in the higher plant thylakoids would lead to disassembly of the $\mathrm{Mn}_{4} \mathrm{CaO}_{5}$ cluster and destabilization of PS II.

While the evidence for a chloride and/or calcium regulatory function for the PsbO component is rather strong (if circumstantial), it is also possible that the PsbO protein may participate more directly in the water-oxidizing process. In an isotope editing experiment, PsbOdepleted PS II membranes were reconstituted with uniformly ${ }^{13} \mathrm{C}$ labeled PsbO protein [104]. Fourier transform infrared spectroscopy (FTIR) was then used to monitor changes in the protonation state of the PsbO component during $S$ state turnover. During the $S_{1} \rightarrow S_{2}$ transition, spectra were obtained which were consistent with the deprotonation of the PsbO protein; the proton acceptor did not appear to reside on the protein. The authors hypothesized that PsbO may serve to stabilize the charged $\mathrm{S}_{2}$ state. Interestingly, the oxidation state of the $\mathrm{Mn}_{4} \mathrm{CaO}_{5}$ cluster appears to modify the structure of the PsbO protein [105]. Treatment of PS II membranes with $100 \mu \mathrm{M}$ hydroxylamine leads to the reduction of the $\mathrm{Mn}_{4} \mathrm{CaO}_{5}$ cluster. In the presence of the PsbO protein, the manganese and presumably calcium ions are not lost to the bulk solvent. This treatment leads to the exposure of tryptic sites on the PsbO protein. Trypsin treatment of hydroxylamine-reduced samples leads to the rapid digestion of the PsbO protein, which is normally quite resistant to trypsin attack when associated with an intact $\mathrm{Mn}_{4} \mathrm{CaO}_{5}$ cluster. This result indicates that reduction of the metal cluster leads to conformational changes in either the PsbO protein or other PS II subunits which interact with PsbO, leading to the exposure of normally trypsin inaccessible lysyl and/or arginyl residues on the PsbO protein. The locations of these residues have not been determined.
The mechanism by which the PsbO protein modulates the chloride (and possibly calcium) requirements for the photosystem is unclear. Several conserved residues on the PsbO protein have been modified by site-directed mutagenesis in spinach in an attempt to clarify this process. Modification of residues ${ }^{151} \mathrm{R}$ and ${ }^{161} \mathrm{R}$ in spinach, yielding the R151G, R151D, and R161G varients [106], lower the binding affinity of PsbO for PS II. When PsbO-depleted PS II membranes were saturated with two copies of the mutated PsbO proteins per monomer, normal sequestration of chloride was not observed and oxygen was evolved at only $20-40 \%$ of control rates. The $\mathrm{K}_{\mathrm{M}}$ for chloride was $1.5-2.5 \mathrm{mM}$ vs. $0.4 \mathrm{mM}$ for $\mathrm{NaCl}$-washed PS II membranes, which retain PsbO. No alteration for the affinity for calcium was observed. Mutations at position ${ }^{157} \mathrm{D}$ in spinach PsbO (D157N, D157K and D157E) are particularly interesting [61]. PsbO proteins with these mutations exhibit essentially normal binding characteristics when reconstituted onto PsbO-depleted PS II membranes. Nevertheless, all of these altered proteins exhibit lower affinity for chloride ( $K_{M}$ of $\left.1.5-1.6 \mathrm{mM}\right)$, no alteration in the affinity for calcium, and an inability to fully reconstitute steady-state oxygen evolution, with only about $50 \%$ of the control activity being observed. Interestingly, the D157E protein performs as poorly as the D157N and D157K variants. Additionally, fluorescence experiments indicated that the presence of these PsbO variants resulted in slow charge recombination in the presence of DCMU $\left(Q_{A}^{-} \rightarrow Q_{B}\right.$ electron transfer was not affected), indicative of a more stable $S_{2}$ state. Flash oxygen yield experiments indicated that after dark incubation a higher proportion of the PS II reaction centers were in the $S_{3}$ state [107]. Consequently, these results are similar to, but not as extreme as, results obtained from PS II membranes which lack the PsbO component or have been depleted of chloride. Popelkova and Yocum have subsequently hypothesized [53] that these residues $\left({ }^{151} \mathrm{D},{ }^{157} \mathrm{D}\right.$ and $\left.{ }^{161} \mathrm{R}\right)$ along with the additional conserved PsbO residues ${ }^{190} \mathrm{~K},{ }^{224} \mathrm{D}$ and ${ }^{226} \mathrm{D}$, interact to properly fold a domain important for chloride retention. Examination of the interactions among the analogous residues $\left({ }^{152} \mathrm{R},{ }^{158} \mathrm{D},{ }^{162} \mathrm{R},{ }^{189} \mathrm{R}\right.$, ${ }^{222} \mathrm{D}$ and ${ }^{224} \mathrm{D}$ ) in the T. vulcanus crystal structure [5] in large measure appears to confirm their hypothesis. PsbO residues ${ }^{158} \mathrm{D}$ and ${ }^{162} \mathrm{R}$ appear to form a charge-pair, while ${ }^{152} \mathrm{R}$ appears to hydrogen bond with the backbone carbonyl of ${ }^{64} \mathrm{R}$ of $\mathrm{D} 1 .{ }^{224} \mathrm{D}$ of PsbO may participate in three relevant interactions. First, its carboxylate may hydrogen bond with the backbone amide of ${ }^{310} \mathrm{E}$ of $\mathrm{D} 2$; its backbone amide appears to hydrogen bond with the carboxylate of PsbO residue ${ }^{222} \mathrm{D}$, while its backbone carbonyl forms a hydrogen bond with the backbone amide of PsbO residue ${ }^{226} \mathrm{G}$. The backbone carbonyl of this latter residue appears to hydrogen bond with both ${ }^{189} \mathrm{R}$ and ${ }^{184} \mathrm{R}$ of PsbO. It should be noted that the domain ${ }^{152} \mathrm{R}-{ }^{162} \mathrm{R}$ of PsbO closely approaches both $\mathrm{Cl}^{-} 1(10.1 \AA)$ and $\mathrm{Cl}^{-} 2(12.2 \AA)$ in the T. vulcanus crystal structure [5]. One can speculate that biochemical or genetic removal of the PsbO protein or site-directed modification of residues in the ${ }^{152} \mathrm{R}-{ }^{162} \mathrm{R}$ domain could lead to conformational changes in intervening residues, such as residues ${ }^{317} \mathrm{~K}$ of D2 and ${ }^{334} \mathrm{R}$ of D1 (including residues in the vicinity of ${ }^{64} \mathrm{R}$ which is hydrogen-bonded to ${ }^{152} \mathrm{R}$ of PsbO) at the $\mathrm{Cl}^{-} 1$ site and/or ${ }^{336} \mathrm{~A}-{ }^{338} \mathrm{~N}$ of D1 at the $\mathrm{Cl}^{-} 2$ site, which would destabilize the bound chloride and result in the multitude of chloride-related defects described above.

Within PS II, access channel(s) must exist which allow the substrate water to reach the $\mathrm{Mn}_{4} \mathrm{CaO}_{5}$ cluster and exit channels must exit which allow the products, protons and molecular oxygen, to leave the active site. A number of in silico studies have sought to identify such channels and to determine the roles played by the various PS II subunits in their formation [20,108-110]. Recently, Gabdulkhakov et al. [111] have combined in silico studies using noble gases derivatization and dimethyl sulfoxide co-crystalization to identify nine possible channels in $T$. elongatus; the channels designated $\mathrm{A} 1$ and $\mathrm{A} 2$ are putative water entrance channels, channels $\mathrm{B} 1$ and $\mathrm{B} 2$ are putative oxygen egress channels and channels $\mathrm{C}-\mathrm{G}$ are putative proton exit channels. A number of PsbO residues were predicted to be associated with water channel $\mathrm{A} 2$ and the proton exit channels $\mathrm{C}-\mathrm{E}$ and $\mathrm{G}$. The locations of these channels were calculated 
using the 2.9 A structure [8]. It will be most interesting to see similar analysis performed on the higher resolution $T$. vulcanus structure [5]. Determination of the actual functions performed by these putative channels will require additional experimentation.

\section{Psb0 as a GTPase}

Evidence has been presented that the PsbO protein binds GTP with high affinity [112] and functions as a GTPase [113]. In this role, it has been hypothesized to act to control the phosphorylation state of the D1 protein [114]. Since the phosphorylation level of the D1 component appears to be coupled with its efficient PS II turnover $[115,116]$, it was proposed that the PsbO protein may be an important regulatory component of this process [117]. In spinach, this proposed function is apparently performed by the single PsbO gene product found in this organism which also facilitates the oxygen-evolving process, as described above. The mechanisms regulating these two functions of the PsbO component are unclear. In Arabidopsis, two genes which encode the PsbO component (psbO-1, At5g66570 and psbO-2, At3g50820) are normally expressed, yielding two different PsbO proteins (PsbO-1 and PsbO-2, respectively). There are 11 amino acid differences between these two isoforms [73]. It has been hypothesized that the two functions ascribed to PsbO, support of oxygen evolution and regulation of PS II turnover, have differentiated between the two PsbO isoforms, with the primary function of the PsbO-1 protein being support of normal oxygen evolution by PS II and with the PsbO-2 protein principally acting to regulate the phosphorylation state and turnover of the D1 protein [114]. This suggestion was based largely on the observation that the PsbO-2 component exhibited substantially higher GTPase activity than the PsbO- 1 protein while functioning poorly in support of oxygen evolution [117]. The hypothesis that the PsbO protein plays an important role in PS II turnover has been evaluated critically elsewhere [118].

\section{PsbP and PsbQ}

In higher plants, the PsbP and PsbQ proteins modulate the calcium and chloride requirement for oxygen evolution. In spinach, these proteins exhibit mature molecular masses of $20.2 \mathrm{kDa}$ and $16.5 \mathrm{kDa}$, respectively [119]. Sequence alignments of these proteins from a variety of different organisms have been previously published [21]. Other members of the extended PsbP and PsbQ families have been found to function in PS II repair or as components of the chloroplastic $\mathrm{NDH}$ dehydrogenase and will not be examined in this review (for an in-depth examination of this topic, see [17-19]).

\section{Structures}

A number of groups have recently published crystal structures of PsbP from higher plants [120-122]. Fig. 3A illustrates the threedimensional structure of the PsbP protein from spinach at $1.98 \AA$ resolution (PDB ID: 2VU4, [121,122]). The structures of the spinach and tobacco (PDB ID: 1V2B, [120]) PsbP proteins and the cyanobacterial homologue CyanoP (PDB ID: 2XB3, [123]), which is shown in Fig. $3 \mathrm{~B}$, are quite similar although the cyanobacterial protein has very limited sequence homology with its higher plant counterparts [21]. The protein exhibits a two-strand anti-parallel $\beta$-sheet near its $\mathrm{N}$ terminus, and a central anti-parallel six-strand $\beta$-sheet which is flanked by two $\alpha$-helical domains. The $\mathrm{N}$-terminus of the protein is disordered and is not resolved in any of the available structures. The protein contains a number of bound zinc ions, the biological consequences of which are unclear (see below). As originally pointed out by Ifuku et al. [120], the protein shares its fold with Mog1p (PDB ID: 1EQ6 [124]), a regulatory protein which interacts with the ran GTPase in yeast [125]. This is quite interesting as the PsbP protein is

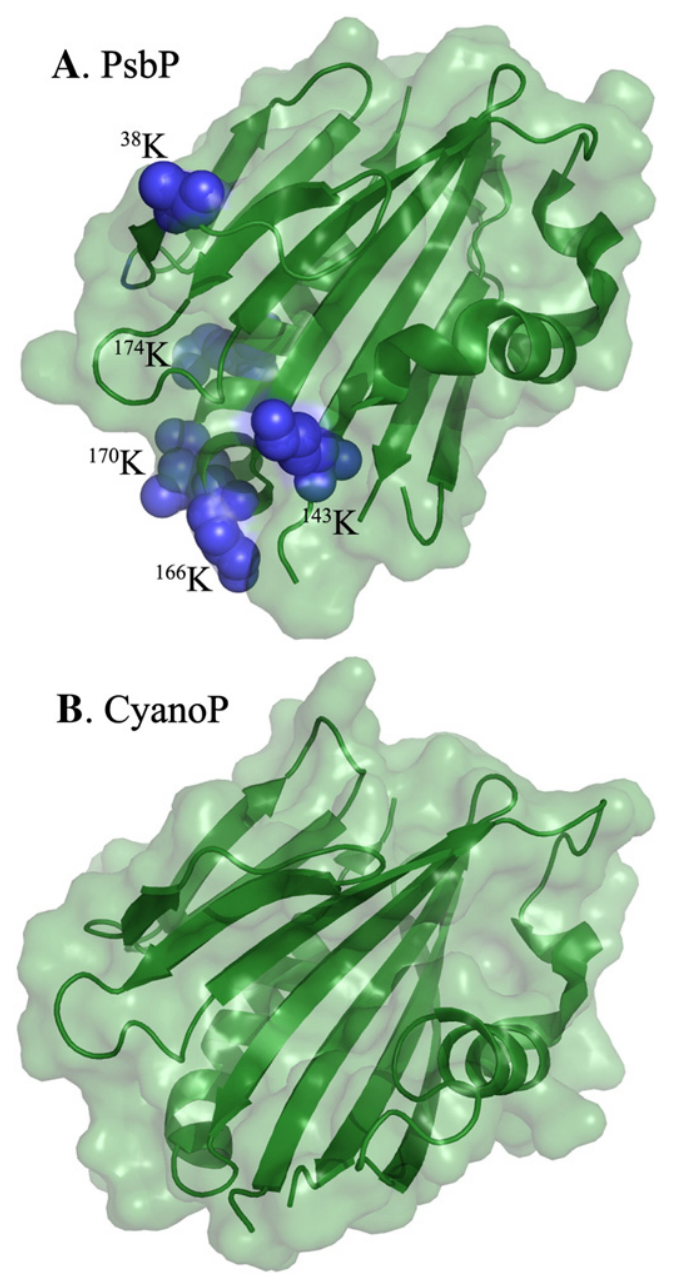

Fig. 3. Crystal Structures of PsbP and CyanoP. A., The spinach $1.98 \AA$ PsbP crystal structure (PDB ID: 2VU4 [122]) is shown. The lysyl residues identified by Tohri et al. [133] as being involved in electrostatic interactions with PS II are shown in blue. Please note that residue ${ }^{33} \mathrm{~K}$ is not visible in this rendering and is located behind and to the lower left of the molecule. B., The T. elongatus $2.8 \AA$ CyanoP crystal structure (PDB ID: $2 \mathrm{XB3}[123])$ is shown.

known to interact with the PsbO component which, as described above, has been suggested to exhibit GTPase activity [112,113].

Several groups have published crystal structures of PsbQ from higher plants [126,127]. Fig. 4A illustrates the three-dimensional structure of the PsbQ protein from spinach at $1.49 \AA$ resolution (PDB ID: 1VYK, [127]). The protein exhibits a four $\alpha$-helix bundle in its C-terminal domain which is very similar to the fold exhibited by the cyanobacterial homologue CyanoQ (PDB ID: 3LSO, [128]), as shown in Fig. 4B. A two-strand parallel $\beta$-sheet is located in the N-terminal domain along with a polyproline type II (PPII) left-handed helix formed by four sequential prolyl residues ( ${ }^{9}$ Pro- ${ }^{12}$ Pro) in the spinach protein; these features are absent in CyanoQ. The spinach protein contains a number of bound $\mathrm{Zn}^{+2}$ ions, again the function, if any, of these is unknown (see below). The loop which connects the two $\beta$ strands $\left({ }^{13} \mathrm{~L}-{ }^{34} \mathrm{~T}\right)$ is not resolved in the current structure (probably because it exhibits high mobility) but has been modeled using a molecular dynamic simulation [129].

A variety of studies have examined the association of these components to the photosystem and to each other. Early studies indicated that the PsbO protein was required for PsbP binding and that the PsbP component was required for the association of PsbQ to PS II [130,131]. Relatively few studies have addressed the mechanism of PsbP and PsbQ binding to the photosystem. Ifuku and Sato [132] reported that the binding affinity of a recombinant mutant of the PsbP protein, of which 


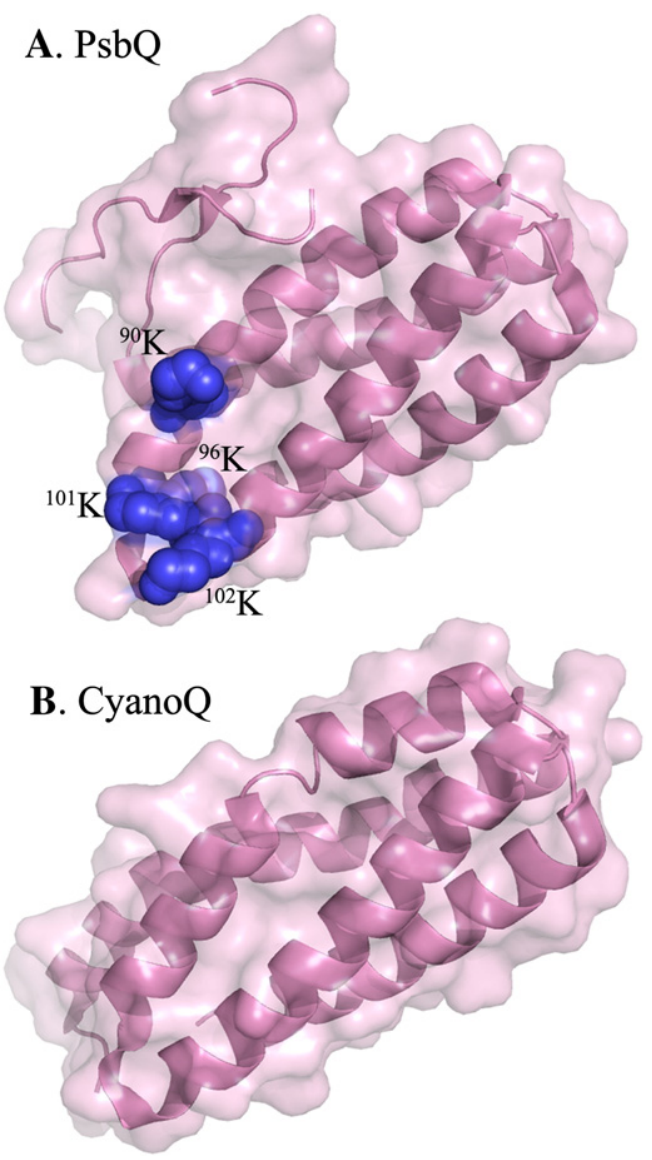

Fig. 4. Crystal Structures of PsbQ and CyanoQ. A., The spinach $1.49 \AA$ PsbQ crystal structure (PDB ID: 1VYK[127]) is shown. The lysyl residues identified by Meades et al. [136] as being involved in electrostatic interaction with PS II are shown in blue. B. The Synechocystis $68031.8 \AA$ CyanoQ crystal structure (PDB ID: 3LSO [128]) is shown.

$19 \mathrm{~N}$-terminal residues were truncated $(\Delta 19-\mathrm{PsbP})$, was weaker than that of native PsbP and did not exhibit saturation binding characteristics. Additionally, this truncated mutant exhibited functional defects (see below). The bound $\Delta 19$-PsbP protein could support the binding of the PsbQ subunit, indicating that while PsbP's binding was weaker, at least a proportion of the protein was bound normally. Unfortunately, this N-terminal domain is not resolved in the current PsbP crystal structures. Using chemical modification with N-succinimidyl propionate (NSP), which modifies primary amino groups, Tohri et al. [133] identified lysyl residues on the PsbP protein, the modification of which dramatically decreased the binding of PsbP to PS II. These residues $\left({ }^{33} \mathrm{~K}\right.$, ${ }^{38} \mathrm{~K},{ }^{143} \mathrm{~K},{ }^{166} \mathrm{~K},{ }^{170} \mathrm{~K}$, and ${ }^{174} \mathrm{~K}$ ) were clustered at the $\mathrm{N}$ - and $\mathrm{C}$-termini of the protein and were all located on one face of the PsbP protein (Fig. 3A). The residues ${ }^{11} \mathrm{~K}$ and ${ }^{13} \mathrm{~K}$ were also modified but are not resolved in the currently available structures. The authors hypothesized that a subset of these lysyl residues interact with the carboxylate residues on the PsbO protein which were identified to be required for the efficient binding of PsbP to the photosystem (see above, [134]). Interestingly, several of these residues appear to be conserved at similar positions in CyanoP $\left({ }^{42} \mathrm{R},{ }^{168} \mathrm{~K}\right.$ and $\left.{ }^{165} \mathrm{~K}\right)$.

Other investigators have attempted to identify the residues on PsbQ which are required for its association with the photosystem. Earlier work indicated that proteolytic removal of the N-terminal 12 amino acid residues of the protein abolished binding to the photosystem [135]. It was unclear, however, if this domain was sufficient to account for the interaction of PsbQ with the photosystem, and the amino acid residues responsible for the interaction were not determined. Meades et al. [136] used EDC coupled with glycine methyl ester to modify lysyl residues on the PsbQ protein. They identified four lysyl residues $\left({ }^{90} \mathrm{~K},{ }^{96} \mathrm{~K},{ }^{101} \mathrm{~K}\right.$, and $\left.{ }^{102} \mathrm{~K}\right)$ whose modification also abolished high affinity binding of PsbQ to PS II. These residues all clustered on one face of the protein and are in the vicinity of the N-terminus (Fig. 4A).

In C. reinhardtii PS II, evidence is available for the direct interaction of PsbP with PsbQ. Enami and coworkers, using the 0-length crosslinker EDC, identified a crosslinked product formed between PsbP and PsbQ [43]. ${ }^{176} \mathrm{~K}$ of the PsbP protein was identified as being crosslinked to ${ }^{28} \mathrm{D}$ of PsbQ. It should be noted that no EDC-crosslinked products have been identified between PsbP and PsbQ in higher plants. This may be due to the fact that ${ }^{28} \mathrm{D}$ is not a conserved residue in higher plant PsbQ. ${ }^{176} \mathrm{~K}$ of $C$. reinhardtii is analogous to residue ${ }^{174} \mathrm{~K}$ in spinach, which was identified in the NSP-labeling experiments described above [133]. Additionally, Nagao et al. [43] showed immunologically that PsbP and the $\alpha$ subunit of cytochrome $b_{559}$ (PsbE) were crosslinked with EDC, although the interacting residues were not identified. If this assignment is correct, then PsbP is bound to the opposite side of the PS II monomer from the PsbO protein. A direct interaction of PsbP with both PsbE and PsbO would require that PsbP occupy a position roughly similar to that occupied by PsbU and PsbV in the cyanobacterial crystal structure. This, however, would appear unlikely since the red algae contain stoichiometric amounts of PsbP, PsbQ', PsbU and PsbV [15,16] and, presumably, the PsbU and PsbV proteins in the red algae are bound to PS II in analogous locations to the PsbU and PsbV proteins in the cyanobacteria.

While most investigators believe that the standard model is correct (PsbO binding to the PS II core, PsbP binding to PsbO and PsbQ binding to PsbP), it should be noted that some results indicate that the association of PsbP and PsbQ with the photosystem may be more complicated and that the accepted model may need modification. Evidence has been presented indicating that green algal PsbP and PsbQ can bind independently of the PsbO component to the photosystem $[137,138]$.

Additionally, three groups working in higher plant systems have reported that the PsbO protein can be removed from PS II while PsbP and PsbQ remain bound to the photosystem. Differential extraction experiments using either a butanol-water phase separation system [139] or $\mathrm{HgCl}_{2}[140,141]$ indicated that PsbO could be removed from PS II preparations without the concomitant extraction of the PsbP and PsbQ proteins. In the latter studies, a moderate chloride concentration inhibited the release of the PsbO component. Unfortunately, none of these investigators demonstrated that the PsbP and PsbQ proteins were bound normally after $\mathrm{HgCl}_{2}$ treatment and could subsequently be extracted by standard procedures (i.e. $1 \mathrm{M} \mathrm{NaCl}$ treatment). It is possible that PsbO is required for the initial binding of the PsbP component but that upon binding, conformational changes occur within the intrinsic components of PS II which allow the selective removal of PsbO while PsbP and PsbQ are retained [21]. In other studies, Caffarri et al. [142] used image analysis of detergent-solubilized ( $\alpha$-D-dodecyl maltoside) PS II particles (from several Arabidopsis mutants) which were resolved by sucrose density gradient ultracentrifugation, to examine the association of the light-harvesting antennae proteins with the PS II core complex. They reported that, while PsbO is present in all PS II core-containing particles, PsbQ was present only in supercomplexes which contained Lhcb and that PsbP binding required CP26/LHCII. Unfortunately, the majority of these experiments were performed at $\mathrm{pH} 7.5$, a $\mathrm{pH}$ known to destabilize the oxygen-evolving complex. Additionally, the functional integrity of their preparations is difficult to determine since their oxygen evolution experiments also were performed at $\mathrm{pH}$ 7.5 and at sub-saturating light intensities. Nevertheless, it is possible that removal of subsets of the light-harvesting apparatus might induce conformational changes in the PS II core proteins which could alter the binding characteristics of either PsbP or PsbQ to the photosystem. 


\section{PsbP and PsbQ functions}

Many early studies have demonstrated that the PsbP and PsbQ proteins function to optimize oxygen evolution at physiological concentrations of calcium and chloride. These findings have been reviewed in detail elsewhere $[21,96,143,144]$. Briefly, removal of the PsbP and PsbQ proteins by $\mathrm{NaCl}$ treatment of PS II membranes results in a marked loss of oxygen evolution activity (to about $25 \%$ of control). Much of the lost activity can be restored by reconstitution of the extracted protein(s) to protein-depleted PS II [90,145-147], but only if the calcium and chloride cofactors are present during the reconstitution $[90,148,149]$. It should be noted that high, nonphysiological concentrations of calcium and chloride can support reasonably high rates of oxygen evolution even in the absence of the PsbP and PsbQ components [90,148,150]. Inhibition of oxygenevolving activity after extraction of the PsbP and PsbQ proteins is accelerated in the light. This appears to be due to differential binding affinities for both calcium [151,152] and chloride [153,154], which are modulated by the S-state of the oxygen-evolving complex. Depletion of the PsbP and PsbQ proteins also markedly increases the accessibility of the $\mathrm{Mn}_{4} \mathrm{CaO}_{5}$ cluster to exogenous reductants [155].

While phenomenologically the PsbP and PsbQ proteins optimize oxygen evolution under physiological calcium and chloride concentrations, the mechanism(s) of such optimization remain largely unknown. At least two possibilities exist. First, the proteins could serve to stabilize a conformation of PS II required for the normal integration of these ions into the oxygen-evolving complex. This would imply that other components of PS II exist in different conformational states which are dependent on the presence or absence of the PsbP and/or PsbQ proteins. Direct evidence for such conformational changes exists. Removal of these proteins appears to introduce alterations in the function and structure of the PS II complex. Functionally, genetic or biochemical removal of PsbP (with the concomitant removal of PsbQ) leads not only to alterations in oxygen evolution capability but also to alterations in electron transport from $Q_{A}^{-}$to $Q_{B}[156,157]$. No reducing-side defects are associated with the removal of PsbQ alone [158], at least in plants grown under normal growth light conditions. Since both the $\mathrm{Q}_{\mathrm{A}}$ and $\mathrm{Q}_{\mathrm{B}}$ sites reside on the stromal side of the membrane and PsbP is bound to the lumenal side, these results indicate that transmembrane conformational changes occur upon the removal of PsbP. Image analysis of PS II supercomplexes from higher plants indicates that removal of PsbP and PsbQ induces CP29 to move approximately $12 \AA$ towards the central core of the PS II complex [67]. This result indicates that the structural organization of the core proteins of PS II is altered by the removal of PsbP and PsbQ. The most likely candidates for such conformational changes are the D1 and/or D2 proteins, which bear the $\mathrm{Q}_{\mathrm{A}}$ and $\mathrm{Q}_{\mathrm{B}}$-binding sites. Conformational changes have also been observed directly upon PsbP binding to PS II. In an FTIR study using ${ }^{13} \mathrm{C}$-labeled PsbP, Tomita and coworkers [159] specifically probed for conformational changes in PS II arising from PsbP binding [160]. Conformational changes occurring in the vicinity of the oxygenevolving complex were identified with no identifiable changes being observed in the PsbP protein itself. When reconstitution was carried out with an N-terminally truncated PsbP ( $\Delta 15-\mathrm{PsbP})$, the conformational changes were not observed. Previously, it had been shown that the $\Delta 15$-PsbP truncated protein, while able to bind to the photosystem, could not restore normal calcium and chloride binding upon reconstitution onto PsbP-depleted PS II membranes [161]. These results indicate that the $\mathrm{N}$-terminal 15 amino acid residues of PsbP are critical in supporting normal calcium and chloride function in the photosystem. Consequently, there is a correlation between the absence of induced conformational changes near the oxygen-evolving complex, the inability to restore oxygen evolution at physiological calcium and/or chloride concentrations and the absence of the $\mathrm{N}$-terminal 15 amino acid residues of PsbP. Unfortunately, these residues are not resolved in the current crystal structures [120-122]. It should be noted that this view is complicated by the observation that PsbQ may act in a coordinated manner with PsbP with respect to calcium sequestration. This hypothesis had been suggested previously, although without direct evidence $[152,162]$. The N-terminal truncation of 19 amino acid residues from PsbP yields a protein $(\Delta 19-\mathrm{PsbP})$ which exhibits non-saturating binding characteristics and the complete loss of the ability to lower the calcium requirement for oxygen evolution [132]. If, however, native PsbQ protein is subsequently reconstituted onto $\Delta 19$-PsbP protein-containing PS II membranes, much of the oxygen evolution rate is recovered, even in the absence of added calcium. So, calcium retention can be restored in the presence of a truncated PsbP protein and, presumably, the absence of conformational alterations in the vicinity of the oxygenevolving site. If the conformational changes observed are directly related to the ability to retain calcium, then similar conformational changes should be observed after reconstitution of PS II with the truncated PsbP protein and PsbQ. The possibility of such conformational changes has not been examined experimentally.

A second possibility is that the PsbP and/or the PsbQ components actively sequester the calcium and chloride cofactors required for active and stable oxygen evolution. While no direct evidence for this possibility exists, some circumstantial evidence is available, at least with respect to the possibility that the PsbP protein can bind divalent cations like calcium. The high resolution crystal structures of both PsbP and CyanoP contain bound zinc ion(s). In tobacco and spinach PsbP [121], the zinc appears to be ligated to ${ }^{144} \mathrm{His}$ and ${ }^{165} \mathrm{Asp}$, which also corresponds to one of the four zinc-binding sites observed in CyanoP [123]. It is unclear at this time if this association is physiologically relevant or is an artifact. Three possibilities exist. First, the bound zinc could be physiologically relevant. Trace metal analysis of PS II membrane preparations from higher plants indicated that PS II membranes which contained 4 atoms of manganese per PS II also contained $1-2$ atoms of zinc per PS II $[163,164]$. The physiological consequences of the removal of this zinc from PS II have not been thoroughly investigated, although it should be noted that zinc is an inhibitor of PS II activity in both higher plants and cyanobacteria $[165,166]$. A second possibility is the PsbP protein contains a physiologically relevant divalent cation binding site which, during crystallization [123], becomes occupied by a zinc atom. The most likely ions for such an interaction would be either calcium or manganese. As noted earlier, PsbP appears to function in regulating the calcium and chloride requirements for optimal PS II activity. If, in vivo, a calcium ion was bound to this site it could conceivably donate this ion during formation of the $\mathrm{Mn}_{4} \mathrm{CaO}_{5}$ cluster or alter the calcium equilibrium in favor of bound calcium within the active site. Calcium was not present during the crystallization process in any of these studies. In this regard, it should be pointed out that it has been hypothesized that PsbP is a manganese-binding protein which delivers manganese to the oxygen-evolving site during photoactivation. This hypothesis has been critically evaluated elsewhere [118]. Finally, given the high zinc concentrations ( $5 \mathrm{mM}$ in the case of the spinach protein [121], $200 \mathrm{mM}$ in the case of CyanoP [123]) present during the crystallization of PsbP, it is possible that the zinc binding is purely an artifact of the crystallization process. Analogous arguments can be made concerning the observed bound zinc in the PsbQ and Cyano Q structures (see below).

Both PsbP and PsbQ may be phosphorylated in Arabidopsis. While no phosphorylation has been reported using standard radiolabeling procedures in any system, two recent proteomic studies have identified putative phosphorylated peptides from these two components after IMAC or $\mathrm{TiO}_{2}$ enrichment for phosphopeptides $[167,168]$. PsbP-1 and PsbP-2 were reported to contain multiple phosphorylation sites $\left({ }^{56} \mathrm{~T}\right.$, ${ }^{66} \mathrm{~T},{ }^{70} \mathrm{~S}$ and ${ }^{82} \mathrm{~S}$ of the mature protein). While phosphorylated PsbQ-1 peptides were not identified, phosphorylation of the mature PsbQ-2 protein at ${ }^{91} \mathrm{~S}$ of the mature protein was detected. Both PsbP and PsbQ 
phosphorylation was detected under both dark and light incubation conditions. The protein kinases and phosphatases associated with the observed phosphorylation have not been identified and the biological role played by the phosphorylation remains undetermined.

Finally, the PsbP and PsbQ proteins appear to function as assembly and/or stability factors for PS II in higher plants. ${ }^{9}$ These findings result from RNAi suppression experiments used to examine the effects of the loss of PsbP in tobacco [169] and Arabidopsis [156]. The results obtained in these two experimental systems are quite similar. Moderately strong suppression of PsbP (5-10\% wild-type levels) leads to slow photoautotrophic growth and a variety of functional defects associated with both the oxidizing and reducing sides of the photosystem $[156,169,170]$. The PS II reaction center proteins (CP47, CP43, D1 and D2) accumulate to relatively normal amounts, with only modest decreases being observed and with the accumulated proteins assembling into PS II dimers. LHC II-PS II supercomplexes, however, do not form and the reaction center proteins and the LHC proteins are not phosphorylated [170]. Significant defects in chloroplast and thylakoid architecture are also apparent in these plants [170,171]; however, the mechanism(s) giving rise to these alterations are unknown. Under stronger suppression of the PsbP protein $(<1 \%$ wild-type levels), a significantly different phenotype is observed. While, as expected, the various PS II functional alterations are exacerbated and the ability to grow photoautotrophically is lost, the mutant's ability to accumulate the PS II core proteins CP47 and D2 is markedly reduced [156] and the thylakoid membranes are dramatically altered [171]. It is interesting to note that the loss of the reaction center components does not parallel the loss of PsbP; even low levels of PsbP (5-10\% of wild type) support the near normal accumulation of these intrinsic components. It is only under severe depletion of PsbP that loss of the reaction center components is observed. In this respect, it is possible to hypothesize that PsbP may possess some "catalytic" function with respect to PS II assembly. The suppression of PsbP expression, while not affecting the accumulation of PsbO, leads to the complete loss of the PsbQ component, even in plants containing detectable levels of PsbP [156,169]. It is unclear at this time whether the loss of the PsbQ protein is due to decreased synthesis, inefficient transport or increased degradation. It should be noted, however, that large pools of unassembled mature PsbO, PsbQ and PsbP proteins normally exist in the thylakoid lumen without being degraded either in the presence $[172,173]$ or in the absence $[174,175]$ of assembled and functional PS II reaction centers. Consequently, the loss of the PsbQ component must be the direct result of the loss of expression of the PsbP protein and not a consequence of the reduced levels of PS II reaction centers observed when the PsbP component is strongly suppressed.

The PsbQ protein has also been examined using RNAi suppression techniques in both tobacco [169] and Arabidopsis [158]. Under normal growth light conditions, the complete suppression of PsbQ expression did not affect photoautotrophic growth, PS II assembly, or most PS II functional parameters (oxygen evolution rate, $\mathrm{F}_{\mathrm{V}} / \mathrm{F}_{\mathrm{M}}$, etc.) in either system. In Arabidopsis some modest defects were observed with respect to $\mathrm{S}$-state distribution and the stability of the oxygen-evolving complex under dark incubation conditions [158]. A major alteration in phenotype was observed, however, when Arabidopsis mutants lacking the PsbQ proteins were placed under low light conditions $(5 \mu \mathrm{mol}$ photons $\left.\cdot \mathrm{m}^{-2} \cdot \mathrm{s}^{-1}\right)$. After three weeks these plants yellowed and died while wild-type plants were unaffected. The PsbQ-deficient plants lost the ability to assemble functional PS II reaction centers, with major losses of the CP47, CP43 and D2 components being observed. Interestingly the D1 protein was not seriously affected. The functional characteristics of the PS II reaction centers which did assemble were also seriously compromised [158]. A more detailed

\footnotetext{
${ }^{9}$ In cyanobacteria the loss of CyanoP or CyanoQ does not dramatically affect PS II assembly or stability.
}

examination of the assembly characteristics of PsbP- and PsbQdeficient higher plants and a comparison between the higher plant, C. reinhardtii and cyanobacterial model systems is presented elsewhere [118].

\section{PsbR}

The PsbR protein is the most enigmatic of the extrinsic proteins associated with higher plant and green algal PS II; genes encoding this component are not found in the genomes of cyanobacteria or red algae. PsbR was first identified in the PS II complex of spinach [176]. Higher plants utilize a poorly understood targeting mechanism for this component. PrePsbR, which is translated on cytosolic ribosomes, has a very short transit sequence (only 41 amino acid residues in both Arabidopsis and spinach) relative to the other nuclear-encoded extrinsic proteins of the PS II complex. For instance, the PsbO-1 protein in Arabidopsis contains an 85 amino acid residue bipartite transit sequence. It has been suggested [177] that the shortened $\mathrm{N}$-terminal sequence acts to target PsbR to the chloroplast stroma while a C-terminal hydrophobic domain of the protein functions as a non-cleavable signal for lumenal transport. This domain would subsequently act as a hydrophobic anchor of the PsbR protein to the lumenal side of the thylakoid membrane, although this hypothesis has not been tested experimentally. The hydrophobic nature of the C-terminus of the PsbR protein apparently makes this component relatively insoluble and the protein tends to precipitate during isolation [178]. Interestingly, the protein is removed from PS II membranes by alkaline-Tris washing of PS II membranes $[176,178]$. This almost certainly indicates that the hydrophobic C-terminal domain is not organized as a transmembrane $\alpha$-helix since proteins with a single transmembrane helix, such as cytochrome $f$, are not removed by this treatment.

It is unclear which subunits are the nearest neighbors of PsbR within the PS II complex. Since PsbR is not present in cyanobacterial PS II, no crystallographic information is available. The only experimental structural information is from the analysis of protein crosslinking experiments [179] using 3,3'-dithiobis[sulfosuccinimidylpropionate], a cleavable crosslinker which spans 12 Å. In this study, CP47 formed a crosslinked species with an unidentified $10 \mathrm{kDa}$ polypeptide, yielding a $54-56 \mathrm{kDa}$ product. The authors tentatively identified the $10 \mathrm{kDa}$ component as the PsbR protein due to its apparent molecular weight and the disappearance of the crosslinked species when the PS II membranes were Tris-washed prior to the initiation of the crosslinking experiment. PsbR appears to be required for PsbP assembly into the PS II complex. Additionally, PsbJ, a low molecular mass plastid-encoded hydrophobic subunit, appears to be required for the stable assembly of PsbR [180] and, consequently, PsbP [181,182]. One possible model would be that PsbJ serves to anchor the PsbR protein to the PS II complex (possibly by interacting with the hydrophobic C-terminus of PsbR) and that PsbR participates along with PsbO in the formation of a binding domain for PsbP. If this model is correct, PsbR would be positioned at approximately the location of PsbV in cyanobacteria ${ }^{10}$ [5].

In the absence of crystallographic information, I-TASSER was used to predict the three-dimensional structure of the PsbR protein $[183,184]$. I-TASSER was the best performing structural prediction software platform on the CASP-7, CASP-8 and CASP-9 threedimensional structural prediction trials. The top two models provided by I-TASSER are shown in Fig. 5. While such models must be considered tentative, in all of the predicted structures examined (top five models) the C-terminal domain is predicted to be organized as a hydrophobic helix-coil-helix motif. The longest helical domain averaged about fourteen amino acid residues in length. It is unlikely that such a structure could span the thylakoid membrane and it is

\footnotetext{
${ }^{10}$ In this regard it should be noted that the closest approach of PsbV to CP47 in cyanobacteria is less than $12 \AA$ [5]. If PsbR occupies nearly the same position in higher plants, this could be consistent with the crosslinking evidence presented in [179].
} 
possible that this anchors PsbR to the membrane (or to hydrophobic protein subunits such as PsbJ), which would be consistent with its release by alkaline-Tris treatment $[176,178]$.

The function of the PsbR protein in vivo has been investigated using mutant plants in both potato [185] and Arabidopsis [180,186,187]. In these reports, the absence of the PsbR protein was reported to lead to impaired oxygen evolution and $\mathrm{Q}_{\mathrm{A}}^{-}$reoxidation. These defects may be directly related to the inability of the PsbP proteins to bind to PS II in the absence of the PsbR protein. In initial studies, the absence of PsbR did not appear to affect the accumulation of other PS II components. Specifically, in whole leaf extracts, the amounts of immunologically detectable PsbP and PsbQ proteins were not affected [185]. Later studies, however, examining thylakoids $[180,186]$ or PS II membranes [187] isolated from mutants lacking the PsbR component, indicated that the PsbP and PsbQ proteins were strongly depleted. It was hypothesized that the absence of the PsbQ component was a secondary effect subsequent to the failure of the PsbP protein to bind in the absence of PsbR [180]. Similar electron transport defects have been documented in PS II which has been depleted of the PsbP component either genetically [156] or biochemically [157].

In C. reinhardtii, the PsbR protein is differentially phosphorylated [188]. Under State 1 conditions (aerobic dark incubation) no phosphorylation is observed while under State 2 conditions (anaerobic dark incubation) or incubation under moderate or high light, the protein is phosphorylated. The phosphorylation site is ${ }^{43} \mathrm{~S}$ of the initial translation product (residue ${ }^{15} \mathrm{~S}$ of the mature protein). In higher plants, this phosphorylation site is not conserved and using standard ${ }^{32} \mathrm{P}$ radiolabeling techniques, PsbR is not observed to be phosphorylated. Recently, however, two proteomic studies examining the phosphoproteome of Arabidopsis have detected putative PsbR phosphorylation using MS/MS techniques. The protein was reported to be phosphorylated under both light and dark conditions at ${ }^{58} \mathrm{~S}$ (residue ${ }^{30} \mathrm{~S}$ of the mature protein) $[167,189]$. The identity of the protein kinase and phosphatase associated with this phosphorylation site are unknown [190]. The function of the differential phosphorylation in C. reinhardtii and the possible phosphorylation in higher plants is unknown.

\section{PsbU}

\subsection{Structure}

The X-ray-derived crystal structure of bound PsbU has been determined in T. vulcanus [5,191] and T. elongatus [7,8,98]. The structure of PsbU from T. vulcanus at a resolution of $1.9 \AA$ is shown in Fig. 1 in blue and possesses six alpha helices connected by five loops with little or no $\beta$-sheet structure being recognizable. No functional domains or other structural homology exist to other proteins in the current databases. The interacting partners in the photosystem include CP47, PsbO and PsbV.

PsbU was first identified in the cyanobacterium Phormidium laminosum as an $\sim 9 \mathrm{kDa}$ polypeptide [192] and was subsequently shown to be a stoichiometric component of cyanobacterial PS II [193]. The first report of this protein in a eukaryotic alga was in isolated PS II preparations from the red alga Cyanidium caldarium (henceforth, C. caldarium) [194]. Unlike PsbV, PsbU is nuclear-encoded in the red algae $[195,196]$. The PsbU subunit has been identified in most cyanobacteria, with the exception of Prochlorococcus marinus strains MED4 and SS120, although it is present in P. marinus MIT9313. This subunit has also been detected in chlorophyll $a / c$-containing algae $[197,198]$. In contrast, PsbU is absent from all chlorophyll $a / b$-containing eukaryotes.

The binding of PsbU to PS II was initially studied in isolated PS II complexes from T. vulcanus $[193,199]$. The PsbU protein was not able to bind to PS II in the absence of PsbO and PsbV. PsbU was observed to bind at low levels if only one of these polypeptides was present [199].
PsbU bound at approximately 40\% its native level when only PsbV was present and $25 \%$ of its native level when only PsbO was present. Furthermore, oxygen evolution by isolated PS II complexes in which the three extrinsic proteins had been removed by a $\mathrm{CaCl}_{2}$ treatment was greatly reduced and required reconstitution with PsbO, PsbV and PsbU to fully restore activity. Partial restoration of activity in the absence of PsbU, however, was observed [199].

Gene deletion and gene interruption studies with Synechocystis 6803 found the $\Delta p s b V$ mutant and the $\Delta p s b V: \Delta p s b U$ double mutant had indistinguishable phenotypes, both consistent with PsbU requiring the presence of PsbV to functionally associate with PS II [200,201]. Interestingly, while the $\Delta p s b V: \Delta p s b U$ double mutant was able to grow photoautotrophically, the $\Delta p s b 0: \Delta p s b U$ double mutant was not - at least when the cells were grown at $\mathrm{pH}$ 7.5. Photoautotrophy could be restored to the $\Delta p s b 0: \Delta p s b U$ double mutant when cells were grown at $\mathrm{pH} 10$ [201]. Although the mechanistic basis for this pH-dependent growth has proved difficult to pinpoint [202] it does appear consistent with the in vitro binding studies performed with T. vulcanus, since in those reconstitution studies, PsbU bound with twice the affinity to complexes having only PsbV compared with complexes having only PsbO [199].
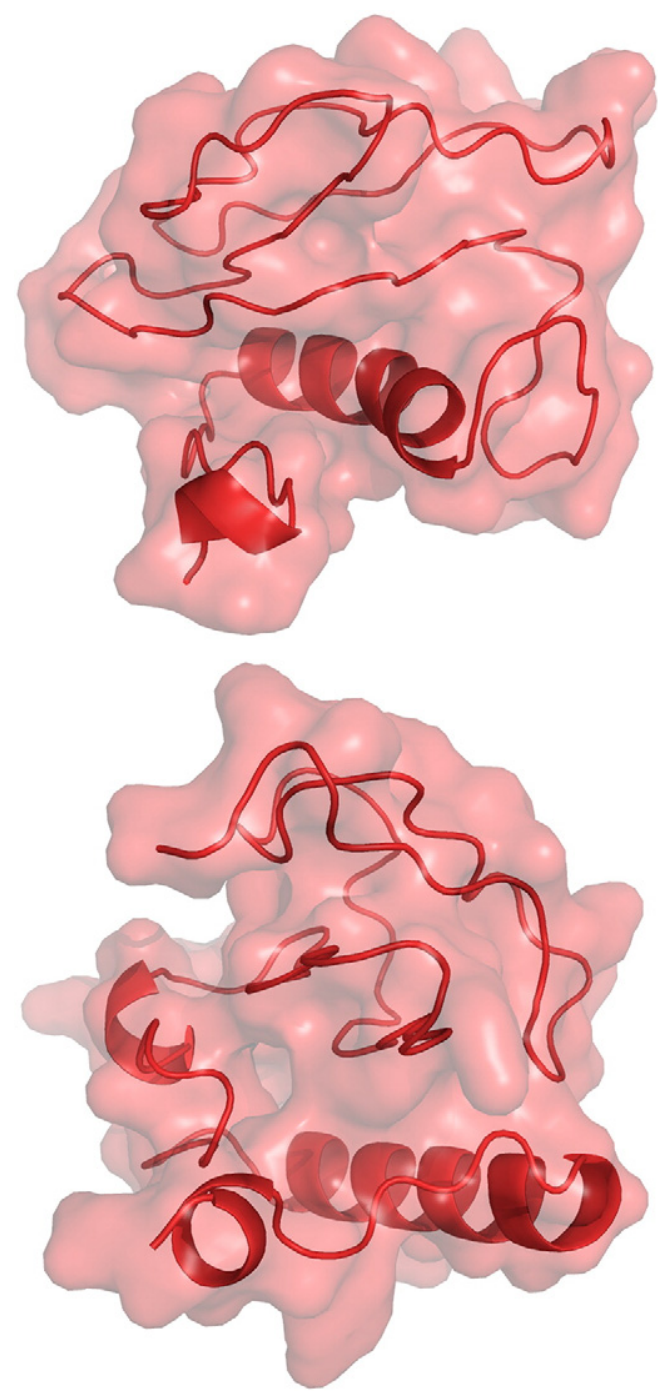

Fig. 5. Putative structures for PsbR. The structure of PsbR was modeled using the program I-TASSER $[183,184]$. The top two candidate structures are illustrated. Both exhibited a helix-coil-helix motif at their C-termini. The longest helical segment averaged 12-15 amino acid residues in length in five candidate structures examined. This domain is probably too short to span the thylakoid membrane. 


\subsection{Function}

Synechocystis 6803 cells lacking PsbU are photoautotrophic when grown under standard laboratory conditions in BG-11 growth medium and assemble PS II centers at a similar level to wild type $[200,203,204]$. While photoautotrophic growth rates of the mutant and wild type are similar, the $\Delta p s b U$ cells have a more stable $S_{2}$ state $[200,204,205]$, although the consequences of this for the viability of the cells are not immediately obvious. In the absence of PsbU, oxygen evolution rates are reduced by typically $20 \%$ or more relative to wild type and photoinactivation is observed during steady-state oxygen evolution measurements. This is consistent with the observation that $\Delta p s b U$ mutants are susceptible to photodamage when exposed to high light, exhibiting a more rapid degradation of the D1 protein [200,203,204,206-208].

In addition to the above PS II-specific perturbations, the $\Delta p s b U$ mutants are more susceptible to heat stress. Heat-inactivation of PS II is known to involve the loss of manganese from the oxygen-evolving complex [209]. Addition of PsbU to isolated thylakoid membranes from Synechococcus sp. PCC 7002 (henceforth Synechococcus 7002) that had been depleted of this subunit increased the heat stability of oxygen evolution while inactivation of $p s b U$ in Synechococcus 7002 prevented the ability of these mutants to acclimate to high temperature. In the absence of PsbU, the cells were unable to acquire cellular thermotolerance [210-212]. A similar result was obtained with a $\Delta p s b U$ strain of Synechocystis 6803, although in this cyanobacterium the removal of PsbU only partially prevented acclimation to high temperature, whereas cells lacking PsbO or PsbV failed to develop cellular thermotolerance when grown at moderately high temperatures [206]. These results indicate that the stability of PS II, conferred by the lumenal extrinsic proteins, is important for cells to acquire thermotolerance by optimizing specific protein-protein or protein-lipid interactions; however, the mechanism is as yet unidentified.

Interestingly, removal of PsbU in Synechocystis 6803 also resulted in uncoupling of energy transfer from the phycobilisome to the photosystem. $\Delta p s b U$ cells exhibited a decreased chlorophyll $a$ variable fluorescence yield when excited with light absorbed by allophycocyanin but not when excited by light absorbed primarily by chlorophyll a [203,207]. Furthermore, picosecond fluorescence decay kinetics revealed changes in both allophycocyanin- and PS IIassociated decay components, consistent with a decrease in the coupling of phycobilisomes to PS II and an increase in the number of closed PS II reaction centers in the dark-adapted $\Delta p s b U$ mutant [207]. Thus removal of PsbU introduces PS II-specific structural changes on both sides of the thylakoid membrane.

Inactivation of PsbU has also been studied in Synechococcus sp. PCC 7942 (henceforth, S. elongatus) [208]. These experiments demonstrated an increased rate of D1 degradation under both low and high light conditions. In addition, the recombination reaction between the reduced primary quinone acceptor $\mathrm{Q}_{\mathrm{A}}^{-}$and the $\mathrm{S}_{2}$ state in the presence of DCMU was inhibited by up to 5 -fold in the mutant, which is perhaps consistent with the long-lived $\mathrm{S}_{2}$ state observed in the $\Delta p s b U$ mutant of Synechocystis 6803 [200,204]. However, the thermoluminescence signal in the $\Delta p s b U$ mutant of $S$. elongatus was greatly diminished compared to that observed in Synechocystis 6803 [199]. This result is difficult to interpret and could possibly be a result of uncoupling of the phycobilisome from the reaction center, as reported to be the case in Synechocystis $6803 \Delta p s b U$ cells [207] or irreversible inactivation of PS II in the S. elongatus mutant at the sub-zero temperatures utilized in the thermoluminescence assay. The suggestion that the observed thermoluminescence signal might arise from the loss of additional protein subunits in the mutant seems less likely since oxygen evolution rates were reported to be similar in both the $\Delta p s b U$ mutant and wild type [208]. However, the most striking result reported for the $\Delta p s b U$ S. elongatus strain was the viability of the mutant cells when challenged by $\mathrm{H}_{2} \mathrm{O}_{2}$ or methyl viologen. Compared to wild type, the mutant exhibited greater resistance and appeared to up-regulate antioxidant enzymes. This study suggests that perturbation of PS II activity by removing PsbU may increase the production of reactive oxygen species. Consequently these mutants may be more resistant when challenged by an external oxidative stress because their endogenous protective mechanisms are already activated [208].

The requirements for the chloride and calcium cofactors in PS II are modulated by the extrinsic proteins of the photosystem [213]; the effects of omitting these ions have been investigated in $\Delta p s b U$ strains of Synechocystis 6803. Photoautotrophic growth in calcium-limiting medium was similar for wild type and the $\Delta p s b U$ mutant; however, under chloride-limiting conditions, growth was retarded in the $\Delta p s b U$ strain. Additionally, photoautotrophic growth was abolished for the $\Delta p s b U$ strain when both $\mathrm{Ca}^{2+}$ and $\mathrm{Cl}^{-}$were omitted from the growth medium [39,200,204]. In the 1.9 A resolution structure of PS II from $T$. vulcanus two chloride ions have been identified in the vicinity of the $\mathrm{Mn}_{4} \mathrm{CaO}_{5}$ cluster of the oxygen-evolving complex and may contribute to the maintenance of the coordination environment of the cluster [5]; hence it is possible that structural rearrangements resulting from removing PsbU might affect the integrity of these chloride-binding sites.

The two chloride binding sites identified in PS II from T. vulcanus are positioned at the start of hydrogen bond networks arising from the $\mathrm{Mn}_{4} \mathrm{CaO}_{5}$ cluster and extending towards the bulk solution. The network through the $\mathrm{Cl}^{-} 1$ site is located in the interface of the D1, D2 and PsbO subunits, while the $\mathrm{Cl}^{-} 2$ site is located at the interface of D1, CP43 and PsbU subunits. These channels may function as proton exit channels or water inlet channels and hence removing PsbU may disrupt these processes, which in turn may contribute to the phenotype of the $\Delta p s b U$ mutants. The presence of a putative oxygen exit channel in the vicinity of PsbU in T. vulcanus is shown in Fig. 6 and would correspond to the putative B2 oxygen exit channel suggested by Gabdulkhakov et al. [111]. The channel is formed by contributions from the D1, D2, CP43, CP47, PsbU and PsbV subunits with the mouth of the channel composed of residues from PsbU, PsbV and D2.

A similar role for PsbU has been suggested following analysis of the X-ray-derived PS II structure from T. elongatus at 2.9 Å resolution [111]. Theoretical calculations suggested the presence of several channels potentially associated with water access, proton exit and oxygen exit. The two channels suggested to play a role in oxygen exit received experimental support by co-crystallization with the noble gas krypton as well as dimethyl sulfoxide, which were both shown to localize to the putative channels. One of the exit channels was blocked by the Cterminal lysyl residue of PsbU $\left({ }^{134} \mathrm{~K}\right.$ in PsbU from T. elongatus) and the authors proposed that the $\mathrm{C}$-terminus of PsbU might operate in a gating mechanism for this channel. While the lysyl residue at this position is not absolutely conserved, an arginyl residue is frequently present that would preserve the required hydrogen bonding network; however, some PsbU sequences do not retain a positive charge at their C-terminus, and therefore the significance of this proposal requires further experimental investigation. Indeed, mutation of this terminal lysyl residue in vitro employing PS II isolated from the red alga $C$. caldarium did not produce any detectable phenotype [214].

Although the C-terminal lysyl residue of PsbU is not always present, the adjacent tyrosyl residue $\left({ }^{103} \mathrm{Y}\right)$ is conserved in both prokaryotic and eukaryotic organisms. Mutation of this residue dramatically altered the calcium and chloride requirements for oxygen evolution and resembled a PsbU-minus phenotype even though the binding of PsbU was unaffected. Moreover, only phenylalanine can partially substitute for tyrosine at this position suggesting that an aromatic group is required [214,215]. Okumura and co-workers have proposed that ${ }^{103} \mathrm{Y}$ interacts with ${ }^{340} \mathrm{P}$ located near the C-terminus of the D1 protein and it is the disruption of this interaction that results in the elevated requirement for calcium and chloride in the absence of PsbU or upon mutation of the tyrosyl residue $[214,215]$. It is therefore plausible that the pleiotrophic effects 


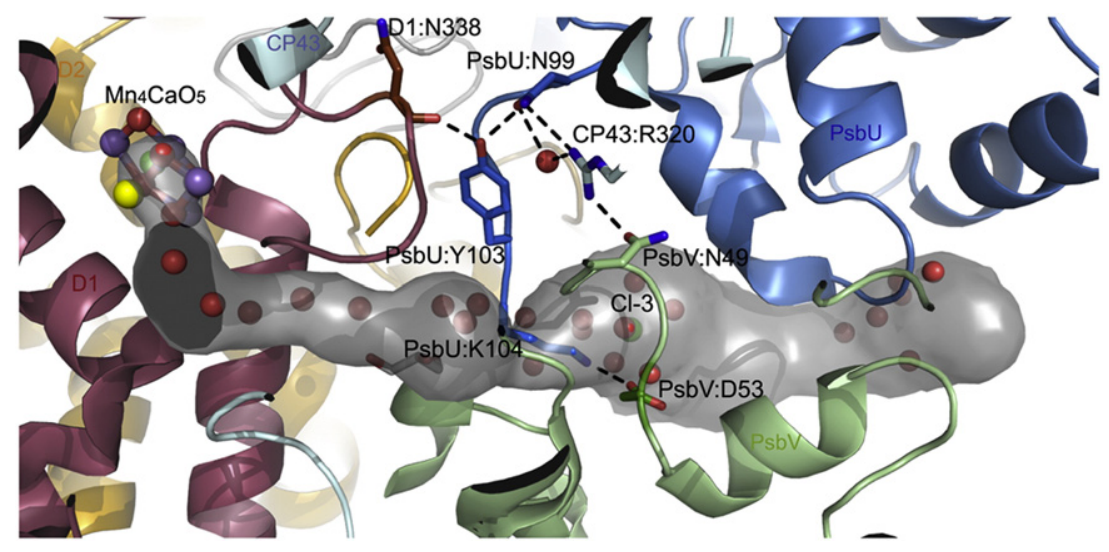

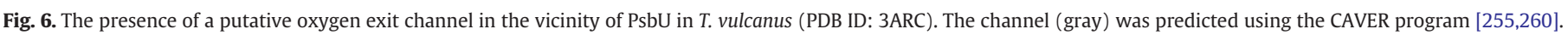

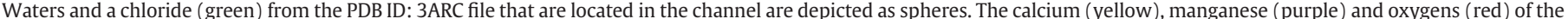

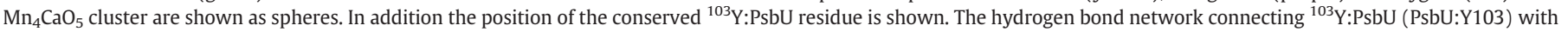
${ }^{320} \mathrm{R}: \mathrm{PsbC}$ (CP43:R320) is indicated (dotted black lines). See text for additional details.

of removing PsbU from PS II manifest themselves through structural changes that include alteration of the chloride-binding site(s) which, in turn, can perturb the environment of the $\mathrm{Mn}_{4} \mathrm{CaO}_{5}$ cluster, as well as altering additional protein-protein or protein-lipid interactions within the photosystem. Additionally, ${ }^{103} \mathrm{Y}$ may indirectly interact with ${ }^{320} \mathrm{R}$ of $\mathrm{CP} 43 .{ }^{320} \mathrm{R}: \mathrm{CP} 43$ hydrogen bonds with ${ }^{99} \mathrm{~N}$ :PsbU (moreover a second hydrogen bond between these two residues is also apparent via an intervening water: see Fig. 6) and this residue forms a hydrogen bond with ${ }^{103} \mathrm{Y}$ :PsbU. ${ }^{320} \mathrm{R}: \mathrm{CP} 43$ also hydrogen bonds with ${ }^{49} \mathrm{~N}$ :PsbV. Within this context, mutation of the analogous Synechocystis $6803 \mathrm{CP} 43$ lysyl residue $\left({ }^{305} \mathrm{R}\right)$ yields a mutant (R305S) which, while growing at a normal rate in complete medium, exhibited a loss of oxygen evolution capability (70\% of control), assembled $30 \%$ fewer PS II reaction centers and was more susceptible to photoinactivation [216]. Under chloride-limiting conditions this mutant grew very slowly, evolved oxygen at only $20 \%$ of the control rate, and exhibited a long-lived $S_{2}$ state [217]. PS II particles isolated from this mutant lost the ability to bind PsbV [218] and PsbU (T. M. Bricker and C. Putnam-Evans, unpublished). These results indicate that this CP43 arginyl residue may be involved in the formation of a high-affinity binding site for both PsbU and PsbV.

Isolated PS II from C. caldarium has been used extensively to investigate the role of PsbU and this has established that the binding of PsbU moderates the requirements for calcium and chloride by minimizing the concentrations of these cofactors that are required for maximum activity [14,214]. Hence the requirements for calcium and chloride upon removal of PsbU in red algae and cyanobacteria are similar; however, while the PsbQ orthologue (PsbQ'), PsbU and PsbV from $C$. caldarium are able to bind to T. vulcanus PS II, leading to restoration of oxygen evolution, the cyanobacterial PsbU and PsbV from T. vulcanus only partially rebind to C. caldarium PS II and are unable to restore PS II activity [219]. Therefore, while the extrinsic protein environments of red algae and cyanobacteria are much alike they are not identical. Nevertheless, red algal PsbU can functionally bind to PS II from T. vulcanus in the presence of either the cyanobacterial or red algal PsbV proteins, although cyanobacterial PsbU cannot reconstitute red algal PS II even in the presence of cyanobacterial PsbV [220].

\section{PsbV}

PsbV, also referred to as cytochrome $c_{550}$, is a PS II lumenal extrinsic subunit found in many cyanobacteria and red algae. It is a c-type monoheme cytochrome with a molecular weight of approximately $15 \mathrm{kDa}$ and an acidic isoelectric point. The existence of a lowpotential cytochrome in cyanobacterial species had long been known [221], but PsbV was first identified as a stoichiometric PS II component in isolated core complexes from T. vulcanus [222]. Since then, PsbV has been the focus of extensive research aimed at understanding its contribution to PS II oxygen-evolving activity given the unusually low midpoint potential $\left(E_{m}\right)$ of its heme group.

\subsection{Structure}

A number of structural studies have been performed on soluble PsbV from different cyanobacteria including Synechocystis 6803, Arthrospira maxima, and T. elongatus [223-225]. The PsbV heme is covalently bound to two cysteinyl residues $\left({ }^{37} \mathrm{C}\right.$ and $\left.{ }^{40} \mathrm{C}\right)$ and noncovalently coordinated by two histidyl axial ligands $\left({ }^{41} \mathrm{H}\right.$ and $\left.{ }^{92} \mathrm{H}\right)$ [223-225]. This bis-histidyl ligation scheme is somewhat unusual for $c$-type cytochromes. However, the rest of the protein structure is quite typical of other cytochromes, with a hydrophobic inner core of the protein forming a pocket for the prosthetic group and generally protecting it from the solvent, with the exception of an exposed edge including rings $\mathrm{C}$ and $\mathrm{D}$ along with the $\mathrm{D}$ propionate group.

PsbV has also been structurally resolved as a component of PS II crystal structures (see Fig. 1, green subunit) from thermophilic cyanobacteria $[5,7,8,98,191]$, and the soluble and PS II-bound PsbV protein structures are quite similar. Within the context of cyanobacterial PS II, the PsbV protein is located near lumenal loop regions of the CP43 (residues 386-397 and 412-417) and D1 (residues 305-315) proteins [5]. The ${ }^{320} \mathrm{R}$ residue of $\mathrm{CP} 43$ also comes to within $3 \AA$ of residues ${ }^{49} \mathrm{~N}$ and ${ }^{51} \mathrm{~S}$ on PsbV. This is significant because site-directed modification of the analogous residue in Synechocystis 6803 (CP43 ${ }^{305} \mathrm{R}$ ) exhibited markedly reduced PsbV binding to PS II [218]. In the current PS II structure calcium and chloride ions can each be observed at the periphery of PsbV, but the closest residues to these ions on PsbV are more than $4 \AA$ away. The $C$ and $D$ rings of the heme group are shielded by the large lumenal loop region of the CP43 protein, but there is a small opening in the protein surface that exposes the propionate oxygen of the $\mathrm{D}$ ring. The closest edge of the heme group is approximately $23 \AA$ away from the nearest manganese atom of the $\mathrm{Mn}_{4} \mathrm{CaO}_{5}$ cluster, which is the nearest redox cofactor to the PsbV heme in PS II [5]. Residues of the PsbV protein $\left({ }^{137} \mathrm{Y}\right.$ and $\left.{ }^{129} \mathrm{~K}\right)$ have also been implicated in a hydrogen bond network linking $\mathrm{Y}_{Z}$ and the bulk solvent on the lumenal side of the complex. This channel, formed by residues of the D1, CP43 and PsbV proteins, may serve as a proton exit channel for proton-coupled electron transfer via $\mathrm{Y}_{Z}[5]$.

The binding properties of PsbV differ between cyanobacteria and red algae. Reconstitution experiments in T. vulcanus revealed that the PsbV protein could bind to PS II complexes independently of the PsbO protein, but this association did not support oxygen-evolving activity. The presence of both the PsbO and PsbU proteins was necessary for 
full rebinding and maximal oxygen evolution rates [199]. PsbV was also identified in PS II preparations of the red alga $C$. caldarium $[194,226]$. In contrast, PsbV could not bind independently of the other extrinsic proteins in red algae [15]. Cross-reconstitution studies found the red algal PsbV protein to be functionally exchangeable between the cyanobacterial and red algal PS II systems. However, this was not true for the cyanobacterial PsbV protein [220]. Thus, while the PsbV proteins from cyanobacteria and red algae are quite similar (40-60\% identical), the binding sites of the PS II membrane components are apparently quite different between the two systems [220].

\subsection{Function}

Subsequent mutant studies in cyanobacteria further solidified the link between the PsbV protein and PS II oxygen evolution. A Synechocystis 6803 mutant lacking PsbV $(\Delta p s b V)$ has reduced accumulation of PS II in the thylakoids (60\% that of wild type) and lower oxygen evolution rates (40\% relative to wild type) [227]. Consequently, the $\Delta p s b V$ mutant exhibits severe growth phenotypes. The doubling time for photoautotrophic growth of $\Delta p s b V$ is more than twice that of wild type, and $\Delta p s b V$ cells cannot grow when calcium and chloride are eliminated from the growth medium [205,228]. These results suggest that PsbV aids in maintaining the proper ion environment within the oxygen-evolving complex. In this respect, PsbV functions analogously to PsbP in higher plants. Flash oxygen yield experiments have also shown an increase in the miss and double hit factors and slowed $\mathrm{S}_{3} \rightarrow\left[\mathrm{S}_{4}\right] \rightarrow \mathrm{S}_{0}$ transition in mutants lacking the PsbV protein [205]. The photoautotrophic growth and steady state oxygen evolution rates of a T. elongatus $\Delta p s b V$ mutant were less severe relative to that of the Synechocystis $6803 \Delta p s b V$ mutant [229]. Nevertheless, the T. elongatus $\triangle p s b V$ mutant was unable to grow in medium depleted of either calcium or chloride [229,230].

Additionally, the overall stability of PS II is reduced in the absence of PsbV. Dark treatment as well as heat reduces photosynthetic capacity more dramatically in the Synechocystis $6803 \Delta p s b V$ mutant [205]. This correlates with previous biochemical work that identified PsbV as the component responsible for heat stability of PS II oxygen evolution in Synechococcus 7002 [210]. However, the T. elongatus $\triangle p s b V$ mutant did not display the PS II instabilities upon dark incubation or heat treatment as had been reported for the Synechocystis 6803 mutant [230]. The authors postulate that some of the functional defects observed in the Synechocystis $6803 \Delta p s b V$ mutant may also be attributed to weaker association of the PsbO protein to PS II in these cells. The $\Delta p s b O \Delta p s b$ and $\Delta c y a n o Q \Delta p s b V$ double mutants in Synechocystis 6803 do not grow photoautotrophically in standard medium [39,227]. Therefore, the combined loss of PsbV and either PsbO or PsbQ has disastrous consequences for PS II stability and function.

Interestingly, several species of cyanobacteria (T. elongatus, T. vulcanus, Acaryochloris marina MBIC11017, Arthrospira plantensis NIES-39, and Arthrospira maxima) contain a second copy of the $p s b V$ gene, denoted $p s b V 2$. In these cyanobacterial genomes, the $p s b V 2$ gene is adjacent to the $p s b V$ (or $p s b V 1$ ) gene, and, in some cases, these genes cluster with petJ, which encodes cytochrome $c_{6}$ [229]. While the PsbV2 protein has $\sim 45 \%$ sequence identity with that of PsbV1, it lacks the second histidine residue that is normally the sixth axial ligand to the heme group, and it is likely that a tyrosine residue $\left({ }^{86} \mathrm{Y}, \mathrm{T}\right.$. elongatus numbering) provides this ligand in the PsbV2 protein [225]. In T. elongatus cells, psbV2 expression can be detected, albeit at very low levels ( $1 \%$ that of $p s b V 1)$, indicating that PsbV2 does not significantly contribute to PS II function under normal conditions [225]. Heterologous expression of T. elongatus psbV2 in Synechocystis $6803 \Delta p s b V$ mutant cells restored wild-type photoautotrophic growth rates in complete medium. However, psbV2 expression could not restore photoautotrophic growth in medium lacking calcium or chloride [229]. Notably, the T. elongatus psbV1 gene was able to fully complement the Synechocystis $\Delta p s b V$ mutant phenotypes.
Additionally, Synechocystis $6803 \Delta p s b V$ mutant cells heterologously expressing $p s b V 1$ contained detectable amounts of heme corresponding to cytochrome $c_{550}$; however, no heme was detected in cells expressing only psbV2[231]. While there is no experimental evidence that the heme group of PsbV participates in electron transfer reactions within PS II (see below), it is intriguing that the absence of the heme group in the PsbV2 protein correlates with an inability to fully complement the $\Delta p s b V$ mutant phenotype.

\subsection{Midpoint potential}

The $E_{m}$ of PsbV and its functional significance has been the subject of intense research. Unlike other c-type monoheme cytochromes, which have an $E_{m}$ of $+0 \mathrm{mV}$ or greater [232], the $E_{m}$ of soluble PsbV has been reported to be approximately $-250 \mathrm{mV}$ in numerous cyanobacterial species [221,230,233,234]. A variety of roles have been proposed for this low-potential cytochrome, including a redox factor in cyclic photosynthetic electron transfer around PS I [235], a ferredoxin electron acceptor for removal of excess electrons under anaerobic conditions [236] and a factor involved in hydrogen metabolism [237]. Because, as discussed above, there is overwhelming evidence that PsbV is associated with PS II function, the negative $E_{m}$ value is a functional mystery, as participation in electron transfer reactions in the vicinity of the PS II oxygen-evolving complex would require a much more positive $E_{m}$ value.

Numerous studies have been directed at determining the factors that govern the $E_{m}$ properties of PsbV. Structural studies and analyses of the heme environment in soluble PsbV suggest that the heme environment of PsbV is quite similar to other c-type cytochromes with much higher $E_{m}$ values [223-225,238]. The $E_{m}$ of soluble PsbV is also $\mathrm{pH}$-dependent, possibly due to the protonation state of a tyrosine residue (probably ${ }^{82} \mathrm{Y}, \mathrm{T}$. elongatus numbering) [239] or the heme propionate group near ${ }^{49} \mathrm{~N}$ (T. elongatus numbering) [240]. Notably, mutation of ${ }^{49} \mathrm{~N}$ in Synechocystis $6803 \mathrm{PsbV}$ resulted in a small potential increase of $12-20 \mathrm{mV}$, but the $\mathrm{pH}$ dependence of the $E_{m}$ value for this mutant was not reported [241].

More recent studies have focused on determining the $E_{m}$ of PsbV bound to PS II. Because greater solvent exposure of the heme group tends to lower the $E_{m}$ of cytochromes, the $E_{m}$ of PsbV in the context of PS II may be different from that of the soluble protein. Indeed, several groups have reported slightly higher $E_{m}$ values for PsbV ( -110 or $-80 \mathrm{mV}$ ) in the context of PS II $[230,239,240]$. Furthermore, the $E_{m}$ of the PS II-bound form is pH-independent [239]. Kirilovsky and coworkers [230] characterized the $E_{m}$ of mutants containing sitedirected mutations of ${ }^{92} \mathrm{H}$, the sixth axial ligand to the heme in T. elongatus. The H92M and H92C mutants displayed lower $E_{m}$ values ( $-140 \mathrm{mV}$ and -164 , respectively) relative to wild type in the PS II-bound form $(-110 \mathrm{mV})$. The proteins carrying the H92M and H92C substitutions were more readily lost during PS II purification relative to native PsbV, but H92M and H92C mutants did not display any PS II activity defects at the cellular level. Based on these results, the $E_{m}$ of PsbV is not critical for PS II function under the conditions analyzed to date.

Recently, Guerrero and co-workers have reported an $E_{m}$ for PS II-bound PsbV of $+200 \mathrm{mV}$ [242]. This vastly different $E_{m}$ value was determined by reductive titration in the absence of the redox mediators typically used in such titrations. While a much lower $E_{m}$ value $(-200 \mathrm{mV})$ was observed for oxidative titration in the absence of redox mediators, the authors showed that treatment with dithionite, which is used to fully reduce PsbV prior to oxidative titration measurements, resulted in reduction of the $\mathrm{Mn}_{4} \mathrm{CaO}_{5}$ cluster and release of PsbV. Therefore, the authors postulate that the presence of redox mediators used in the titrations of previous reports partially reduce the $\mathrm{Mn}_{4} \mathrm{CaO}_{5}$ cluster of PS II, causing conformational changes which weaken the association of PsbV and lower its midpoint potential. If the $E_{m}$ of PsbV is $+200 \mathrm{mV}$ when bound to PS II, it is possible that electron transfer reactions (ms-s timescale) could occur between the 
heme group and the nearest redox-active cofactor, the $\mathrm{Mn}_{4} \mathrm{CaO}_{5}$ cluster ( $23 \AA$ ) [242]. To date, there is no experimental evidence for electron transfer between the heme group of PsbV and the $\mathrm{Mn}_{4} \mathrm{CaO}_{5}$ cluster, but these findings will surely stimulate future studies along these lines.

\section{CyanoQ}

\subsection{Structure}

The association of a PsbQ orthologue in cyanobacterial PS II was discovered by Pakrasi and co-workers when analyzing the complete protein complement of isolated PS II complexes from Synechcocystis 6803 [243]. The cyanobacterial protein, CyanoQ is related to the authentic PsbQ-1 and PsbQ-2 proteins from Arabidopsis (At4g21280 and At4g05180, respectively) rather than the more divergent members of the PsbQ gene family that have auxiliary functions [18]. The X-ray crystal structure of CyanoQ (Fig. 4B) is composed of a fourhelix bundle arranged in an up-down-up-down fold that resembles the PsbQ structure derived from spinach, despite retaining only $17 \%$ sequence identity [126-128]. However, the two proteins differ at their N-termini where, in the case of spinach PsbQ there are two parallel $\beta$-strands that are required for binding to the photosystem $[127,135]$. The absence of this structural motif in CyanoQ suggests the mechanism for binding to cyanobacterial PS II is different from the binding mechanism of PsbQ to plant PS II. In addition, comparison of the electrostatic surfaces of the two proteins reveals CyanoQ possesses a dipole with a positively charged surface comprising helices 2 and 3 and a negatively charged face formed by helices 3 and 4; in contrast, PsbQ has only a positively charged surface formed by helices 2 and 3 [128]. The corresponding helices of the two proteins also differ in length; notably, helix 2 of CyanoQ is split to give a kink by a fully conserved dipeptide composed of ${ }^{77} \mathrm{G}$ and ${ }^{78} \mathrm{P}$ in Synechocystis 6803.

A further unique feature of CyanoQ is a motif for a signal-peptide cleavage site with a lipid-modified cysteinyl residue, ${ }^{22} \mathrm{C}$. $[13,244]$. The equivalent lipidated cysteinyl is conserved in virtually all cyanobacteria. However, CyanoQ is not present in Gloeobacter violaceus or marine Prochloroccocus strains [13,20], and some strains of Synechococcus either lack a lipidation site or posses a modified lipobox [245]. Furthermore, there is no lipobox associated with the PsbQ-like proteins found in red and brown algae [128].

Both PsbQ and CyanoQ bind zinc; however, although both proteins crystallized with two bound zinc atoms, the binding sites were at different locations in the spinach and cyanobacterial structures. While PsbQ required the presence of zinc for crystallization, the structure of CyanoQ was solved both in the presence and absence of zinc [128]. In the CyanoQ structure, one of the zinc atoms was coordinated by ${ }^{76} \mathrm{H}$ and ${ }^{116} \mathrm{D}$, which are located above a cavity on the molecular face between helices 2 and 3 , and the size of the cavity increased in volume from $208 \AA^{3}$ to $249 \AA^{3}$ with zinc bound. Moreover, ${ }^{76} \mathrm{H}$ and ${ }^{116} \mathrm{D}$ and the majority of residues that make up the cavity are highly conserved. Therefore this feature might reflect a specific cation-mediated binding site and mechanism of interaction with another PS II subunit $[128,245]$. In contrast, the second zinc-binding site in the CyanoQ structure appears to be a crystallization artifact, as the ligands are not conserved.

Direct evidence for the binding of CyanoQ to PS II was obtained by isolating PS II complexes using a histidine tag placed on the C-terminus of the protein [12]. The isolation of PS II complexes has routinely been achieved by placing a histidine tag on one of the core antenna chlorophyll a-binding proteins, CP47 or CP43 [246,247]. The PS II complexes isolated using histidine-tagged CyanoQ were found to have a higher rate of oxygen evolution compared to PS II complexes isolated with the histidine tag on CP47. It has been suggested that this arises because the population of PS II isolated when the tag is on CP47 will include some inactive PS II centers that are undergoing assembly and repair [12,248].

The PS II crystal structures from T. elongatus and T. vulcanus do not contain bound CyanoQ [5,7,98]. However, to investigate the binding of CyanoQ to PS II, in silico docking studies have been undertaken using the T. elongatus 3.0-Å resolution structure (PDB ID: 2AXT), positioning CyanoQ next to PsbV and below CP43 [245].

The result of in silico docking studies of CyanoQ to the 1.9- $\AA$ resolution structure from $T$. vulcanus (PDB ID: 3ARC) is presented in Fig. 7 and was carried out as described in Fagerlund and Eaton-Rye [245]. The CyanoQ structure from T. elongatus was used to model the CyanoQ protein, as no sequence information is available for CyanoQ from T. vulcanus. The proximity of CyanoQ to the mouth of the putative B1 oxygen exit channel proposed by Gabdulkhakov et al. [111] is evident. In Fig. 7 CyanoQ sits immediately below the exit of the channel with its putative cation-mediated binding site facing PsbV.

\subsection{Function}

Synechocystis cells lacking CyanoQ have been shown to exhibit reduced oxygen-evolving activity compared to wild type under $\mathrm{CaCl}_{2}$ depleted conditions [13], but photoautotrophic growth of $\Delta c y a n o Q$ cells under either calcium-limiting or chloride-limiting conditions was only slightly, or not at all, retarded compared to wild type [13,39]. However, upon limitation of calcium, chloride and iron from the growth medium, $\Delta$ cyanoQ cells could not grow photoautotrophically [39]. Thus, CyanoQ is not absolutely required for optimal PS II activity unless additional stress conditions exist, a characteristic common to the cyanobacterial PS II extrinsic proteins.

The physiological role of CyanoQ has also been investigated through the phenotypic analysis of double mutants lacking other PS II extrinsic proteins [39]. Under calcium-limiting conditions, the photoautotrophic doubling time of the $\Delta p s b U: \Delta c y a n o Q$ mutant was extended by $30 \mathrm{~h}$ compared with $\Delta p s b U$ cells, whereas, photoautotrophic growth of the $\Delta p s b U: \triangle c y a n o Q$ strain showed little change under chloride-limiting conditions. However, the photoautotrophic doubling time of the $\triangle p s b O: \triangle c y a n o Q$ mutant in chloride-limiting media was extended by $10 \mathrm{~h}$ compared with $\Delta p s b 0$ cells. In calciumlimiting conditions, strains lacking PsbO are not photoautotrophic. The double mutant lacking PsbV was not photoautotrophic in nutrientreplete conditions; however, the obligate photoheterotrophic phenotype of the $\Delta p s b V: \Delta c y a n o Q$ strain was restored to photoautotrophy by growing the cells at $\mathrm{pH} 10$ [39]. As noted elsewhere a similar $\mathrm{pH}$ dependence has been observed with the $\Delta p s b 0: \Delta p s b U$ double mutant $[201,202,249]$ although the molecular mechanism underlying this $\mathrm{pH}$ effect is currently not known.

The absence of CyanoQ has a more pronounced effect on PS II activity and stability at the level of isolated PS II complexes. PS II complexes from $\triangle$ cyanoQ had greater oxygen-evolving activity in the presence of $20 \mathrm{mM} \mathrm{CaCl}_{2}$ compared to $5 \mathrm{mM} \mathrm{CaCl}_{2}$, whereas the rates observed with PS II complexes isolated from wild type were similar at both $\mathrm{CaCl}_{2}$ concentrations $[13,40]$. Furthermore, PS II complexes isolated from cells lacking CyanoQ were found to contain lower levels of PsbV than PS II complexes isolated from wild type [12,40]. Removal of PsbV is known to destabilize PS II and introduce a dependence on calcium and chloride for photoautotrophy (see section on the role of the PsbV protein). Hence, while the absence of CyanoQ is associated with an enhanced requirement for calcium and chloride (particularly in the double mutants lacking either PsbO or PsbU and in isolated PS II complexes) the reduced level of PsbV in the absence of CyanoQ is sufficient to maintain PS II activity that is similar to that seen in wild type in BG-11 growth medium. Additionally, PS II complexes lacking CyanoQ are more sensitive to the reducing agent hydroxylamine $\left(\mathrm{NH}_{2} \mathrm{OH}\right)$ than wild type [40]. This implies that while the water oxidation machinery is largely protected from hydroxylamine in wild type, it appears to be partially 


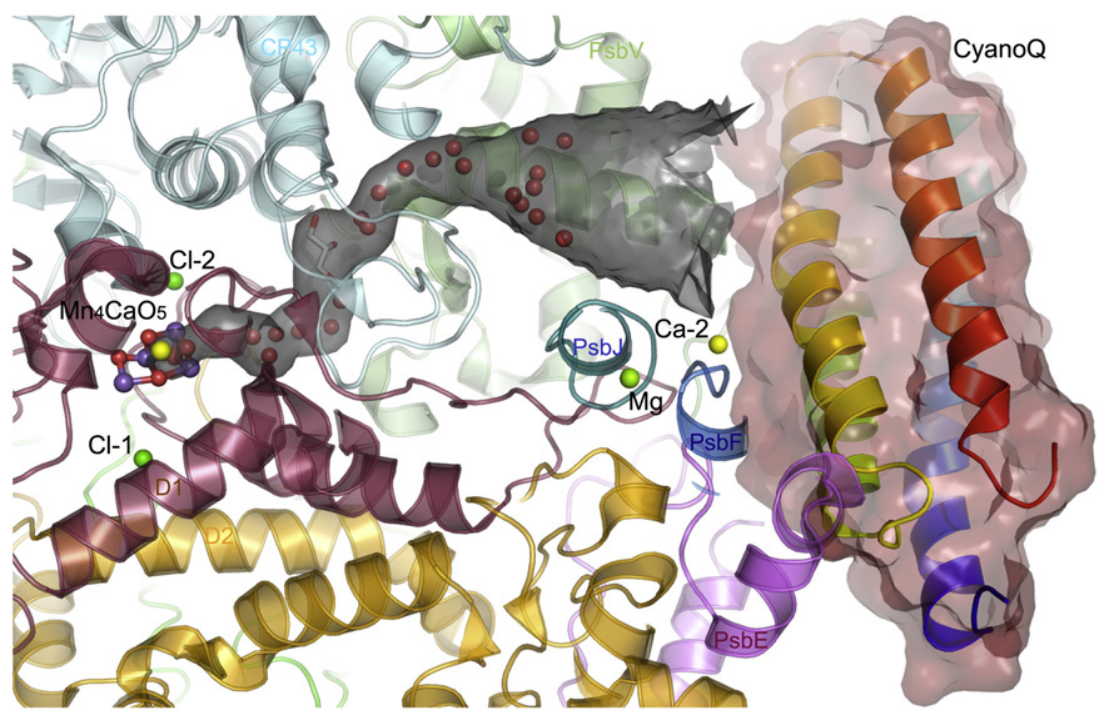

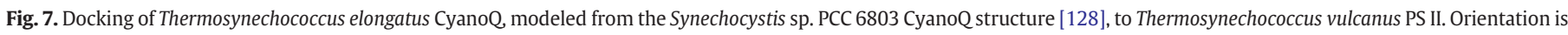

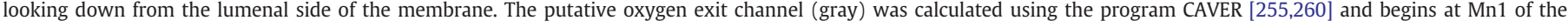

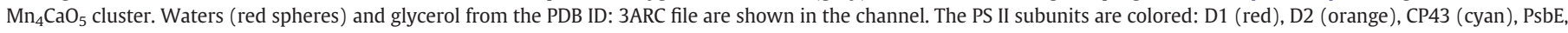

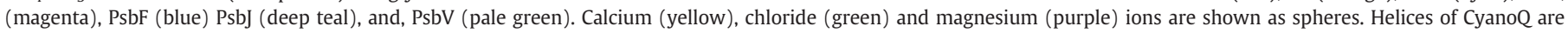
rainbow-colored from the $\mathrm{N}$-terminus (red).

exposed in the $\Delta$ cyanoQ mutant; consequently, the PS II complexes in this strain are more easily inactivated.

\section{CyanoP}

\subsection{Structure}

A PsbP orthologue was first identified in cyanobacteria in isolated PS II complexes from Synechocystis 6803 [243]. Subsequently, it has been shown through phylogenetic studies that PsbP-like proteins are found in all oxygenic photosynthetic organisms [18,250,251]. These PsbP-like proteins represent an extended family with eleven homologues identified in Arabidopsis belonging to three distinct groups: PS II-specific or authentic PsbP (two homologues), PsbP-like (two homologues), and those containing a "PsbP-like domain" (seven homologues) [123,251]. The homologue in Synechocystis 6803, referred to as CyanoP $[123,248]$, is most closely related to the PsbPlike PPL1 protein of Arabidopsis that has been suggested to play a role in the repair of photodamaged PS II [252]. The relationship between the PsbP-like proteins and CyanoP has also been supported by phylogenetic studies [251].

CyanoP, like CyanoQ, possesses a lipobox for signal-peptide cleavage and lipidation at a Cys [13]. Lipidation of CyanoP has not yet been demonstrated, but CyanoP was not released from the thylakoid membrane by salt washes that release other PS II extrinsic proteins, consistent with lipid-mediated membrane attachment. Also consistent with lipidation, the N-terminus of the protein was blocked when sequenced by Edman degradation [14]. To date CyanoP has not been found in any X-ray-derived crystal structures of cyanobacterial PS II; however, a functional relationship with PS II has been inferred from the observation that CyanoP was not present in mutant strains of Synechocystis 6803 that lack PS II: hence it seems expression of CyanoP requires the presence of PS II [14].

The crystal structure of CyanoP from T. elongatus has been solved to $2.8 \AA$ and is an antiparallel $ß$-sheet sandwiched between $\alpha$-helices (Fig. 3B) (PDB ID: 2XB3, [123]). This structure is similar to the structures solved for the tobacco and spinach PsbP proteins, despite low $(\sim 20 \%)$ sequence identity $[120,121,123]$. CyanoP was crystallized in the presence of zinc and it was shown the protein coordinated five zinc ions. Seven distinct contacts were made with the metal ion as a result of the protein's crystallization symmetry. It is possible that some of these sites may be artifacts of the crystallization process; however, two are highly conserved and the binding of zinc at one of these two sites (zinc ion 4 in [123]) is at an equivalent position to the zinc ion located in the PsbP structure from spinach. Moreover, conserved residues from both of the conserved zinc-binding sites have been previously identified as potential ligands that may interact with manganese in tobacco PsbP [253] (however, see [118] for a critical analysis of this hypothesis). The physiological significance of a divalent metal-binding site on CyanoP is unknown, although conservation of coordinating residues across phyla suggests it may have an as of yet unidentified role.

The docking of CyanoP to the PS II structure from T. elongatus (PDB ID: 2AXT) has been modeled and positions CyanoP below D2 and away from the other extrinsic proteins. In addition, a putative interaction between the C-terminus of the $\alpha$-subunit of cytochrome $b_{559}$ (PsbE) and a cavity on the CyanoP surface was identified [245]. In silico docking to the T. vulcanus PS II structure (PDB ID: 3ARC) yielded similar results (Fig. 8). However, an additional interaction with a helix incorporating ${ }^{277} \mathrm{~S}$ to ${ }^{294} \mathrm{~S}$ of loop E of $\mathrm{CP} 47$ was predicted. A Synechocystis 6803 mutant, the CP47: $\Delta$ (K277-E283) strain, containing a 7 amino acid deletion in this region of loop E, has been reported to have impaired photoautotrophic growth and impaired oxygen evolution and reduced levels of assembled PS II centers [254]. Intriguingly, the CAVER program [255] predicted the presence of a proton exit channel leading directly from $Y_{D}\left({ }^{160} \mathrm{~T}\right.$ of $\left.\mathrm{D} 2\right)$ to the bulk phase at the docking site of CyanoP (Fig. 7). The required hydrogen bond network for this protein pathway may be provided by waters and the side chain of ${ }^{180} \mathrm{R}: \mathrm{D} 2$. This channel is composed of residues from CP47, D1, PsbV and PsbE and the mouth comprises residues from D1, PsbV, PsbE and D2.

\subsection{Function}

Synechocystis 6803 strains lacking CyanoP typically exhibited photoautotrophic growth rates in BG-11 that are similar to wild type; even under chloride- or calcium-limiting conditions only small effects on photoautotrophic growth have been observed [13,14,256]. However, $\Delta$ cyanoP cells grown in $\mathrm{CaCl}_{2}$-depleted medium were observed to exhibit a rate of oxygen evolution $63 \%$ of that found for the wild type grown under similar conditions [13]. Furthermore, oxygen evolution measurements of PS II complexes purified via a histidine tag on CP47 


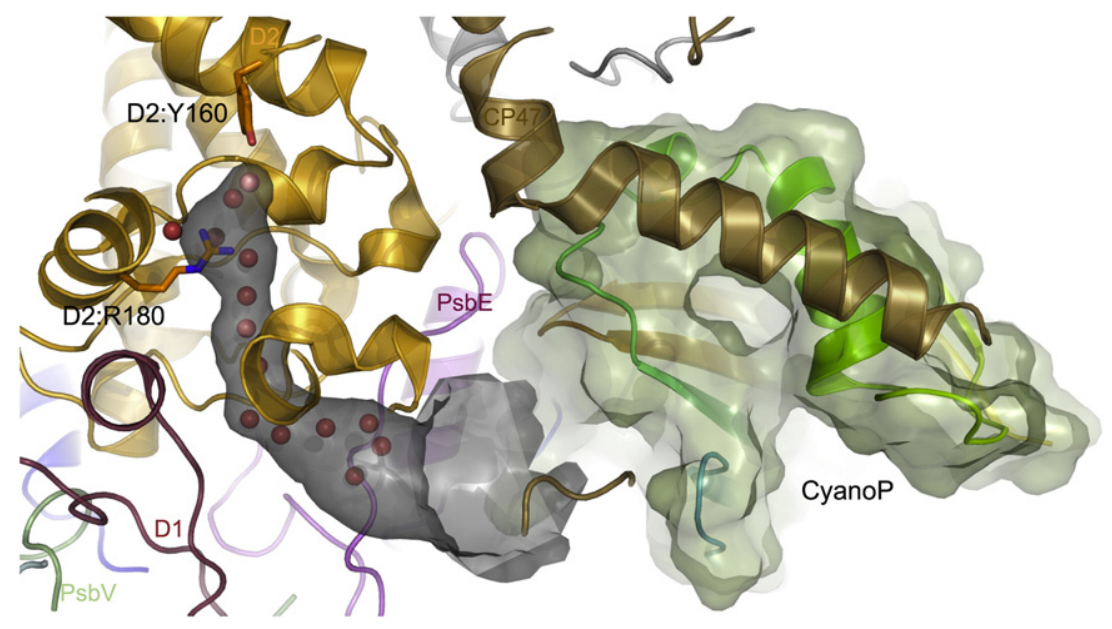

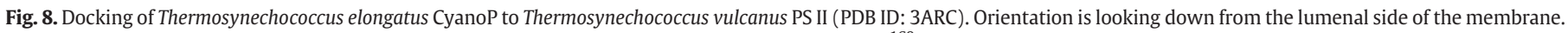

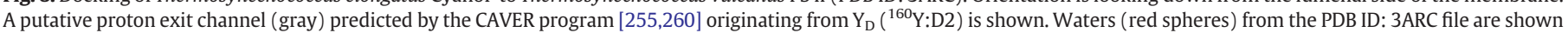

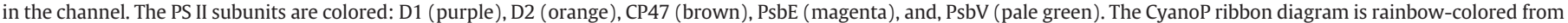
the N-terminus (red).

revealed that complexes isolated from $\triangle$ cyanoP cells had reduced oxygen-evolving activity compared to isolated PS II from wild type, particularly in the presence of reduced levels of calcium, suggesting CyanoP was necessary for optimal PS II activity in these preparations [13].

Since it has been suggested that PsbU and PsbV might have similar functions to PsbP and PsbQ in plants and green algae [199,257], the possibility that CyanoP is required when either PsbU or PsbV is absent was investigated by the construction of two double mutants, $\Delta p s b U$ : $\triangle c y a n o P$ and $\Delta p s b V: \Delta c y a n o P[256]$. The genetic deletion of CyanoP in combination with these other extrinsic components had little or no additional affects on photoautotrophic growth in normal as well as chloride- or calcium-limiting medium. A similar result was obtained with both the $\Delta p s b 0: \Delta c y a n o P$ and $\Delta c y a n o Q: \Delta c y a n o P$ strains. Likewise, no specific effects on oxygen evolution attributable to the removal of CyanoP were observed in any of the double mutants. Moreover, there was no additive effect of removing CyanoP in the $\Delta p s b 0: \Delta p s b U: \Delta c y a n o P$ triple mutant compared to the corresponding double mutant strains [256].

Since results obtained with mutants lacking CyanoP produced small effects and these results varied among different laboratories, a comparative study of the three available $\Delta$ cyanoP strains was carried out [258]. Competitive growth of the wild type with the mutants indicated a selective advantage for the presence of CyanoP. Furthermore, flash-induced oxygen yield experiments displayed a decreased level of oxygen produced on the third flash, increased level on the fourth flash, and an earlier dampening of the oscillations relative to the wild type in $\triangle c y a n o P$ cells. Additionally, thermoluminescence measurements demonstrated an apparent enhanced recombination between the $\mathrm{S}_{3}$ state of the oxygen-evolving complex and the reduced secondary plastoquinone electron acceptor $\mathrm{Q}_{\mathrm{B}}$ in the $\Delta$ cyanoP strains. These data indicate that the $\triangle$ cyanoP mutants exhibit a major $\mathrm{S}_{3} \mathrm{Q}_{\mathrm{B}}^{-}$ recombination in addition to $S_{2} Q_{B}^{-}$recombination, whereas the wild type predominately exhibits the thermoluminescence band corresponding to $\mathrm{S}_{2} \mathrm{Q}_{\mathrm{B}}^{-}$recombination. These results may implicate equilibration between $Y_{D}\left({ }^{160} Y\right.$ of D2) (or some other unspecified redoxactive species) and the $S_{3}$ state favoring, for example, $Y_{D}^{\text {red }} S_{3}$ over $\mathrm{Y}_{\mathrm{D}}^{\mathrm{ox}} \mathrm{S}_{2}$ in PS II centers lacking CyanoP, thereby pointing to a role for CyanoP in the stabilization of charge separation in PS II [258]. It is therefore of interest that a putative proton exit channel linking $Y_{D}$ to a possible docking site for CyanoP (Fig. 8) has been detected; however, the existence of this channel requires experimental verification.

Additionally, immunodetection of CyanoP in Synechocystis 6803 thylakoid membranes has indicated that CyanoP is present at a similar level to the PsbO protein [14], although this finding is controversial
[13]. Thus, oxygen flash yield experiments, thermoluminescence and western blotting suggest CyanoP is stoichiometrically present in PS II [14] in contrast to an earlier finding that it is associated only with 3\% of PS II complexes in the thylakoid membrane [13]. As detergent is required during the isolation of PS II complexes, it is possible that CyanoP is lost during complex purification [13,14,258]. However, despite the uncertainty regarding the stoichiometry of CyanoP, the role of this subunit is not essential under a wide range of conditions and it appears likely that the selective advantage conferred by the protein is linked to a regulatory role that remains to be fully elucidated.

\section{Conclusions}

As is evident in this review, over the last thirty years an enormous amount of effort has been focused on the elucidation of the structure and function of the extrinsic proteins of PS II. Much progress has clearly been made, but many questions remain unanswered. While phenomenologically the functions of these proteins have generally been identified, the mechanisms by which they influence PS II oxygen evolution remains elusive. While the structure of cyanobacterial PS II progresses towards atomic resolution (i.e. $1.2 \AA$ ), the organization of the CyanoP and CyanoQ subunits within the photosystem remains unclear. Experimental investigations into the structural and possible regulatory roles played by the extrinsic components in the numerous channels present in PS II must also be forthcoming. Additionally, while structures for isolated higher plant PsbP and PsbQ are available, no progress is evident in the elucidation of the overall structure of the higher plant photosystem. Clearly, much work remains.

\section{Acknowledgements}

Funding was provided by a United States Department of Energy grant \# DE-FG02-98ER20310 to T.M.B. and L.K.F., from the USDA National Institute of Food and Agriculture Grant \# 2008-35318-04605 to J.L.R., and by a Marsden Grant \# 08-UOO-043 to J.J.E.-R.

\section{References}

[1] R.J. Debus, The manganese and calcium ions of photosynthetic oxygen evolution, Biochim. Biophys. Acta 1102 (1992) 269-352.

[2] R.D. Britt, Oxygen evolution, in: D. Ort, C.F. Yocum (Eds.), Oxygenic Photosynthesis: The Light Reactions, Advances in Photosyntheis, vol. 4, Kluwer Academic Publishers, Dordrecht, 1996, pp. 137-164. 
[3] D.R. Ort, C.F. Yocum, Energy transfer and energy transduction in photosynthesis: an overview, in: D.R. Ort, C.F. Yocum (Eds.), Oxygenic Photosynthesis: The Light Reactions, Advances in Photosynthesis, vol. 4, Kluwer Academic Publishers, Dordrecht, 1996, pp. 1-9.

[4] G. Renger, Light induced oxidative water splitting in photosynthesis: energetics, kinetics and mechanism, Photochem. Photobiol. B: Biol. 104 (2011) 35-43.

[5] Y. Umena, K. Kawakami, J.-R. Shen, N. Kamiya, Crystal structure of oxygenevolving Photosystem II at a resolution of $1.9 \AA$ A, Nature 473 (2011) 55-60.

[6] C. Rosenberg, J. Christian, T.M. Bricker, C. Putnam-Evans, Site-directed mutagenesis of glutamate residues in the large extrinsic loop of the Photosystem II protein CP 43 affects oxygen-evolving activity and PS II assembly, Biochemistry 38 (1999) 15994-16000.

[7] K.N. Ferreira, T.M. Iverson, K. Maghlaoui, J. Barber, S. Iwata, Architecture of the photosynthetic oxygen-evolving center, Science 303 (2004) 1831-1838.

[8] A. Guskov, J. Kern, A. Gabdulkhakov, M. Broser, A. Zouni, W. Saenger, Cyanobacterial Photosystem II at 2.9-Å resolution and the roles of quinones, lipids, channels and chloride, Nat. Struct. Mol. Biol. 16 (2009) 334-342.

[9] M. Miyao, N. Murata, Role of the $33 \mathrm{kDa}$ polypeptide in preserving $\mathrm{Mn}$ in photosynthetic oxygen-evolution, FEBS Lett. 170 (1984) 350-354.

[10] R.L. Burnap, L.A. Sherman, Deletion mutagenesis in Synechocystis sp. PCC 6803 indicates that the Mn-stabilizing protein of Photosystem II is not essential for oxygen evolution, Biochemistry 30 (1991) 440-446.

[11] T.M. Bricker, Oxygen evolution in the absence of the $33 \mathrm{kDa}$ manganesestabilizing protein, Biochemistry 31 (1992) 4623-4628.

[12] J.L. Roose, Y. Kashino, H.B. Pakrasi, The PsbQ protein defines cyanobacterial Photosystem II complexes with highest activity and stability, Proc. Natl. Acad. Sci. U.S.A. 104 (2007) 2548-2553.

[13] L. Thornton, H. Ohkawa, J. Roose, Y. Kashino, N. Keren, H. Pakrasi, Homologs of plant PsbP and PsbQ proteins are necessary for regulation of Photosystem Il activity in the cyanobacterium Synechocystis 6803, Plant Cell 16 (2004) 2164-2175.

[14] Y. Ishikawa, W.P. Schroder, C. Funk, Functional analysis of the PsbP-like protein (sll1418) in Synechocystis sp. PCC 6803, Photosynth. Res. 84 (2005) 257-262.

[15] I. Enami, S. Kikuchi, T. Fukuda, H. Ohta, J.-R. Shen, Binding and functional properties of four extrinsic proteins of Photosystem II from a red alga, Cyanidium caldarium, as studied by release-reconstitution experiments, Biochemistry 37 (1998) 2787-2793.

[16] H. Ohta, T. Suzuki, M. Ueno, A. Okumura, S. Yoshihara, J.-R. Shen, I. Enami, Extrinsic proteins of Photosystem II: an intermediate member of the PsbQ protein family in red algal PS II, Eur. J. Biochem. 270 (2003) 4156-4163.

[17] K. Ifuku, S. Ishihara, R. Shimamoto, K. Ido, F. Sato, Structure, function, and evolution of the PsbP protein family in higher plants, Photosynth. Res. 98 (2008) 427-437.

[18] K. Ifuku, S. Ishihara, F. Satoh, Molecular functions of oxygen-evolving complex family proteins in photosynthetic electron flow, J. Integr. Plant Biol. 52 (2010) 723-734.

[19] K. Ifuku, K. Ido, F. Sato, Molecular functions of PsbP and PsbQ proteins in the Photosystem II supercomplex, J. Photochem. Photobiol. B: Biol. 104 (2011) 158-164.

[20] J. De Las Rivas, J. Barber, Analysis of the structure of the PsbO protein and its implications, Photosynth. Res. 81 (2004) 329-343.

[21] T.M. Bricker, R.L. Burnap, The extrinsic proteins of Photosystem II, in: T. Wydrzynski, K. Satoh (Eds.), Photosystem II: The Water/Plastoquinone OxidoReductase of Photosynthesis, Advances in Photosynthesis and Respiration, vol. 22, Springer, Dordrecht, 2005, pp. 95-120.

[22] Q. Xu, J. Nelson, T.M. Bricker, Secondary structure of the $33 \mathrm{kDa}$, extrinsic protein of Photosystem II: a far-UV circular dichroism study, Biochim. Biophys. Acta 1188 (1994) 427-431.

[23] J. De Las Rivas, J. Barber, Structure and thermal stability of Photosystem Il reaction centers studied by infrared spectroscopy, Biochemistry 36 (1997) 8897-8903.

[24] I.Z. Zubrzycki, L.K. Frankel, P.S. Russo, T.M. Bricker, Hydrodynamic studies on the manganese-stabilizing protein of Photosystem II, Biochemistry 37 (1998) 13553-13558

[25] B. Svensson, D.M. Tiede, B.A. Barry, Small-angle X-ray scattering studies of the manganese-stabilizing protein in Photosystem II, J. Phys. Chem. B: 106 (2002) 8485-8488.

[26] S.J. Darnell, D. Page, J.C. Mitchell, Automated decision-tree approach to predicting protein-protein interaction hot spots, Proteins 68 (2007) 813-823.

[27] J.P. Gallivan, D.A. Dougherty, Cation- $\pi$ interactions in structural biology, Proc. Natl. Acad. Sci. U.S.A. 96 (1999) 9459-9464.

[28] C. Putnam-Evans, J. Wu, R. Burnap, J. Whitmarsh, T.M. Bricker, Site-directed mutagenesis of the CP 47 Protein of Photosystem II: Alteration of conserved charged residues in the domain ${ }^{364} \mathrm{E}-{ }^{444} \mathrm{R}$, Biochemistry 35 (1996) 4046-4053.

[29] C. Putnam-Evans, T.M. Bricker, Site-directed mutagenesis of the CPa-1 protein of Photosystem II: Alteration of the basic residue pair ${ }^{384,385} \mathrm{R}$ to ${ }^{384,385} \mathrm{G}$ leads to a defect associated with the oxygen-evolving complex, Biochemistry 31 (1992) $11482-11488$

[30] M. Qian, S. Al-Khaldi, C. Putnam-Evans, T.M. Bricker, R.L. Burnap, Photoassembly of the Photosystem II $(\mathrm{Mn})_{4}$ cluster in site-directed mutants impaired in the binding of the manganese stabilizing protein, Biochemistry 36 (1997) 15244-15252.

[31] F.K. Bentley, J. Eaton-Rye, The effect of removing Photosystem II extrinsic proteins on dimer formation and recovery from photodamage in Synechocystis sp. PCC 6803, in: J.F. Allen, E. Gantt, J.H. Golbeck, B. Osmond (Eds.),
Photosynthesis. Energy from the Sun: 14th International Congress on Photosynthesis, Springer, Dordrecht, 2008, pp. 715-717.

[32] M.G. Kuhn, W.F.J. Vermaas, Deletion mutations in a long hydrophilic loop in the Photosystem II chlorophyll-binding protein CP43 in the cyanobacterium Synechocystis sp. PCC 6803, Plant Mol. Biol. 23 (1993) 123-133.

[33] J.J. Eaton-Rye, N. Murata, Evidence that the amino-terminus of the $33 \mathrm{kDa}$ extrinsic protein is required for binding to the Photosystem II complex, Biochim. Biophys. Acta 977 (1989) 219-226.

[34] H. Popelkova, M.M. Im, C.F. Yocum, N-terminal truncations of the manganesestabilizing protein identify two amino acid sequences required for binding of the eukaryotic protein to Photosystem II and reveal the absence of one bindingrelated sequence in cyanobacteria, Biochemistry 41 (2002) 10038-10045.

[35] A. Seidler, A.W. Rutherford, H. Michel, On the role of the N-terminus of the extrinsic 33 kDa protein in Photosystem II, Plant Mol. Biol. 31 (1996) 183-188.

[36] A. Motoki, M. Usui, T. Shimazu, M. Hirano, S. Katoh, A domain of the Mnstabilizing protein from Synechococcus elongatus involved in functional binding to Photosystem II, J. Biol. Chem. 277 (2002) 14747-14756.

[37] Z. Li, R.L. Burnap, Mutations of basic arginine residue 334 in the D1 protein of Photosystem II lead to unusual $\mathrm{S}_{2}$ state properties in Synechocystis sp. PCC 6803, Photosynth. Res. 72 (2002) 191-201.

[38] S.F. Al-Khaldi, J. Coker, J.R. Shen, R.L. Burnap, Characterization of site-directed mutants in manganese-stabilizing protein (MSP) of Synechocystis sp. PCC6803 unable to grow photoautotrophically in the absence of cytochrome c-550, Plant Mol. Biol. 43 (2000) 33-41.

[39] T.C. Summerfield, J.A. Shand, F.K. Bentley, J.J. Eaton-Rye, PsbQ (Sll1638) in Synechocystis sp. PCC 6803 is required for Photosystem II activity in specific mutants and in nutrient-limiting conditions, Biochemistry 44 (2005) 805-815.

[40] Y. Kashino, N. Inoue-Kashino, J. Roose, H. Pakrasi, Absence of the PsbQ protein results in destabilization of the PsbV protein and decreased oxygen evolution activity in cyanobacterial Photosystem II, J. Biol. Chem. 281 (2006) 20834-20841.

[41] T.M. Bricker, W.R. Odom, C.B. Queirolo, Close association of the $33 \mathrm{kDa}$ extrinsic protein with the apoprotein of CPa-1 in Photosystem II, FEBS Lett. 231 (1988) 111-117.

[42] I. Enami, T. Miyaoka, Y. Mochizuki, J.-R. Shen, K. Satoh, S. Katoh, Nearest neighbor relationships among constituent proteins of oxygen-evolving Photosystem Il membranes: binding and function of the extrinsic $33 \mathrm{kDa}$ protein, Biochim. Biophys. Acta 973 (1989) 35-40.

[43] R. Nagao, T. Suzuki, A. Okumura, A. Niikura, M. Iwai, N. Dohmae, T. Tomo, J.-R Shen, M. Ikeuchi, I. Enami, Topological analysis of the extrinsic PsbO, PsbP and PsbQ proteins in a green algal PSII complex by cross-linking with a water-soluble carbodiimide, Plant Cell Physiol. 51 (2010) 718-727.

[44] K.-C. Han, J.-R. Shen, M. Ikeuchi, Y. Inoue, Chemical crosslinking studies of extrinsic proteins in cyanobacterial Photosystem II, FEBS Lett. 355 (1994) $121-124$

[45] C.S. Hackett, P. Strittmatter, Covalent crosslinking of the active sites of vesiclebound cytochrome $b_{5}$ and NADH cytochrome $b_{5}$ reductase, J. Biol. Chem. 259 (1984) 3275-3282.

[46] W. Odom, T.M. Bricker, Interaction of CPa-1 with the manganese-stabilizing protein of Photosystem II: identification of domains cross-linked by 1-ethyl-3[3-(dimethylamino)propyl]carbodiimide, Biochemistry 31 (1992) 5616-5620.

[47] L.K. Frankel, T.M. Bricker, Interaction of $\mathrm{CPa}-1$ with the manganese-stabilizing protein of Photosystem II: identification of domains on CPa- 1 which are shielded from $\mathrm{N}$-hydroxysuccinimide biotinylation by the manganese-stabilizing protein, Biochemistry 31 (1992) 11059-11063.

[48] I. Enami, S. Ohta, S. Mitsuhashi, S. Takahashi, M. Ikeuchi, S. Katoh, Evidence from crosslinking for a close association of the extrinsic $33 \mathrm{kDa}$ protein with the $9.4 \mathrm{kDa}$ subunit of cytochrome $\mathrm{b}_{559}$ and the $4.8 \mathrm{kDa}$ product of the psbI gene in oxygen-evolving Photosystem II complexes from spinach, Plant Cell Physiol. 33 (1992) 291-297.

[49] G.E. Means, R.E. Feeney, Chemical Modification of Proteins, Holden-Day, Inc., San Francisco, 1971.

[50] J. Nield, J. Barber, Refinement of the structural model for the Photosystem II supercomplex of higher plants, Biochim. Biophys. Acta 1757 (2006) 353-361.

[51] D. Slowik, M. Rossmann, P.V. Konarev, K.-D. Irrgang, W. Saenger, Structura investigation of PsbO from plant and cyanobacterial Photosystem II, J. Mol. Biol. 407 (2011) 125-137.

[52] T.M. Bricker, L.K. Frankel, The structure and function of the $33 \mathrm{kDa}$ extrinsic protein of Photosystem II. A critical review, Photosynth. Res. 56 (1998) 157-173.

[53] H. Popelkova, C.F. Yocum, PsbO, the manganese-stabilizing protein: analysis of the structure-function relations that provide insights into its role in Photosystem II, Photochem. Photobiol. B: Biol. 104 (2011) 179-190.

[54] Q. Xu, T.M. Bricker, Structural organization of proteins on the oxidizing side of Photosystem II. Two molecules of the 33-kDa manganese-stabilizing protein per reaction center, J. Biol. Chem. 267 (1992) 25816-25821.

[55] C. Leuschner, T.M. Bricker, Interaction of the $33 \mathrm{kDa}$ extrinsic protein with Photosystem II: rebinding of the $33 \mathrm{kDa}$ extrinsic protein to Photosystem Il membranes which contain four, two, or zero manganese per Photosystem Il reaction center, Biochemistry 35 (1996) 4551-4557.

[56] Y. Yamamoto, S. Nakayama, C.L. Cohn, D.W. Krogmann, Highly efficient purification of the 33-, 24-, and 18-kDa proteins in spinach Photosystem II by butanol/water phase partitioning and high-performance liquid chromatography, Arch. Biochem. Biophys. 255 (1987) 156-161.

[57] A. Seidler, Introduction of a histidine tail at the N-terminus of a secretory protein expressed in E. coli, Protein Eng. 7 (1994) 1277-1280. 
[58] S.D. Betts, J.R. Ross, E. Pichersky, C.F. Yocum, Mutation Val235Ala weakens binding of the 33-kDa manganese-stabilizing protein of Photosystem II to one of two sites, Biochemistry 36 (1997) 4047-4053.

[59] R. Murakami, K. Ifuku, A. Takabayashi, T. Shikanai, T. Endo, F. Sato, Functiona dissection of two Arabidopsis PsbO proteins Psb01 and PsbO2, FEBS J. 272 (2005) 2165-2175.

[60] H. Popelkova, M.M. Im, J. D'Auria, S.D. Betts, N. Lydakis-Simantiris, C.F. Yocum, Nterminus of the Photosystem II manganese-stabilizing protein: effects of sequence elongation and truncation, Biochemistry 41 (2002) 2702-2711.

[61] H. Popelkova, A. Commet, C.F. Yocum, Asp157 is required for the function of PsbO, the Photosystem II manganese-stabilizing protein, Biochemistry 48 (2009) 11920-11928.

[62] L.K. Frankel, J.A. Cruz, T.M. Bricker, The role of carboxylic acid residues on the manganese-stabilizing protein in its binding to Photosystem II, Biochemistry 38 (1999) 14271-14278.

[63] H. Popelkova, M.M. Im, C.F. Yocum, Binding of manganese-stabilizing protein to Photosystem II: identification of essential N-terminal threonine residues and domains that prevent nonspecific binding, Biochemistry 42 (2003) 6193-6200.

[64] H. Popelkova, A. Commet, T. Kuntzleman, C.F. Yocum, Inorganic cofactor stabilization and retention: the unique functions of the two PsbO subunits of eukaryotic Photosystem II, Biochemistry 47 (2008) 12600-12953.

[65] J.L. Roose, C.F. Yocum, H. Popelkova, Binding stoichiometry and affinity of the manganese-stabilizing protein affects redox reactions on the oxidizing side of Photosystem II, Biochemistry 50 (2011) 5988-5998.

[66] R. Anati, N. Adir, Crystallization of dimers of the manganese-stabilizing protein of Photosystem II, Photosynth. Res. 64 (2000) 167-177.

[67] E.J. Boekema, J.F.L. van Breeman, H. van Roon, J.P. Dekker, Conformational changes in Photosystem II supercomplexes upon removal of extrinsic subunits, Biochemistry 39 (2000) 12907-12915.

[68] L.K. Frankel, T.M. Bricker, Interaction of the $33 \mathrm{kDa}$ extrinsic protein with Photosystem II: identification of domains on the $33 \mathrm{kDa}$ protein that are shielded from NHS biotinylation by Photosystem II, Biochemistry 34 (1995) 7492-7497.

[69] H. Popelkova, A. Commet, C.F. Yocum, The double mutant DL6MW241F in PsbO, the Photosystem II manganese-stabilizing protein, yields insights into its evolution of its structure and function, FEBS Lett. 584 (2010) 4009-4014.

[70] T.-A. Ono, Y. Inoue, Mn-preserving extraction of 33-, 24- and 16-kDa proteins from $\mathrm{O}_{2}$-evolving PS II particles by divalent salt-washing, FEBS Lett. 164 (1983) $255-260$.

[71] T. Ono, Y. Inoue, S-State turnover in the $\mathrm{O}_{2}$-evolving system of $\mathrm{CaCl}_{2}$-washed Photosystem II particles depleted of three peripheral proteins as measured by thermoluminescence. Removal of $33 \mathrm{kDa}$ protein inhibits $S_{3}$ to $S_{4}$ transition, Biochim. Biophys. Acta 806 (1985) 331-340.

[72] M. Miyao, N. Murata, J. Lavorel, B. Maison-peteri, A. Boussac, A.-L. Etienne, Effect of the 33-kDa protein on the S-state transitions in photosynthetic oxygen evolution, Biochim. Biophys. Acta 890 (1987) 151-159.

[73] R. Murakami, K. Ifuku, A. Takabayashi, T. Shikanai, T. Endo, F. Sato, Characterization of an Arabidopsis thaliana mutant with impaired psbO, one of two genes encoding extrinsic 33-kDa proteins in Photosystem II, FEBS Lett. 523 (2002) 138-142.

[74] H. Liu, L.K. Frankel, T.M. Bricker, Functional analysis of Photosystem II in a PsbO-1 deficient mutant in Arabidopsis thaliana, Biochemistry 46 (2007) 7607-7613.

[75] Y. Allahverdiyeva, F. Mamedov, M. Holmström, M. Nurmi, B. Lundin, S. Styring, C. Spetea, E.-M. Aro, Comparison of the electron transport properties of the psbo1 and psbo2 mutants of Arabidopsis thaliana, Biochim. Biophys. Acta 1787 (2009) 1230-1237.

[76] R.L. Burnap, J.-R. Shen, P.A. Jursinic, Y. Inoue, L.A. Sherman, Oxygen yield and thermoluminescence characteristics of a cyanobacterium lacking the manganese-stabilizing protein of Photosystem II, Biochemistry 31 (1992) 7404-7410.

[77] I. Vass, K.M. Cool, D. Zsuzsanra, S.R. Mayes, J. Barber, Thermoluminescence and flash oxygen characterization of the IC2 deletion mutant of Synechocystis sp. PCC 6803 lacking the Photosystem II 33 kDa protein, Biochim. Biophys. Acta 1102 (1992) 195-201.

[78] M.R. Razeghifard, T. Wydrzynski, R.J. Pace, R.L. Burnap, $Y_{Z} \bullet$ reduction kinetics in the absence of the manganese-stabilizing protein of Photosystem II, Biochemistry 36 (1997) 14474-14478.

[79] K. Lindberg, L.-E. Andreasson, On the binding and function of chloride in Photosystem II, in: P. Mathis (Ed.), Photosynthesis: From Light to Biosphere: Proceedings of the Xth International Photosynthesis Congress, vol. 2, Kluwer Academic Publishers, Dordrecht, 1995, pp. 401-404.

[80] T. Ono, J.L. Zimmerman, Y. Inoue, A.W. Rutherford, EPR evidence for a modified Sstate transition in chloride-depleted Photosystem II, Biochim. Biophys. Acta 851 (1986) 193-201.

[81] S. Styring, M. Miyao, A.W. Rutherford, Formation and flash-dependent oscillation of the $\mathrm{S}_{2}$-state multiline EPR signal in an oxygen-evolving Photosystem II preparation lacking the three extrinsic proteins in the oxygen-evolving system, Biochim. Biophys. Acta 767 (1987) 32-38.

[82] H. Wincencjusz, H.J. van Gorkom, C.F. Yocum, The photosynthetic oxygen evolving complex requires chloride for its redox state $S_{2} \rightarrow S_{3}$ and $S_{3} \rightarrow S_{0}$ transitions but not for $S_{0} \rightarrow S_{1}$ or $S_{1} \rightarrow S_{2}$ transitions, Biochemistry 36 (1997) 3663-3670.

[83] K. Lubbers, W. Drevenstedt, W. Junge, Chloride-depletion of photosynthetic water oxidase: no proton release during the second oxidation step, $S_{2}^{*}-S^{*}$, and a transmembrane radical pair recombination from the third on, Biochim. Biophys. Acta 336 (1993) 304-308.
[84] T. Ono, T. Noguchi, Y. Inoue, M. Kusunoki, H. Yamaguchi, H. Oyanagi, XANES spectroscopy for monitoring intermediate reaction states of $\mathrm{Cl}^{-}$-depleted $\mathrm{Mn}$ cluster in photosynthetic water oxidation enzyme, J. Am. Chem. Soc. 117 (1995) 6386-6387.

[85] A. Boussac, J.L. Zimmermann, A.W. Rutherford, EPR signals from modified charge accumulation states of the oxygen evolving enzyme in calcium deficient Photosystem II, Biochemistry 28 (1989) 8984-8989.

[86] J.C. Depaula, P.M. Li, A.F. Miller, B.W. Wu, G.W. Brudwig, Effect of the 17kilodalton and 23-kilodalton polypeptides, calcium, and chloride on electrontransfer in Photosystem II, Biochemistry 25 (1986) 6487-6494.

87] M. Haumann, W. Junge, Evidence for impaired hydrogen-bonding of tyrosine $Y_{Z}$ in calcium-depleted Photosystem II, Biochim. Biophys. Acta 1411 (1999) 121-133.

[88] T.M. Bricker, L.K. Frankel, The Arabidopsis psbo1 mutant cannot efficiently utilize calcium in support of photosynthetic oxygen evolution, J. Biol. Chem. 283 (2008) 29022-29027.

[89] G. Mavankal, D.C. McCain, T.M. Bricker, Effects of chloride on paramagnetic coupling of manganese in calcium chloride-washed Photosystem II preparations, FEBS Lett. 202 (1986) 235-239.

[90] M. Miyao, N. Murata, Calcium ions can be substituted for the $24 \mathrm{kDa}$ polypeptide in photosynthetic oxygen evolution, FEBS Lett. 168 (1984) 118-120.

[91] T.A. Ono, Y. Inoue, Reconstitution of photosynthetic oxygen evolving activity by rebinding of $33 \mathrm{kDa}$ protein to $\mathrm{CaCl}_{2}$-extracted PS II particles, FEBS Lett. 166 (1984) 381-384

92] J. Cole, M. Boska, N.V. Blough, K. Sauer, Reversible and irreversible effects of alkaline $\mathrm{pH}$ on Photosystem II electron-transfer reactions, Biochim. Biophys. Acta 848 (1986) 41-47.

[93] W.J. Coleman, Govidjee, A model for the mechanism of chloride activation of oxygen evolution in Photosystem II, Photosynth. Res. 13 (1987) 199-223.

[94] R. Wales, B.J. Newman, D. Pappin, J.C. Gray, The extrinsic $33 \mathrm{kDa}$ protein of the oxygen-evolving complex of Photosystem II is a putative calcium-binding protein and is encoded by a multi-gene family in pea, Plant Mol. Biol. 12 (1989) 439-451.

[95] A.N. Webber, J.C. Gray, Detection of calcium binding by Photosystem II polypeptides immobilized onto nitrocellulose membranes, FEBS Lett. 249 (1989) 79-82.

[96] A. Seidler, The extrinsic proteins of Photosystem II, Biochim. Biophys. Acta 1277 (1996) 35-60.

[97] A. Seidler, A.W. Rutherford, The role of the extrinsic $33 \mathrm{kDa}$ protein in $\mathrm{Ca}^{+2}$ binding in Photosystem II, Biochemistry 35 (1996) 12104-12110.

[98] B. Loll, N. Kern, W. Saenger, A. Zouni, J. Biesiadka, Towards complete cofactor arrangement in the $3.0 \AA$ resolution structure of Photosystem II, Nature 438 (2006) 1040-1044.

[99] J.W. Murray, J. Barber, Identification of a calcium-binding site in the PsbO protein of Photosystem II, Biochemistry 45 (2006) 4128-4130.

[100] J.B. Philbrick, B.A. Diner, B.A. Zilinskas, Construction and characterization of cyanobacterial mutants lacking the manganese-stabilizing protein of Photosystem II, J. Biol. Chem. 266 (1991) 13370-13376

[101] D.H. Engels, A. Lott, G.H. Schmid, E.K. Pistorious, Inactivation of the wateroxidizing enzyme in manganese stabilizing protein-free cells of the cyanobacteria Synechococcus PCC 7942 and Synechocystis PCC 6803 during dark incubation and conditions leading to photoactivation, Photosynth. Res. 42 (1994) 227-244.

[102] S.P. Mayfield, P. Bennoun, J.D. Rochaix, Expression of the nuclear-encoded OEE1 protein is required for oxygen evolution and stability of Photosystem II particles in Chlamydomonas reinhardtii, EMBO J. 6 (1987) 313-318.

[103] X. Yi, M. McChargue, S.M. Laborde, L.K. Frankel, T.M. Bricker, The manganesestabilizing protein is required for Photosystem II assembly/stability and photoautotrophy in higher plants, J. Biol. Chem. 280 (2005) 16170-16174.

[104] R.S. Hutchinson, J.J. Steenhuis, C.F. Yocum, M.R. Razeghifard, B.B. Barry, Deprotination of the 33-kDa, extrinsic, manganese-stabilizing subunit accompanies photooxidation of the manganese in Photosystem II, J. Biol. Chem. 274 (1999) 31987-31995.

[105] S.K. Hong, S.A. Pawlikowski, K.A. VanderMeulen, C.F. Yocum, The oxidation state of the Photosystem II manganese cluster influences the structure of the manganese-stabilizing protein, Biochim. Biophys. Acta 1504 (2001) 262-274.

[106] H. Popelkova, S.D. Betts, N. Lydakis Simantiris, M.M. Im, E. Swenson, C.F. Yocum, Mutagenesis of basic residues R151 and R161 in manganese-stabilizing protein of Photosystem II causes inefficient binding of chloride to the oxygen-evolving complex, Biochemistry 45 (2006) 3107-3115.

[107] J.L. Roose, C.F. Yocum, H. Popelkova, Function of PsbO, the Photosystem II manganese-stabilizing protein: probing the role of aspartic acid 157, Biochemistry 49 (2010) 6042-6051.

[108] J.W. Murray, J. Barber, Structural characteristics of channels and pathways in Photosystem II including the identification of an oxygen channel, J. Struct. Biol. 159 (2007) 228-237.

[109] F.M. Ho, Uncovering channels in Photosystem II by computer modelling: current progress, future prospects, and lessons from analogous systems, Photosynth. Res. 98 (2008) 503-522.

[110] F.M. Ho, S. Styring, Access channels and methanol binding site to the $\mathrm{CaMn}_{4}$ cluster in Photosystem II based on solvent accessibility simulations, with implications for substrate water access, Biochim. Biophys. Acta 1777 (2008) 140-153.

[111] A. Gabdulkhakov, A. Guskov, M. Broser, J. Kern, F. Müh, W. Saenger, A. Zouni, Probing the accessibility of the $\mathrm{Mn}_{4} \mathrm{Ca}$ cluster in Photosystem II; channels 
calculation, noble gas derivatization, and cocrystallization with DMSO, Structure 17 (2009) 1223-1234.

[112] C. Spetea, T. Hundal, B. Lundin, M. Heddad, I. Adamska, B. Andersson, Multiple evidence for nucleotide metabolism in the chloroplast thylakoid lumen, Proc. Natl. Acad. Sci. U.S.A. 101 (2004) 1409-1414.

[113] B. Lundin, S. Thuswaldner, T. Shutova, S. Eshaghi, G. Samuelsson, J. Barber, B. Andersson, C. Spetea, Subsequent events to GTP binding by the plant PsbO protein: structural changes, GTP hydrolysis and dissociation from the Photosystem II complex, Biochim. Biophys. Acta 1767 (2007) 500-508.

[114] B. Lundin, M. Hansson, B. Schoefs, A.V. Vener, C. Spetea, The Arabidopsis PsbO2 protein regulates dephosphorylation and turnover of the Photosystem II reaction center D1 protein, Plant J. 49 (2007) 528-539.

[115] E.-M. Aro, I. Virgin, B. Andersson, Photoinhibition of Photosystem II. Inactivation, protein damage and turnover, Biochim. Biophys. Acta 1143 (1993) 113-134.

[116] E.-M. Aro, M. Suorsa, A. Rokka, Y. Allahverdiyeva, V. Paakkarinen, A. Saleem, N. Battchikova, E. Rintamäki, Dynamics of Photosystem II: a proteomic approach to thylakoid protein complexes, J. Exp. Bot. 56 (2005) 347-356.

[117] B. Lundin, M. Nurmi, M. Rojas-Stuetz, E.-M. Aro, I. Adamski, C. Spetea, Towards understanding the functional difference between the two PsbO isoforms in Arabidopsis thaliana-insights from phenotypic analyses of psbO knockout mutants, Photosynth. Res. 98 (2008) 405-414.

[118] T.M. Bricker, L.K. Frankel, Auxiliary functions of the PsbO, PsbP and PsbQ proteins of higher plant Photosystem II: a critical analysis, Photochem. Photobiol. B: Biol. 104 (2011) 165-178.

[119] T. Jansen, C. Rother, J. Steppuhn, H. Reinke, K. Beyreuther, C. Jansson, B. Andersson, R.G. Herrmann, Nucleotide sequence of cDNA clones encoding the complete $23 \mathrm{kDa}$ and $16 \mathrm{kDa}$ precursor proteins associated with the oxygenevolving complex from spinach, FEBS Lett. 216 (1987) 234-240.

[120] K. Ifuku, T. Nakatsu, H. Kato, F. Sato, Crystal structure of the PsbP protein of Photosystem II from Nicotiana tabacum, EMBO Rep. 5 (2004) 362-367.

[121] J. Kohoutova, I.K. Smatanova, J. Brynda, M. Lapkouski, J.L. Revuelta, J.B. Arellano, R. Ettrich, Crystallization and preliminary crystallographic characterization of the extrinsic PsbP protein of Photosystem II from Spinacia oleracea, Acta Crystallogr. F 65 (2009) 111-115.

[122] R. Ristvejova, M. Lapkouski, J. Kopecky, V.K. Hofbauerova, Z. Sofova, S. GonzalezPerez, I.K. Smatanova, J.L. Revuelta, J.B. Arellano, R. Ettrich, Structure of PsbP protein from Spinacea oleracea at $1.98 \AA$, doi:10.2210/pdb2vu4/pdb.

[123] F. Michoux, K. Takasaka, M. Boehm, P.J. Nixon, J.W. Murray, Structure of CyanoP at $2.8 \AA$ : implications for the evolution and function of the PsbP subunit of Photosystem II, Biochemistry 49 (2010) 7411-7413.

[124] M. Stewart, R.P. Baker, $1.9 \AA$ A resolution crystal structure of the Saccharomyces cerevisiae Ran-binding protein Mog1p, J. Mol. Biol. 299 (2000) 213-223.

[125] M. Oki, T. Nishimoto, A protein required for nuclear-protein import, Mog1p, directly interacts with GTP-Gsp1p, the Saccharomyces cerevisiae ran homologue, Proc. Natl. Acad. Sci. U.S.A. 95 (1998) 15388-15393.

[126] V. Calderone, M. Trabucco, A. Vujicic, R. Battistutta, G.M. Giacometti, F. Andreucci, R. Barbato, G. Zanotti, Crystal structure of the PsbQ protein of Photosystem II from higher plants, EMBO Rep. 4 (2003) 900-905.

[127] M. Balsera, J.B. Arellano, J.L. Revuelta, J. De Las Rivas, J.A. Hermoso, A $1.49 \AA$ resolution crystal structure of PsbQ from Photosystem II of Spinacia oleraced reveals a PPII structure in the N-terminal region, J. Mol. Biol. 350 (2005) 1051-1058.

[128] S.A. Jackson, R.D. Fagerlund, S.M. Wilbanks, J.J. Eaton-Rye, Crystal structure of PsbQ from Synechocystis sp. PCC 6803 at 1.8 A: implications for binding and function in cyanobacterial Photosystem II, Biochemistry 49 (2010) 2765-2767.

[129] J. Ristvejova, V. Kopecky Jr., Z. Sovova, M. Balsera, J.B. Arellano, M. Green, R. Ettrich, Structure and dynamics of the N-terminal loop of PsbQ from Photosystem II of Spinacia oleracea, Biochem. Biophys. Res. Commun. 345 (2006) 287-291.

[130] M. Miyao, N. Murata, The mode of binding of three extrinsic proteins of $33 \mathrm{kDa}$, $23 \mathrm{kDa}$ and $18 \mathrm{kDa}$ in the Photosystem II complex of spinach, Biochim. Biophys. Acta 977 (1989) 315-321.

[131] K. Kavelaki, D.F. Ghanotakis, Effects of the manganese complex on the binding of the extrinsic proteins (17, 23 and $33 \mathrm{kDa}$ ) of Photosystem II, Photosynth. Res. 29 (1991) 149-155.

[132] K. Ifuku, F. Sato, A truncated mutant of the extrinsic 23-kDa protein that absolutely requires the extrinsic $17-\mathrm{kDa}$ protein for $\mathrm{Ca}^{2+}$ retention in Photosystem II, Plant Cell Physiol. 43 (2002) 1244-1249.

[133] A. Tohri, N. Dohmae, T. Suzuki, H. Ohta, Y. Inoue, I. Enami, Identification of domains on the extrinsic $23 \mathrm{kDa}$ protein possibly involved in electrostatic interaction with the extrinsic $33 \mathrm{kDa}$ protein in spinach Photosystem II, Eur. J. Biochem. 271 (2004) 962-971.

[134] T.M. Bricker, L.K. Frankel, Carboxylate groups on the manganese-stabilizing protein are required for efficient binding of the $24 \mathrm{kDa}$ extrinsic protein to Photosystem II, Biochemistry 42 (2003) 2056-2061.

[135] T. Kuwabara, T. Murata, M. Miyao, N. Murata, Partial degradation of the 18kilodalton protein of the photosynthetic oxygen-evolving complex a study of a binding site, Biochim. Biophys. Acta 850 (1986) 146-155.

[136] G.D. Meades Jr., A. McLachlan, L. Sallans, P.A. Limbach, T.M. Bricker, Association of the $17 \mathrm{kDa}$ extrinsic protein with Photosystem II in higher plants, Biochemistry 44 (2005) 15216-15221.

[137] T. Suzuki, J. Minagawa, T. Tomo, K. Sonoike, H. Ohta, I. Enami, Binding and functional properties of the extrinsic proteins in oxygen-evolving Photosystem Il particles from a green alga, Chlamydomonas reinhardtii having his-tagged CP47, Plant Cell Physiol. 44 (2003) 76-84.
[138] T. Suzuki, O. Tada, M. Makimura, A. Tohri, H. Ohta, Y. Yamamoto, Isolation and characterization of oxygen-evolving Photosystem II complexes retaining the PsbO, P and Q proteins from Euglena gracilis, Plant Cell Physiol. 45 (2004) 1168-1175.

[139] Y. Yamamoto, F. Kubota, Specific release of the extrinsic 18-kDa protein from spinach Photosystem II particles by the treatment with $\mathrm{NaCl}$ and methanol and its application for large scale purification of the three extrinsic proteins of Photosystem II without chromatography, Biochim. Biophys. Acta 893 (1987) 579-583.

[140] M. Bernier, R. Carpentier, The action of mercury on the binding of the extrinsic polypeptides associated with the water oxidizing complex of Photosystem II, FEBS Lett. 360 (1995) 251-254.

[141] H. Yu, X. Xu, R. Britt, The $33 \mathrm{kDa}$ protein can be removed without affecting the association of the 23 and $17 \mathrm{kDa}$ proteins with the lumenal side of PS II of spinach, Biochemistry 45 (2006) 3404-3411.

[142] S. Caffarri, R. Kouril, S. Kereiche, E.J. Boekema, R. Croce, Functional architecture of higher plant Photosystem II supercomplexes, EMBO J. 28 (2009) 3052-3063.

[143] H. Popelkova, C.F. Yocum, Current status of the role of $\mathrm{Cl}^{-}$ion in the oxygenevolving complex, Photosynth. Res. 93 (2007) 111-121.

[144] M. Miqyass, H.J. van Gorkom, C.F. Yocum, The PS II calcium site revisited, Photosynth. Res. 92 (2007) 275-287.

[145] H.-E. Akerlund, C. Jannson, B. Andersson, Reconstitution of oxygen evolution in high salt washed Photosystem II particles, Biochim. Biophys. Acta 681 (1982) 1-10.

[146] M. Miyao, N. Murata, Partial disintegration and reconstitution of the photosynthetic oxygen evolution complex, Biochim. Biophys. Acta 725 (1983) 87-93.

[147] M. Miyao, N. Murata, The $\mathrm{Cl}^{-}$effect on photosynthetic oxygen evolution: interaction of $\mathrm{Cl}^{-}$with $18-\mathrm{kDa}, 24-\mathrm{kDa}$ and 33-kDa proteins, FEBS Lett. 180 (1985) 303-308.

[148] D.F. Ghanotakis, G.T. Babcock, C.F. Yocum, Calcium reconstitutes high rates of oxygen evolution in polypeptide depleted Photosystem II preparations, FEBS Lett. 167 (1984) 127-130.

[149] D.F. Ghanotakis, G.T. Babcock, C.F. Yocum, On the role of water-soluble polypeptides $(17,23 \mathrm{kDa})$ calcium and chloride in photosynthetic oxygen evolution, FEBS Lett. 192 (1985) 1-3.

[150] H.Y. Nakatani, Photosynthetic oxygen evolution does not require the participation of polypeptides of 16 and $24 \mathrm{kDa}$, Biochem. Biophys. Res. Commun. 120 (1984) 299-304.

[151] A. Boussac, A.W. Rutherford, $\mathrm{Ca}^{2+}$ binding to the oxygen evolving enzyme varies with the redox state of the Mn enzyme, Biochim. Biophys. Acta 236 (1988) 432-436.

[152] P. Adelroth, K. Lindberg, L.-E. Andreasson, Studies of $\mathrm{Ca}^{+2}$ binding in spinach Photosystem II using ${ }^{45} \mathrm{Ca}^{+2}$, Biochemistry 34 (1995) 9021-9027.

[153] H. Wincencjusz, C.F. Yocum, H.J. van Gorkom, S-state dependence of chloride binding affinities and exchange dynamics in the intact and polypeptide depleted $\mathrm{O}_{2}$ evolving complex of Photosystem II, Biochemistry 37 (1998) 8595-8604.

[154] H. Wincencjusz, C.F. Yocum, H.J. van Gorkom, Activating anions that replace chloride in the oxygen-evolving complex of Photosystem II slow the kinetics of the terminal step in water oxidation and destabilize the $S_{2}$ and $S_{3}$ states, Biochemistry 38 (1999) 3719-3725.

[155] D.F. Ghanotakis, J.N. Topper, C.F. Yocum, Structural organization of the oxidizing side of Photosystem II: exogenous reductants reduce and destroy the Mncomplex in Photosystem II membranes depleted of the 17 and $23 \mathrm{kDa}$ polypeptides, Biochim. Biophys. Acta 767 (1984) 524-531.

[156] X. Yi, H. Liu, S.R. Hargett, L.K. Frankel, T.M. Bricker, The PsbP protein is required for Photosystem II complex assembly/stability and photoautotrophy in Arabidopsis thaliana, J. Biol. Chem. 34 (2007) 24833-24841.

[157] J.L. Roose, L.K. Frankel, T.M. Bricker, Documentation of significant electron transport defects on the reducing side of Photosystem II upon removal of the PsbP and PsbQ extrinsic proteins, Biochemistry 49 (2010) 36-41.

[158] X. Yi, S.R. Hargett, L.K. Frankel, T.M. Bricker, The PsbQ protein is required in Arabidopsis for Photosystem II assembly/stability and photoautotrophy under low light conditions, J. Biol. Chem. 281 (2006) 26260-26267.

[159] M. Tomita, K. Ifuku, F. Satoh, T. Noguchi, FTIR evidence that the PsbP extrinsic protein induces protein conformational changes around the oxygen-evolving Mn cluster in Photosystem II, Biochemistry 48 (2009) 6318-6325.

[160] T. Ono, S. Izawa, Y. Inoue, Structural and functional modulation of the manganese cluster in $\mathrm{Ca}^{+2}$-depleted Photosystem II by binding of the $24 \mathrm{kDa}$ extrinsic protein, Biochemistry 31 (1992) 7648-7655.

[161] K. Ifuku, T. Nakatsu, R. Shimamoto, Y. Yamamoto, S. Ishihara, H. Kato, F. Sato, Structure and function of the PsbP protein of Photosystem II from higher plants, Photosynth. Res. 84 (2005) 251-255.

[162] K. Ifuku, F. Sato, Importance of the N-terminal sequence of the extrinsic $23 \mathrm{kDa}$ polypeptide in Photosystem II in ion retention in oxygen evolution, Biochim. Biophys. Acta 1546 (2001) 196-204.

[163] A. Villarejo, T. Shutova, O. Moskvin, M. Forssen, V.V. Klimov, G. Samuelsson, A Photosystem II-associated carbonic anhydrase regulates the efficiency of photosynthetic oxygen evolution, EMBO J. 21 (2002) 1930-1938.

[164] A.V. Shitov, O.V. Pobeguts, T.N. Smolova, S.I. Allakhverdiev, V.V. Klimov, Manganese-dependent carboanhydrase activity of Photosystem II proteins, Biochemistry (Mosc.) 74 (2009) 509-517.

[165] B.C. Tripathy, P. Mohanty, Zinc-inhibited electron transport of photosynthesis in isolated barley chloroplasts, Plant Physiol. 66 (1980) 1174-1178.

[166] R.M. Chaloub, C.C.P. de Magalhães, C.P. dos Santos, Early toxic effects of zinc on PSII of Synechococcus aquatilis F. aquatilis (Cyanophyceae), J. Phycol. 41 (2005) 1162-1168. 
167] S. Reiland, G. Messerli, K. Baerenfaller, B. Gerrits, A. Endler, J. Grossmann, W. Gruissem, S. Baginsky, Large-scale Arabidopsis phosphoproteome profiling reveals novel chloroplast kinase substrates and phosphorylation networks, Plant Physiol. 150 (2009) 889-903.

[168] K. Lohrig, B. Müller, J. Davydova, D. Leister, D.A. Wolters, Phosphorylation site mapping of soluble proteins: bioinformatical filtering reveals potential plastidic phosphoproteins in Arabidopsis thaliana, Planta 229 (2009) 1123-1134.

[169] K. Ifuku, J. Yamamoto, T.-a. Ono, S. Ishihara, F. Sato, PsbP protein, but not PsbQ protein, is essential for the regulation and stabilization of Photosystem II in higher plants, Plant Physiol. 139 (2005) 1175-1184.

[170] K. Ido, K. Ifuku, Y. Yamamoto, S. Ishihara, A. Murakami, K. Takabe, C. Miyake, F. Sato, Knockdown of PsbP protein does not prevent assembly of the dimeric PSII core complex but impairs accumulation of Photosystem II supercomplexes in tobacco, Biochim. Biophys. Acta 1787 (2009) 873-881.

[171] X. Yi, S. Hargett, L.K. Frankel, T.M. Bricker, The PsbP protein, but not the PsbQ protein, is required for normal thylakoid membrane architecture in Arabidopsis thaliana, FEBS Lett. 583 (2009) 2142-2147.

[172] W.F. Ettinger, S.M. Theg, Physiologically active chloroplasts contain pools of unassembled extrinsic proteins of the photosynthetic oxygen-evolving enzyme complex in the thylakoid lumen, J. Cell Biol. 115 (1991) 321-328.

[173] A. Hashimoto, W.F. Ettinger, Y. Yamamoto, S.M. Theg, Assembly of newly imported oxygen-evolving complex subunits in isolated chloroplasts: sites of assembly and binding mechanism, Plant Cell 9 (1997) 441-452.

[174] C. de Vitry, J. Olive, D. Drapier, M. Recouvreur, F.A. Wollman, Post-translationa events leading to the assembly of Photosystem II protein complex a study using photosynthesis mutants from Chlamydomonas reinhardtii, J. Cell Biol. 109 (1989) 991-1006.

[175] S.P. Mayfield, M. Schirmer-Rahire, G.Z. Frandk, J.D. Rochaix, Analysis of the genes of the OEE1 and OEE3 proteins of the Photosystem II complex from Chlamydomonas reinhardtii, Plant Mol. Biol. 12 (1989) 683-693.

[176] U. Ljungberg, H.-E. Akerlund, C. Larsson, B. Andersson, Identification of polypeptides associated with the 23 and $33 \mathrm{kDa}$ proteins of photosynthetic oxygen evolution, Biochim. Biophys. Acta 767 (1984) 145-152.

[177] A. Webber, L.C. Packman, J.C. Gray, A $10 \mathrm{kDa}$ polypeptide associated with the oxygen-evolving complex of Photosystem II has a putative C-terminal noncleavable thylakoid transfer domain, FEBS Lett. 242 (1989) 435-438.

[178] U. Ljungberg, H.E. Åkerlund, B. Andersson, Isolation and characterization of the $10-\mathrm{kDa}$ and 22-kDa polypeptides of higher-plant Photosystem 2, Eur. J. Biochem. 158 (1986) 477-478.

[179] R. Harrer, R. Bassi, M.G. Testi, C. Schafer, Nearest-neighbor analysis of a Photosystem II complex from Marchantia polymorpha L. (liverwort), which contains reaction center and antenna proteins, Eur. J. Biochem. 255 (1998) 196-205.

[180] M. Suorsa, S. Sirpio, Y. Allahverdiyeva, V. Paakkarinen, F. Mamedov, S. Styring, E. Aro, PsbR, a missing link in the assembly of the oxygen-evolving complex of plant Photosystem II, J. Biol. Chem. 281 (2006) 145-150.

[181] M. Hager, M. Hermann, K. Biehler, A. Krieger-Liszkay, R. Bock, Lack of the small plastid-encoded PsbJ polypeptide results in a defective water-splitting apparatus of Photosystem II, reduced photosystem I levels, and hypersensitivity to light J. Biol. Chem. 277 (2002) 14031-14039.

[182] M. Suorsa, R.E. Regel, V. Paakkarinen, N. Battchikova, R.G. Herrmann, E.-M. Aro Protein assembly of Photosystem II and accumulation of subcomplexes in the absence of low molecular mass subunits PsbL and PsbJ, Eur. J. Biochem. 271 (2003) 96-107.

[183] Y.B.B. Zhang, I-TASSER server for protein 3D structure prediction, BMC Bioinformatics 9 (2008) 40-48.

[184] A. Roy, A. Kucukural, Y. Zhang, I-TASSER: a unified platform for automated protein structure and function prediction, Nat. Protoc. 5 (2010) 725-738.

[185] J. Stockhaus, M. Hofer, G. Renger, P. Westhoff, T. Wydrzynski, L. Willmitzer, Antisense RNA efficiently inhibits formation of the $10 \mathrm{kD}$ polypeptide of Photosystem II in transgenic potato plants: analysis of the role of the $10 \mathrm{kD}$ protein, EMBO J. 9 (1990) 3013-3021.

[186] Y. Allahverdiyeva, F. Mamedov, M. Suorsa, S. Styring, I. Vass, E.-M. Aro, Insights into the function of PsbR protein in Arabidopsis thaliana, Biochim. Biophys. Acta 1767 (2007) 677-685.

[187] H. Liu, L.K. Frankel, T.M. Bricker, Characterization and complementation of a psbR mutant in Arabidopsis thaliana, Arch. Biochem. Biophys. 489 (2009) 34-40.

[188] M.V. Turkina, J. Kargul, A. Blanco-Rivero, A. Villarejo, J.B. Barber, A.V. Vener, Environmentally modulated phosphoproteome of photosynthetic membranes in the green alga Chlamydomonas reinhardtii, Mol. Cell. Proteomics 5 (2006) $1412-1425$.

[189] N. Sugiyama, H. Nakagami, K. Mochida, A. Daudi, M. Tomita, K. Shirasu, Y. Ishihama, Large-scale phosphorylation mapping reveals the extent of tyrosine phosphorylation in Arabidopsis, Mol. Syst. Biol. 4 (2008) 193.

[190] P. Pesaresi, M. Pribil, T. Wunder, D. Leister, Dynamics of reversible protein phosphorylation in thylakoids of flowering plants: the roles of STN7, STN8 and TAP38, Biochim. Biophys. Acta 1807 (2011) 887-896.

[191] N. Kamiya, J.-R. Shen, Crystal structure of oxygen-evolving Photosystem II from Thermosynechococcus vulcanus at $3.7 \AA ̊$ resolution, Proc. Natl. Acad. Sci. U.S.A. 100 (2003) 98-103.

[192] A.C. Stewart, U. Ljungberg, H.-E. Akerlund, B. Anderson, Studies on the polypeptide composition of the cyanobacterial oxygen-evolving complex, Biochim. Biophys. Acta 808 (1985) 353-362.

[193] J.-R. Shen, M. Ikeuchi, Y. Inoue, Stoichiometric association of extrinsic cytochrome $c_{550}$ and $12 \mathrm{kDa}$ protein with a highly purified oxygen evolving
Photosystem II core complex from Synechococcus vulcanus, FEBS Lett. 301 (1992) 145-149.

[194] I. Enami, H. Murayama, H. Ohta, M. Kamo, K. Nakazato, J.R. Shen, Isolation and characterization of a Photosystem II complex from the red alga Cyanidium caldarium: association of cytochrome $\mathrm{C}_{550}$ and a $12 \mathrm{kDa}$ protein with the complex, Biochim. Biophys. Acta 1232 (1995) 208-216.

[195] H. Ohta, A. Okumura, S. Okuyama, A. Akiyama, M. Iwai, S. Yoshihara, J.-R. Shen, M. Kamo, I. Enami, Cloning, expression of the $p s b U$ gene, and functional studies of the recombinant 12-kDa protein of Photosystem II from a red alga Cyanidium caldarium, Biochem. Biophys. Res. Commun. 260 (1999) 245-250.

[196] I. Enami, T. Suzuki, O. Tada, Y. Nakada, K. Nakamura, A. Tohri, H. Ohta, I. Inoue, J.-R. Shen, Distribution of the extrinsic proteins as a potential marker for the evolution of photosynthetic oxygen-evolving Photosystem II, FEBS J. 272 (2005) 5020-5030.

[197] R. Nagao, A. Moriguchi, T. Tomo, A. Niikura, S. Nakajima, T. Suzuki, A. Okumura, M. Iwai, J.-R. Shen, M. Ikeuchi, I. Enami, Binding and functional properties of five extrinsic proteins in oxygen-evolving Photosystem II from a marine centric diatom, Chaetoceros gracilis, J. Biol. Chem. 258 (2010) 29191-29199.

[198] B. Thangaraj, C.M. Ryan, P. Souda, K. Krause, K.F. Faull, A.P.M. Weber, P. Fromme, J.P. Whitelegge, Data-directed top-down Fourier-transform mass spectrometry of a large integral membrane protein complex: Photosystem II from Galdieria sulphuraria, Proteomics 10 (2010) 3644-3656

[199] J.-R. Shen, Y. Inoue, Binding and functional properties of two new extrinsic components, cytochrome $\mathrm{c}_{550}$ and a $12-\mathrm{kDa}$ protein, in cyanobacterial Photosystem II, Biochemistry 32 (1993) 1825-1832.

[200] J.-R. Shen, M. Ikeuchi, Y. Inoue, Analysis of the psbU gene encoding the 12-kDa extrinsic protein of Photosystem II and studies on its role by deletion mutagenesis in Synechocystis sp. PCC 6803, J. Biol. Chem. 272 (1997) $17821-17826$.

[201] J.J. Eaton-Rye, J.A. Shand, W.S. Nicoll, pH-dependent photoautotrophic growth of specific Photosystem II mutants lacking lumenal extrinsic polypeptides in Synechocystis sp. PCC 6803, FEBS Lett. 543 (2003) 148-153.

[202] T.C. Summerfield, J.J. Eaton-Rye, L.A. Sherman, Global gene expression of a $\triangle$ PsbO: $\triangle$ PsbU mutant and a spontaneous revertant in the cyanobacterium Synechocystis sp. PCC 6803, Photosynth. Res. 94 (2007) 265-274.

[203] S. Clarke, J. Eaton-Rye, Mutation of 363-Phe in the Photosystem II protein CP47 impairs photoautotrophic growth, alters the chloride requirement, and prevents photosynthesis in the absence of either PSII-O or PSII-V in Synechocystis sp. PCC 6803, Biochemistry 38 (1999) 2707-2715.

[204] N. Inoue-Kashino, Y. Kashino, K. Satoh, I. Terashima, H.B. Pakrasi, PsbU provides a stable architecture for the oxygen-evolving system in cyanobacterial Photosystem II, Biochemistry 44 (2005) 12214-12228.

[205] J.-R. Shen, M. Qian, Y. Inoue, R.L. Burnap, Functional characterization of Synechocystis sp. PCC $6803 \Delta$ psbU and $\Delta$ psbV mutants reveals important roles of cytochrome c-550 in cyanobacterial oxygen evolution, Biochemistry 37 (1998) 1551-1558.

[206] A. Kimura, J.J. Eaton-Rye, E.H. Morita, Y. Nishiyama, H. Hayashi, Protection of the oxygen-evolving machinery by the extrinsic proteins of Photosystem II is essential for development of cellular thermotolerance in Synechocystis sp. PCC 6803, Plant Cell Physiol. 43 (2002) 932-938.

[207] J. Veerman, F.K. Bentley, J.J. Eaton-Rye, C.W. Mullineaux, S. Vasil'ev, D. Bruce, The PsbU subunit of Photosystem II stabilizes energy transfer and primary photochemistry in the phycobilisome-Photosystem II assembly of Synechocystis sp. PCC 6803, Biochemistry 44 (2005) 16939-16948.

[208] I. Balint, J. Bhattacharya, A. Perelman, D. Schatz, Y. Moskovitz, N. Keren, R. Schwarz, Inactivation of the extrinsic subunit of Photosystem II, PsbU, in Synechococcus PCC 7942 results in elevated resistance to oxidative stress, FEBS Lett. 580 (2006) 2117-2122.

[209] P. Mohanty, V.D. Kreslavski, D.A. Los, V.V. Klimov, M. Mimuro, R. Carpentier, S.I. Allakhverdiev, Heat stress: susceptibility, recovery and regulation, in: J.J. EatonRye, B.C. Tripathy, T.D. Sharkey (Eds.), Photosynthesis: Plastid Biology, Energy Conversion and Plastid Biology, Advances in Photosynthesis and Respiration, vol. 34, Springer, Dordrecht, in press.

[210] Y. Nishiyama, H. Hayashi, T. Watanabe, N. Murata, Photosynthetic oxygen evolution is stabilized by cytochrome $c_{550}$ against heat inactivation in Synechococcus sp. PCC 7002, Plant Physiol. 105 (1994) 1313-1319.

[211] Y. Nishiyama, D.A. Los, H. Hayashi, N. Murata, Thermal protection of the oxygen evolving machinery by PsbU, an extrinsic protein of Photosystem II, in Synechococcus species PCC 7002, Plant Physiol. 115 (1997) 1473-1480.

[212] Y. Nishiyama, D.A. Los, N. Murata, PsbU, a protein associated with Photosystem II, is required for the acquisition of cellular thermotolerance in Synechococcus species PCC 7002, Plant Physiol. 120 (1999) 301-308.

[213] C.F. Yocum, The calcium and chloride requirements for the $\mathrm{O}_{2}$ evolving complex, Coord. Chem. Rev. 252 (2008) 296-305.

[214] A. Okumura, H. Ohta, Y. Inoue, I. Enami, Identification of functional domains of the extrinsic $12 \mathrm{kDa}$ protein in red algal PSII by limited proteolysis and directed mutagenesis, Plant Cell Physiol. 42 (2001) 1331-1337.

[215] A. Okumura, M. Sano, T. Suzuki, H. Tanaka, R. Nagao, K. Nakazato, M. Iwai, H. Adachi, J.-R. Shen, I. Enami, Aromatic structure of tyrosine-92 in the extrinsic PsbU protein of red algal Photosystem II is important for its functioning, FEBS Lett. 581 (2007) 5255-5258.

[216] N. Knoepfle, T.M. Bricker, C. Putnam-Evans, Site-directed mutagenesis of basic arginine residues 305 and 342 in the CP 43 protein of Photosystem II affects oxygen-evolving activity in Synechocystis 6803, Biochemistry 38 (1999) $1582-1588$. 
[217] A. Young, M. McChargue, T.M. Bricker, L.K. Frankel, C. Putnam-Evans, Alterations of the oxygen-evolving apparatus in an ${ }^{305} \mathrm{Arg}$ to ${ }^{305} \mathrm{~S}$ mutant in the $\mathrm{CP} 43$ protein of Photosystem II from Synechocystis sp. PCC 6803 under chloride-limiting conditions, Biochemistry 41 (2002) 15747-15753.

[218] T.M. Bricker, A. Young, L.K. Frankel, C. Putnam-Evans, Introduction of the ${ }^{305} \mathrm{Arg} \rightarrow{ }^{305}$ Ser mutation in the large extrinsic loop E of the CP43 protein of Synechocystis sp. PCC 6803 leads to the loss of cytochrome $c_{550}$ binding to Photosystem II, Biochim. Biophys. Acta 1556 (2002) 92-96.

[219] I. Enami, S. Yoshihara, A. Tohri, A. Okumura, H. Ohta, J.R. Shen, Cross-reconstitution of various extrinsic proteins and Photosystem II complexes from cyanobacteria, red algae and higher plants, Plant Cell Physiol. 41 (2000) 1354-1364.

[220] I. Enami, M. Iwai, A. Akiyama, T. Suzuki, A. Okumura, T. Katoh, O. Tada, H. Ohta, J.-R Shen, Comparison of binding and functional properties of two extrinsic components, cyt c550 and a $12 \mathrm{kDa}$ protein, in cyanobacterial PSII with those in red algal PSII, Plant Cell Physiol. 44 (2003) 820-827.

[221] R.W. Holton, J. Myers, Cytochromes of a blue-green alga: extraction of a c-type with a strongly negative redox potential, Science 142 (1963) 234-235.

[222] J.-R. Shen, M. Ikeuchi, Y. Inoue, Stoichiometric association of extrinsic cytochrome $\mathrm{c}_{550}$ and the $12 \mathrm{kDa}$ protein with a highly purified oxygen-evolving PS II core complex from Synechococcus vulcanus, FEBS Lett. 301 (1992) 145-149.

[223] C. Frazao, F.J. Enguita, R. Coelho, G.M. Sheldrick, J.A. Navarro, M. Hervas, M.A. De la Rosa, M.A. Carrondo, Crystal structure of low-potential cytochrome $\mathrm{c}_{549}$ from Synechocystis sp. PCC 6803 at 1.21 Å resolution, J. Biol. Inorg. Chem. 6 (2001) 324-332.

[224] M.R. Sawaya, D.W. Krogmann, A. Serag, K.K. Ho, T.O. Yeates, C.A. Kerfeld, Structures of cytochrome $c_{549}$ and cytochrome $c_{6}$ from the cyanobacterium Arthrospira maxima, Biochemistry 40 (2001) 9215-9225.

[225] C.A. Kerfeld, M.R. Sawaya, H. Bottin, K.T. Tran, M. Sugiura, D. Cascio, A. Desbois, T.O. Yeates, D. Kirilovsky, A. Boussac, Structural and EPR characterization of the soluble form of cytochrome $c_{550}$ and of the $p s b V 2$ gene product from the cyanobacterium Thermosynechococcus elongatus, Plant Cell Physiol. 44 (2003) 697-706.

[226] L. Bumba, H. Havelkova-Dousova, M. Husak, F. Vacha, Structural characterization of Photosystem II complex from red alga Porphyridium cruentum retaining extrinsic subunits of the oxygen-evolving complex, Eur. J. Biochem. 271 (2004) 2967-2975.

[227] J.-R. Shen, R.L. Burnap, Y. Inoue, An independent role of cytochrome c-550 in cyanobacterial Photosystem II as revealed by double-deletion mutagenesis of the psbO and psbV genes in Synechocystis sp. PCC 6803, Biochemistry 34 (1995) 12661-12668.

[228] T. Morgan, J. Shand, S. Clarke, J. Eaton-Rye, Specific requirements for cytochrome c-550 and the manganese-stabilizing protein in photoautotrophic strains of Synechocystis sp. PCC 6803 with mutations in the domain ${ }^{351}$ Gly to ${ }^{436} \mathrm{Thr}$ of the chlorophyll binding protein CP47, Biochemistry 37 (1998) 14437-14449.

[229] H. Katoh, S. Itoh, J.R. Shen, M. Ikeuchi, Functional analysis of $p s b V$ and a novel ctype cytochrome gene $p s b \mathrm{~V} 2$ of the thermophilic cyanobacterium Thermosynechococcus elongatus Strain BP-1, Plant Cell Physiol. 42 (2001) 599-607.

[230] D. Kirilovsky, M. Roncel, A. Boussac, A. Wilson, J.L. Zurita, J.M. Ducruet, H. Bottin, M. Sugiura, J.M. Ortega, A.W. Rutherford, Cytochrome $c_{550}$ in the cyanobacterium Thermosynechococcus elongatus: study of redox mutants, J. Biol. Chem. 279 (2004) 52869-52880.

[231] H. Katoh, N. Hagino, A.R. Grossman, T. Ogawa, Genes essential to iron transport in the cyanobacterium Synechocystis sp. strain PCC 6803, J. Bacteriol. 183 (2001) 2779-2784.

[232] G.W. Pettigrew, G.R. Moore, Cytochromes c: Biological Aspects, Springer, Berlin Heidelberg New York, 1987.

[233] J. Alam, J. Sprinkle, M.A. Hermodson, D.W. Krogmann, Characterization of cytochrome c-550 from cyanobacteria, Biochim. Biophys. Acta 766 (1984) 317-321.

[234] C.W. Hoganson, G. Lagenfelt, L.-E. Andréasson, EPR and redox potentiometric studies of cytochrome c-549 of Anacystis nidulans, Biochim. Biophys. Acta 1016 (1990) 203-206.

[235] P.F. Kienzl, G.A. Peschek, Cytochrome c-549 - an endogenous cofactor of cyclic photophosphorylation in the cyanobacterium Anacystis nidulans? FEBS Lett. 162 (1983) 76-80.

[236] D.W. Krogmann, S. Smith, Low potential cytochrome $c_{550}$ function in cyanobacteria and algae, Curr. Res. Photosynth. 2 (1990) 687-690.

[237] L.Z. Morand, R.H. Cheng, D.W. Krogmann, K.K. Ho, Soluble electron transfer catalysts of cyanobacteria, in: D.A. Bryant (Ed.), The Molecular Biology of
Cyanobacteria, Advances in Photosynthesis, vol. 1, Kluwer Academic Publishers, Dordrecht, 1994, pp. 381-407.

[238] J.S. Vrettos, M.J. Reifler, O. Kievet, K.V. Lakshmi, J.C. de Paula, G.W. Brudvig, Factors that determine the unusually low reduction potential of cytochrome $c_{550}$ in cyanobacterial Photosystem II, J. Biol. Inorg. Chem. 6 (2001) 708-716.

[239] M. Roncel, A. Boussac, J.L. Zurita, H. Bottin, M. Sugiura, D. Kirilovsky, J.M. Ortega Redox properties of the Photosystem II cytochromes $b_{559}$ and $c_{550}$ in the cyanobacterium Thermosynechococcus elongatus, J. Biol. Inorg. Chem. 8 (2003) 206-216.

[240] H. Ishikita, E.W. Knapp, Redox potential of cytochrome $c_{550}$ in the cyanobacterium Thermosynechococcus elongatus, FEBS Lett. 579 (2005) 3190-3194.

[241] H. Andrews, Z. Li, A. Altuve-Blanco, M. Rivera, R.L. Burnap, Expression, mutagenesis, and characterization of recombinant low-potential cytochrome c550 of Photosystem II, Biochemistry 44 (2005) 6092-6100.

[242] F. Guerrero, A. Sedoud, D. Kirilovsky, A.W. Rutherford, J.M. Ortega, M. Roncel, A high redox potential form of cytochrome $c_{550}$ in Photosystem II from Thermosynechococcus elongatus, J. Biol. Chem. 286 (2011) 5985-5994.

[243] Y. Kashino, W.M. Lauber, J.A. Carroll, Q. Wang, J. Whitmarsh, K. Satoh, H.B. Pakrasi, Proteomic analysis of a highly active Photosystem II preparation from the cyanobacterium Synechocystis sp. PCC 6803 reveals the presence of novel polypeptides, Biochemistry 41 (2002) 8004-8012.

[244] T. Ujihara, I. Sakurai, N. Mizusawa, H. Wada, A method for analyzing lipidmodified proteins with mass spectrometry, Anal. Biochem. 374 (2008) 429-431.

[245] R.D. Fagerlund, J.J. Eaton-Rye, The lipoproteins of cyanobacterial Photosystem II, J. Photochem. Photobiol. B: Biol. 104 (2011) 191-203.

[246] T.M. Bricker, J. Morvant, N. Masri, H. Sutton, L.K. Frankel, Isolation of a highly active Photosystem II preparation from Synechocystis 6803 using a histidinetagged mutant of CP 47, Biochim. Biophys. Acta 1409 (1998) 50-57.

247] M.M. Nowaczyk, R. Hebeler, E. Schlodder, H.E. Meyer, B. Warscheid, M. Rogner, Psb27, a cyanobacterial lipoprotein, is involved in the repair cycle of Photosystem II, Plant Cell 18 (2006) 3121-3131.

[248] P.J. Nixon, F. Michoux, J. Yu, M. Boehm, J. Komenda, Recent advances in understanding the assembly and repair of Photosystem II, Ann. Bot. 106 (2010) $1-16$.

[249] J.J. Eaton-Rye, Requirements for different combinations of the extrinsic proteins in specific cyanobacterial Photosystem II mutants, Photosynth. Res. 84 (2005) 275-281.

[250] J. De Las Rivas, M. Baklsera, J. Barber, Evolution of oxygenic photosynthesis: genome wide analysis of the OEC proteins, Trends Plant Sci. 9 (2004) 18-25.

[251] N. Sato, Phylogenomic and structural modeling analyses of the PsbP superfamily reveal multiple small segment additions in the evolution of Photosystem IIassociated PsbP protein in green plants, Mol. Phylogenet. Evol. 56 (2010) 176-186.

252] S. Ishihara, A. Takabayashi, K. Ido, T. Endo, K. Ifuku, F. Sato, Distinct functions for the two PsbP-like proteins PPL1 and PPL2 in the chloroplast lumen of Arabidopsis, Plant Physiol. 145 (2007) 668-679.

[253] N. Bondarava, A. Krieger-Liszkay, Manganese binding to the $23 \mathrm{kDa}$ extrinsic protein of Photosystem II, Biochim. Biophys. Acta 1767 (2007) 583-588.

[254] E. Haag, J.J. Eaton-Rye, G. Renger, W.F.J. Vermaas, Functionally important domains of the large hydrophilic loop of $\mathrm{CP} 47$ as probed by oligonucleotidedirected mutagenesis in Synechocystis sp. PCC 6803, Biochemistry 32 (1993) 4444-4454.

[255] P. Beneš, E. Chovancová, B. Kozlíková, A. Pavelka, O. Strnad, J. Brezovský, V. Šustr, M. Klvaňa, T. Szabó, A. Gora, M. Zamborský, L. Biedermannová, P. Medek, J. Damborský, J. Sochor, CAVER 2.1, Software (2010).

[256] T.C. Summerfield, R.T. Winter, J.J. Eaton-Rye, Investigation of a requirement for the PsbP-like protein in Synechocystis sp. PCC 6803, Photosynth. Res. 84 (2005) 263-268.

[257] J.-R. Shen, W. Vermaas, Y. Inoue, The role of cytochrome c-550 as studied through reverse genetics and mutant characterization in Synechocystis sp. PCC 6803, J. Biol. Chem. 270 (1995) 6901-6907.

[258] D. Sveshnikov, C. Funk, W.P. Schröder, The PsbP-like protein (sll1418) of Synechocystis sp. PCC 6803 stabilises the donor side of Photosystem II, Photosynth. Res. 93 (2007) 101-109.

[259] W.L. DeLano, The PyMOL molecular graphics system, Software (2002).

[260] M. Petřek, M. Otyepka, P. Banáš, P. Košinová, J. Koča, CAVER: a new tool to explore routes from protein clefts, pockets and cavities, BMC Bioinformatics 7 (2006) 316. 